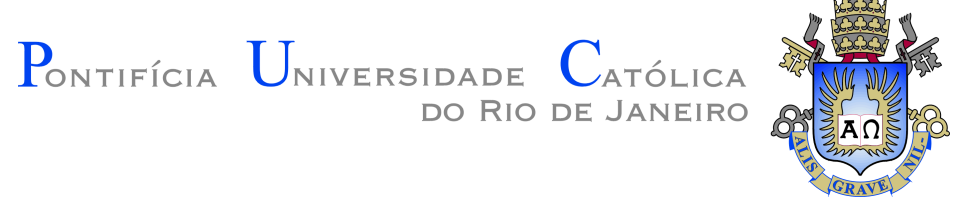

Jonatan de Oliveira Dias

\title{
Rock Physics Modeling Evaluation for \\ Carbonate Reservoirs
}

Thesis presented to the Programa de Pós-graduação em Engenharia Civil da PUC-Rio in partial fulfillment of the requirements for the degree of Mestre em Engenharia Civil .

Advisor : Prof. Raquel Quadros Velloso

Co-advisor: Dr. Cassiane Maria Ferreira Nunes 


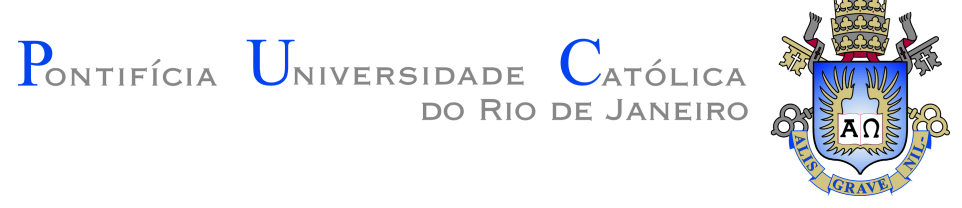

Jonatan de Oliveira Dias

\section{Rock Physics Modeling Evaluation for \\ Carbonate Reservoirs}

Thesis presented to the Programa de Pós-graduação em Engenharia Civil da PUC-Rio in partial fulfillment of the requirements for the degree of Mestre em Engenharia Civil. Approved by the undersigned Examination Committee.

Prof. Raquel Quadros Velloso

Advisor

Departamento de Engenharia Civil e Ambiental - PUC-Rio

Dr. Cassiane Maria Ferreira Nunes

Co-advisor

Geophysicist - Petrobras

Dr. Lucia Duarte Dillon

Former Senior Geophysicist - Petrobras

Dr. Flávia de Oliveira Lima Falcão

Petroleum Engineer - Petrobras

Prof. Eurípides do Amaral Vargas Junior Departamento de Engenharia Civil e Ambiental - PUC-Rio

Prof. Marcio da Silveira Carvalho

Coordenador Setorial do Centro Técnico Científico - PUC-Rio 
All rights reserved.

\section{Jonatan de Oliveira Dias}

I have a B.Sc. in Geophysics from Universidade Federal Fluminense (UFF-RJ) where I acquired experience with seismic modeling, seismic processing and basin analysis. During January/2016 until January/2018, I also worked as a research graduate intern in the IBM Research Lab/Brazil. In December/2017, I concluded my M.Sc. applying rock physics models in order to quantitatively understand the elastic attributes of carbonate rocks taking into consideration a stochastic perspective in a Bayesian Framework.

Bibliographic data

Dias, Jonatan de Oliveira

Rock Physics Modeling Evaluation for Carbonate Reservoirs / Jonatan de Oliveira Dias; advisor: Raquel Quadros Velloso; co-advisor: Cassiane Maria Ferreira Nunes. - Rio de janeiro: PUC-Rio, Departamento de Engenharia Civil e Ambiental , 2017.

v., 126 f: il. color. ; $30 \mathrm{~cm}$

Dissertação (mestrado) - Pontifícia Universidade Católica do Rio de Janeiro, Departamento de Engenharia Civil e Ambiental .

\section{Inclui bibliografia}

1. Engenharia Civil - Teses. 2. Física de Rochas;. 3. Carbonatos;. 4. Modelo de Xu \& Payne;. 5. Modelo TMatrix. I. Velloso, Raquel Quadros. II. Nunes, Cassiane Maria Ferreira. III. Pontifícia Universidade Católica do Rio de Janeiro. Departamento de Engenharia Civil e Ambiental . IV. Título. 


\section{Acknowledgments}

First of all, I would like to thank God for all the oportunities that $\mathrm{He}$ provides to me.

I would like to thank my wife Paula for all the comprehension, love and kindness.

I would like to thank all the patience and comprehension that my parents and relatives had during these two years of intense dedication to my academic and professional life.

I would like to thank Prof. Raquel who trusted and believed that I could make a good work during my Master's Degree and accepted to be my Advisor. It was a great pleasure work with you during these two years. Thank you for all the patience and kindness during the several meetings that we had to discuss my questions and issues about rock physics. Your intelligence and commitment are incredible, thank you very much for everything, I feel honored to had you as my Advisor.

I also would like to thank my Co-Advisor Cassiane. It is not easy to express how grateful I am for all the e-mails answered, all the meetings that we had, and you always had the patience and kindness to answer all my questions. It was a great pleasure to meet you and see that we have professionals so dedicated to their duties. You are an example and I feel myself very honored to learn about rock physics with you. Julio, it was also a great pleasure to meet you and learn about rock physics with you. Thank you for all the help and questions answered.

I also would like to thank Lucia Dillon for the time, support and patience. Thank you so much for the ideas, insights and questions answered. It was a great pleasure learn with you.

I also would like to make a special thank to Gregor Baechle, Anders Draege, Dario Grana and Tapan Mukerji who always made themselves available answering all my questions. It was incredible to learn with each one of you about rock physics. 
I would like to thank all the people that somehow contributed to my academic - professional life and helped me in order to conclude this dissertation.

I would like to thank Rita and Fátima for all the patience and kindness during these two years.

I would like to thank CAPES/CNPq and PUC-Rio for sponsoring this research. 


\section{Abstract}

Dias, Jonatan de Oliveira; Velloso, Raquel Quadros (Advisor); Nunes, Cassiane Maria Ferreira (Co-Advisor). Rock Physics Modeling Evaluation for Carbonate Reservoirs. Rio de Janeiro, 2017. 126p. Dissertação de mestrado - Departamento de Engenharia Civil e Ambiental, Pontifícia Universidade Católica do Rio de Janeiro.

Since the 80's, data-driven approaches have been used for fluids identification and reservoir characterization of siliciclastic and carbonate rocks mainly regarding seismic amplitudes analyses. However, techniques successfully applied for siliciclastic rocks, such as: AVO analysis, seismic inversions and DHI (Direct Hydrocarbon Indicators) ranking revealed not have achieved the same outstanding and reliable results for heterogeneous carbonate rocks. On the other hand, several articles demonstrate that reservoir characterization workflows with rock physics models embedded have been reaching a robust success in order to obtain petrophysical properties and elastic attributes of both rocks, from the seismic and well logs, in a model-driven approach focused on the reservoirs microstructural information. In this way, taking into account the importance of applying rock physics models in the scope of reservoir characterization, two rock physics models - Xu \& Payne and T-Matrix - were applied, compared and their parameters were stochastically evaluated and optimized in a Bayesian framework. Through this approach, it was possible to estimate, in a reliable manner, the elastic attributes of a carbonate reservoir (coquinas) taking into consideration different kinds of uncertainties. Furthermore, after the calibration in the well location and validation of both rock physics models for other wells, sensitivity analyses were conducted in order to quantitatively understand how the coquinas elastic attributes behave regarding the variations in the reservoir mineralogical content, pore shapes and fluids.

\section{Keywords}

Rock Physics; Carbonates; Xu \& Payne Model; T-Matrix Model 


\section{Resumo}

Dias, Jonatan de Oliveira; Velloso, Raquel Quadros; Nunes, Cassiane Maria Ferreira. Avaliação de Modelos de Física de Rochas para Reservatórios Carbonáticos. Rio de Janeiro, 2017. 126p. Dissertação de Mestrado - Departamento de Engenharia Civil e Ambiental, Pontifícia Universidade Católica do Rio de Janeiro.

Desde a década de 80, abordagens data-driven têm sido utilizadas para identificação de fluidos e caracterização de reservatórios carbonáticos e siliciclásticos principalmente em relação à análise das amplitudes sísmicas. No entanto, técnicas aplicadas com sucesso para rochas siliciclásticas, como por exemplo: Análise AVO, inversões sísmicas e IDH (Indicadores Diretos de Hidrocarbonetos) revelaram não obter o mesmo êxito para reservatórios carbonáticos heterogêneos. Em contrapartida, diversos artigos mostram que fluxos de caracterização de reservatórios com modelos de física de rochas incorporados têm alcançado grande sucesso para obtenção de propriedades petrofísicas e atributos elásticos de ambas as rochas, utilizando sísmicas e well logs, em uma abordagem model-driven, focada nas características microestruturais do reservatório. Dessa forma, levando em consideração a importância de se utilizar modelos de física de rochas no escopo da caracterização de reservatórios, dois modelos de física de rochas Xu \& Payne e T-Matrix - foram aplicados, comparados e seus parâmetros foram estocasticamente avaliados e otimizados em um arcabouço Bayesiano. Através dessa abordagem, foi possível estimar, de uma forma confiável, os atributos elásticos de um reservatório carbonático (coquinas) levando em consideração diversos tipos de incertezas. Além disso, após a calibração e validação de ambos os modelos de física de rochas para diferentes poços, análises de sensibilidade foram realizadas para compreensão de forma quantitativa do comportamento dos atributos elásticos das coquinas em relação às alterações do conteúdo mineralógico, tipos de poro e fluidos desse reservatório.

\section{Palavras-chave}

Física de Rochas; Carbonatos; Modelo de Xu \& Payne; Modelo T-Matrix 


\section{Table of contents}

1 Introduction $\quad 14$

2 Rock Physics of Carbonate Rocks 18

2.1 Main Factors which affect the Carbonate Rock Elastic Attributes 21

3 Rock Physics Models Review $\quad 27$

3.1 Xu and Payne Rock Physics Model 29

3.1.1 Effective Solid Elastic Media 33

3.1.1.1 Voigt and Reuss Bounds 33

3.1.1.2 Voigt - Reuss - Hill Average 34

3.1.2 Effective Moduli (Inclusion Methods) 34

3.1.2.1 Kuster and Toksoz Theory 34

3.1.2.2 Differential Effective Medium Theory (DEM) 39

3.1.3 Fluids 41

3.1.3.1 Wood's Mixing Law 41

3.1.3.2 Gassmann's Relations $\quad 42$

3.2 T-Matrix Rock Physics Model 43

3.2.1 T-Matrix Mathematical Formulations 45

$4 \quad$ Bayesian Inference Theory 49

4.1 Random Variables and Probability Density Functions - PDFs 50

4.2 Bayesian Inference $\quad 52$

4.3 Metrics to Measure the Models Accuracy 55

5 Methodology $\quad 57$

5.1 Dataset, Data Quality Control and Tools 57

5.1.1 Quality Control of Well Logs and Core Samples Measurements 57

5.1.2 Quality Control of the Thin Sections Images and Aspect Ratio Measurements $\quad 58$

5.1.3 Deploys of the Rock Physics Models and the DREAM Framework 59

5.2 Stochastic and Deterministic Parameters 60

5.2.1 Stochastic Parameters 61

5.2.1.1 Mineralogical Content - Bulk and Shear Elastic Moduli 61

5.2.1.2 Pores Aspect Ratios and their Volume Proportions 64

$\begin{array}{ll}\text { 5.2.2 Deterministic Parameters } & 70\end{array}$

$\begin{array}{lll}\text { 5.2.2.1 Fluids } & 70\end{array}$

5.2.2.2 Frequency, Relaxation Time Constant and Connectivity 72

6 Results and Discussions $\quad 74$

6.1 Rock Physics Parameters Evaluation Through the DREAM Algorithm 75

6.2 Rock Physics Modelings Validations and Comparisons - Well Logs 80

6.3 Rock Physics Modeling Validations and Comparisons - Core Samples 91

$\begin{array}{ll}\text { 6.4 Sensitivity Analyses } & 97\end{array}$

6.4.1 Sensitivity Analyses - Pores Aspect Ratios 98 
$\begin{array}{lll}\text { 6.4.2 Sensitivity Analyses - Fluids } & 103\end{array}$

6.4.3 Sensitivity Analyses - Mineralogical Content 106

7 Conclusions, Final Remarks and Future Work $\quad 110$

$\begin{array}{lll}7.1 & \text { Future Work } & 115\end{array}$

$\begin{array}{ll}\text { Bibliography } & 116\end{array}$

A Pores Aspect Ratio Measurements in the Thin Sections Images $\mathbf{1 2 5}$

B DREAM's outcomes - Xu and Payne and T-Matrix Rock Physics Models

- Well $49 \quad 126$ 


\section{List of figures}

Figure 2.1 Qualitative relation between fluids, petrophysical and rock properties with acoustic velocities. Image from Mavko et al., (2009) (68).

Figure 2.2 Crossplot $V_{p}-\phi$ for different carbonate core samples. Image from Eberli et al., (2003) (37).

Figure 2.3 Carbonate rocks thin sections illustrating different pore types and their respective "ideal" representations. Image from $\mathrm{Xu}$ and Payne (2009) (86).

Figure 2.4 (a) oolithic carbonate sand with interparticle porosity and (b) diagenetic process of dissolution generating moldic porosity in the carbonate rock. Image from Eberli et al., (2003) (37).

Figure 2.5 Moldic porosity generated through the diagenetic process of dissolution in both thin sections (mudstone and grainstone). Image from Bizotto (2014) (15).

Figure 2.6 Distribution of pore types in crossplot $V_{p}-\phi$ in the rock physics template of $\mathrm{Xu}$ and Payne. Image from $\mathrm{Xu}$ and Payne (2009) (86).

Figure 3.1 Diagram of the three steps of de Xu \& Payne rock physics model. Image from Xu and Payne (2009) (86).

Figure 5.1 Some of the thin sections images used to make the pores aspect ratios measurements where the blue color represents the pore space. Image from Bizotto (2014) (15).

Figure 5.2 Gaussian distribution representing the calcite elastic bulk modulus.

Figure 5.3 Gaussian distribution representing the calcite elastic shear modulus.

Figure 5.4 Histogram of all aspect ratio measurements in the 22 thin sections normalized by the volume percentage.

Figure 5.5 Gaussian Distribution Representing the Volume of Crack Pores.

Figure 5.6 Gaussian distribution representing the values of aspect ratio of the crack pores.

Figure 5.7 Gaussian distribution representing the values of aspect ratio measured in the thin sections.

Figure 6.1 Intervals of 95\% uncertainty regarding the parameters (black region) and total uncertainty (gray region) using the TMatrix Rock Physics Model - Well 49.

Figure 6.2 Histograms of the marginal posterior distributions of the parameters $V_{1}, \alpha_{1}, \alpha_{2}, \mathrm{~K}$ and $\mathrm{G}$ (Well 49) - T-Matrix Model. The blue crosses represent the MAP values. 
Figure 6.3 Marginal distributions (blue histograms) and bivariate scatter plots regarding the correlation between the parameters of the posterior distributions outputted through the DREAM algorithm (Well 49) - T-Matrix Model.

Figure 6.4 Rock Physics Models of Xu and Payne and T-Matrix settled over the crossplots $V_{p}-\phi$ of the Wells 36, 49 and 10 colored by the water saturation $\log \left(S_{w}\right)$.

Figure 6.5 Comparison between the elastic attributes estimations of both rock physics models for Well 49 and the effective porosity (PHIE) well log.

Figure 6.6 Rock physics models curves settled over the crossplot $V_{p}-\phi$ of Well 49 and error histograms regarding the difference between the modeled and the measured $\mathrm{P}$ wave velocities.

Figure 6.7 Comparison between the elastic attributes estimations of both rock physics models for Well 36 and the effective porosity (PHIE) well log.

Figure 6.8 Both rock physics models curves settled over the crossplot $V_{p}-\phi$ of Well 36 and error histograms regarding the difference between the modeled and the measured $\mathrm{P}$ wave velocities.

Figure 6.9 Comparison between the elastic attributes estimations of both rock physics models for Well 10 and the effective porosity (PHIE) well log.

Figure 6.10 Both rock physics models curves settled over the crossplot $V_{p}-\phi$ of Well 10 and error histograms regarding the difference between the modeled and the measured $\mathrm{P}$ wave velocities.

Figure 6.11 Saturated P wave velocities of the core samples following the trend of the three Wells 49, 36 and 10.

Figure 6.12 Comparison between the elastic attributes estimations of both rock physics models regarding the core samples and the models settled over the crossplot $V_{p}-\phi$.

Figure 6.13 Error histograms regarding the difference between the modeled and the measured $\mathrm{P}$ wave velocities for both models.

Figure 6.14 Comparison between the elastic attributes estimations of both rock physics models regarding the core samples and the models settled over the crossplot $V_{s}-\phi$.

Figure 6.15 Error histograms regarding the difference between the modeled and the measured $\mathrm{S}$ wave velocities for both models.

Figure 6.16 Comparison between the elastic attributes estimations of both rock physics models regarding the core samples and the models settled over the crossplot $V_{p} / V_{s}-\phi$.

Figure 6.17 Error histograms regarding the difference between the modeled and the measured $V_{p} / V_{s}$ ratio for both models.

Figure 6.18 Sensitivity analyses regarding different aspect ratios in the rock physics model of T-Matrix with $\alpha=0.016,0.01$ and 0.1 . 
Figure 6.19 Rock physics modelings regarding different aspect ratios using the rock physics model of T-Matrix with $\alpha=0.016,0.01$ and 0.1 over the crossplot $V_{p}-\phi$ of Well 36 .

Figure 6.20 Sensitivity analyses regarding different aspect ratios using the rock physics model of T-Matrix with $\alpha=0.016,0.1$, $0.2,0.4,0.6$ and 0.8 .

Figure 6.21 Rock physics modelings regarding different aspect ratios using the rock physics model of T-Matrix with $\alpha=0.016,0.1$, $0.2,0.4,0.6$ and 0.8 over the crossplot $V_{p}-\phi$ of Well 36 .

Figure 6.22 Sensitivity Analyses regarding fluids inclusions taking into consideration the hypotheses of all the pores fulfilled by brine (Track 2) and oil (Track 3).

Figure 6.23 Crossplot $V_{p}-\phi$ of the Well 36 colored by the water saturation $\log \left(S_{w}\right)$.

Figure 6.24 Sensitivity Analyses taking into consideration the hypotheses of all the pores fulfilled by brine or oil.

Figure 6.25 Simulations of the T-Matrix rock physics model representing the hypotheses of all the reservoir pores fulfilled by oil $\left(S_{w}=0\right)$ or water $\left(S_{w}=1\right)$ and the crossplot $V_{p}-\phi$ colored by the water saturation $\log \left(S_{w}\right)$.

Figure 6.26 Sensitivity analyses regarding different values of bulk and shear moduli using the T-Matrix rock physics model.

Figure A.1 Yellow ellipses fitted in the pores of the thin sections images through the ImageJ software. 


\section{List of tables}

Table 5.1 Values of Shear and Bulk moduli of Calcite and Dolomite Minerals.

Table 6.1 Means, Variances and Lower (LB) and Upper bounds

(UB) of the Prior Parameters Distributions

Table 6.2 DREAM's Outputs - Well 49 - T-Matrix Model 79

Table 6.3 DREAM's Outputs - Well 49 - Xu and Payne Model 79

Table 6.4 Metrics of Accuracy - Well 49 - P Wave Velocity 89

Table 6.5 Metrics of Accuracy - Well 36 - P Wave Velocity 89

Table 6.6 Metrics of Accuracy - Well 10 - P Wave Velocity 89

Table 6.7 Metrics of Accuracy - Core Samples - Xu \& Payne 96

Table 6.8 Metrics of Accuracy - Core Samples - T-Matrix 96

Table 6.9 Values of Shear and Bulk moduli of Calcite, Dolomite and Quartz Minerals.

Table 6.10 Bulk and Shear Moduli obtained using the Voigt-Reuss averages together with the Mixing Law of Hill and Density obtained through the Voigt's average.

Table B.1 RMSE of the MAP values using both Rock Physics models in the DREAM algorithm - Well 49. 


\section{1 \\ Introduction}

The oil \& gas industry is divided into three major sectors: upstream, midstream and downstream. The exploration and production phases are embedded in the upstream sector which is responsible for finding and extracting the oil and gas from the subsurface. Therefore, to reach successful results in the upstream, a clear understanding of the sedimentary basins is necessary through the analyses of the geological structures beneath the surface using geophysical methods. However, for exploration purposes, there is a task more important than delimiting the structural and stratigraphical regional frameworks of those basins through the analyses of 2D and 3D seismic data: The identification and characterization of possible reservoirs rocks through techniques which not only estimate but also relate the rocks petrophysical properties to their elastic attributes.

There are two main types of reservoirs rocks: siliciclastics and carbonates. The carbonate rocks have been reaching a high notoriety in recent years not only locally in Brazil's pre salt on the continental margin but also globally. In fact, approximately $50 \%$ of the worldwide production of oil and gas come from carbonate reservoirs in the sedimentary basins around the world. However, there are many distinctions between the siliciclastic and carbonate rocks in several aspects mainly regarding sedimentary facies, diagenetic processes and pore network (Choquette and Pray (1970) (25), Anselmetti and Eberli (1993) (), Anselmetti and Eberli (1999) (9) and Eberli et al., (2003) (37)). Carbonate rocks are highly affected by diagenetic processes - dissolution, cementation, compaction and others - which are responsible for changing the primary porosity - interparticle and intercrystaline Wang (1997) (90) generated during the rocks initial stages of diagenesis. In this way, due to the diagenetic processes, the different kinds of sedimentary facies, pore-type variety and others, some authors created classifications trying to ease the task of understanding those rocks frameworks. The following works have most relevance in the matter of carbonates classification: Folk (1959) (39), Folk (1962) (40), Dunham (1962) (34) and Embry and Klovan (1971) (38).

Lucia (2007) (62) stated that the main reason to conduct a reservoir characterization is to obtain three-dimensional images of petrophysical properties. 
However, the new discoveries about the complexity of carbonates framework led the academia and industry to develop new characterization techniques because the last ones used to extract important and reliable information of siliciclastic reservoirs were no longer trustworthy enough to characterize the required properties for carbonate rocks. Therefore, those techniques have been developed mainly because of the pore-type variety embedded in the carbonate rocks whereas siliciclastic rocks have a more homogeneous framework. In fact, some authors have been doing important studies in several aspects to better understand the relation between pore-type variety, petrophysical properties and elastic attributes of carbonate rocks, as follows: Agesborg (2007) (6), Agesborg et al., (2008) (7), Neto and Missagia (2012) (61), Kittridge (2015) (58), Kumar and Han (2005) (59), Anselmetti and Eberli (1997, 1993, 1999) $(1,, 9)$, Eberli et al., (2003) (37) and Baechle et al., (2004) (16).

The seismic reflection is the most widely used method in geophysics for oil and gas exploration. The measurements made in the seismic reflection are the waves travel times - between the source and the receivers - and the final outcome - after the application of seismic processing techniques - of this method is a stacked seismogram, also known as seismic or stacked seismic. Russel and Hampson (2006) (79) stated that initially, the seismic had the goal of only identifying geological structures beneath the surface. However, in the 70's, seismic amplitudes became very important because geophysicists found out that bright-spots could be a good DHI - Direct Hydrocarbon Indicator. Bright-spot is a fancy name for amplitudes anomalies which can be analyzed throughout the seismic. Nonetheless, those inferences through bright-spots in the seismic were very ambiguous and did not always lead to a successful indication of hydrocarbons as was expected (Chiburis et al., (1993) (24), Avseth et al., (2005) (12)).

AVO (Amplitude vs. Offset) analysis has been applied successfully as a geophysical technique since the 80's for a more reliable detection of amplitudes anomalies in the seismic, mainly, distinguishing gas-related amplitude anomalies and other types of anomalies for siliciclastic rocks (Ostrander (1984) (72)). Moreover, the academia and industry consider the AVO technique as a good DHI (Direct Hydrocarbon Indicator) - Chiburis et al., (1993) (24) and Mahmoud et al., (2008) (67) - and there is a great effort to develop applications of AVO technique for carbonates in recent years (Li et al., (2003), Isaac and Lawton (2003), Mahmoud et al., (2008) and Elbaz et al., (2010) (63, 52, 67, 36)). However, even the authors who work with AVO analysis are used to pointing out that the method has its limitations for carbonate rocks in the matter of reliable and confident inferences of fluids and lithological information of those 
heterogeneous rocks (Li et al., (2003) (63), Mahmoud et al., (2008) (67) and Isaac and Lawton (2009) (52)). As a matter of fact, AVO analysis is a technique embedded in the so-called data-driven approach as well as seismic inversions (acoustic and elastic) and other approaches related to machine learning scope, such as: neural networks, support vector machines and others (Kuroda et al., (2016) (57) and Cersosimo et al., (2016) (29)). A data-driven approach can be described as a technique for reservoir characterization which mostly takes the seismic information into consideration such as: amplitude, frequency, wavelength and other seismic attributes together with well logs or even only the seismic itself (pre stack or post stack).

As quickly as more data-driven techniques are being established, at least in recent years, a research field has been strongly developed and used inside of reservoir characterization workflows generating successful results for estimations of petrophysical properties and elastic attributes of siliciclastic and carbonate reservoirs from the seismic and well logs: Rock Physics (Avseth et al., (2005) (12), Draege (2012) (32), Sain et al., (2008) (81), Payne et al., (2010) (74), Ruiz and Dvorkin (2007) (78), Xu and Payne (2009) (86), Grana (2013, 2014) (46, 48), Jakobsen et al., (2003a,b) (54, 53), Spikes (2008) (82)). Reservoir characterization workflows with rock physics models embedded are classified as model-driven approaches (Coleou et al., (2006) (77)), because those workflows through those models not only relate the seismic to well log data in a "macro" perspective, but also to thin-sections, core samples and other valuable information - in a micro perspective - that improve the characterization of a reservoir with a physical reasoning behind the scenes through the link of the rock elastic attributes and its petrophysical properties.

Avseth et al., (2005) (12) stated that the goal of rock physics research is to understand and discover the seismic-to-reservoir relations. Rock physics models have the same meaning of petroelastic models, both of them are responsible for making the link between elastic attributes and petrophysical properties of a rock (Grana (2014), Allo et al., (2011) and Codo and Fournier (2016) $(48,14,28))$. Currently, those models have been used in several research lines achieving successful results not only in the matter of pore-type characterization (Zhao et al., (2013), Artola et al., (2016) (91, 10)), model-based seismic inversions (Coleou et al., (2006, 2012) and Allo et al., (2011) (77, 27, 14)) and 4D petrophysical seismic inversions (Coleou et al., (2013), Gjerding et al., (2010), Michou et al., (2013) and Machecler et al., (2009) (26, 47, 69, 70)) but as well as in reservoir engineering (Fahimudin (2009) and Rodrigues et al., (2012) (41, 80)). 
The petroelastic models are embedded with a set of equations - derived from the rock physics foundations - which are responsible for making the link between the petrophysical properties and the elastic attributes for both kind of rocks: siliciclastic and carbonates. Regarding carbonate rocks, the models try to simulate - in the most reliable possible way - those rocks heterogeneity. As it was already addressed previously, the carbonate rocks are highly affected by diagenetic processes which are responsible for changing the carbonates porenetwork in an abrupt way (Choquette and Pray (1970) (25)). Therefore, those processes can generate different pore types with several pore shapes as well as alterations in the carbonate matrix. In fact, the real challenge behind the rock physics modeling for carbonates is to represent with confidence the pore-type variety of these rocks through ellipses with different aspect ratios (taking into consideration an elliptical inclusion, the aspect ratio is the ratio between the pores minor and major axes of the ellipse).

This dissertation has two main goals. The first one is to address, compare and evaluate the rock physics models of Xu \& Payne and T-Matrix regarding elastic attributes estimations of a carbonate reservoir (coquinas). However, unfortunately, inserting different inputs inside of those models, similar outputs can be obtained. Therefore, in order to handle this non-uniqueness problem and reliably evaluate the outcomes of both models, their input parameters were stochastically settled and optimized in a Bayesian framework. In fact, the Bayesian inference has been a trend in many fields of research (Vrugt (2016) (84)). Specifically, in the rock physics scope, this approach provided the possibility to take into consideration the inherent uncertainties regarding the input parameters as well as the geological uncertainties. Thus, it was possible to achieve more accurate elastic attributes estimations and evaluation through the rock physics models despite their non-uniqueness issues. Secondly, after the parametrization and calibration in the well location, both rock physics models were extrapolated and validated for other wells and sensitivity analyses were conducted in order to quantitatively understand how the coquinas elastic attributes behave regarding the changes in the reservoir mineralogical content, pore shapes and fluids. 


\section{2}

\section{Rock Physics of Carbonate Rocks}

The real rocks found in nature are not composed by a homogeneous material or even a specific mineral. Rocks themselves are complex structures which are unique in several aspects of geology, sedimentology, diagenetic processes and others. However, try to understand those rocks is very important, because some of them can be reservoirs which can store billions of oil barrels. In fact, that is why rock physics models are relevant: To try to represent a reality which sometimes is too difficult to understand and make experiments with, in an attempt to extract valuable information and conclusions about those models responses. Despite the fact that it is not possible to take into consideration all the heterogeneities present in a reservoir, the models try to simplify what is really complicated through assumptions, approximations, and idealizations and simulate the rocks elastic attributes (Avseth et al., (2005) $(12))$.

One of the advantages of using rock physics models is to find a model which represents reliably the reservoir elastic attributes, hence, it is also possible to take advantage of the extrapolation power of these models to compute elastic attributes estimations far away from the well location, for instance. The process of identifying a rock physics model which fits and/or represents a reservoir or a dataset is called: rock physics diagnostics (Dvorkin et al., (2014) (30)), since the models estimate with confidence the reservoirs elastic attributes, the forward seismic modeling can be done and behaviors of seismic signatures can be observed changing the initial parameters of the model. The forward seismic modeling through rock physics models can be an important tool to reduce uncertainty. In fact, this is a worthy research field that is beyond the scope of this dissertation, although, the book: Seismic Reflections of Rock Properties of Dvorkin et al., (2014) (30) gives an extended review about this subject.

This whole introduction for this chapter is needed to give more insights of how rock physics models work and how they can be helpful to reduce uncertainty in the oil exploration process. The goal of rock physics models is to compute/calculate the rocks elastic attributes through inputs of those rocks petrophysical properties. However, several factors affect the rocks elastic 
attributes in different manners. Therefore, the main objective of this chapter is not to make a geological or even sedimentological overview about carbonate rocks. It is very common to observe such a review describing the grains structure, facies and other carbonate features. For further understanding of this geological perspective, there are some classic works approaching this matter: Folk (1959) (39), Folk (1962) (40), Dunham (1962) (34), Embry and Klovan (1971) (38), Ahr (2008) (2) as well as another thousands of other papers and articles addressing this subject. However, the focus is to understand how the changes in the carbonates framework can alter these rocks elastic attributes. Mainly, by talking about the changes related to diagenetic processes and petrophysical properties. For motivation purposes, Figure 2.1 shows the qualitative relation between fluids, petrophysical and rock properties with acoustic velocities in different scenarios.

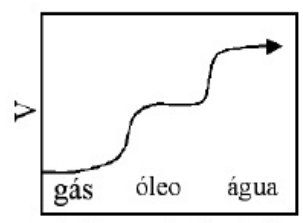

Módulo Bulk do Fluido
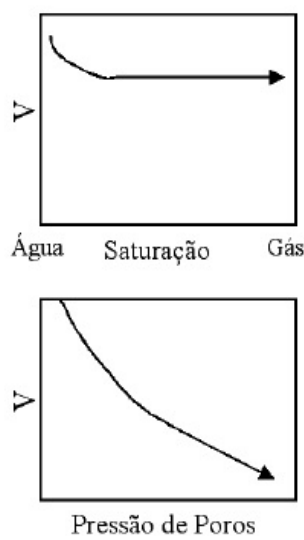
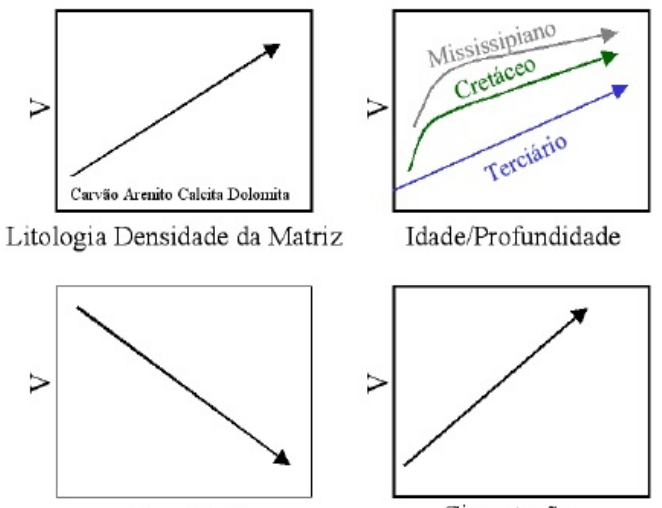

Porosidade

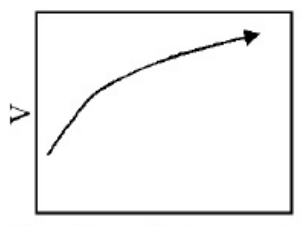

Pressão de Confinamento

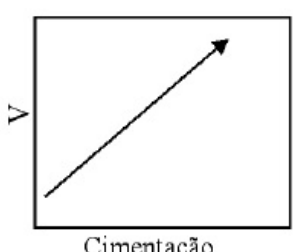

Cimentação

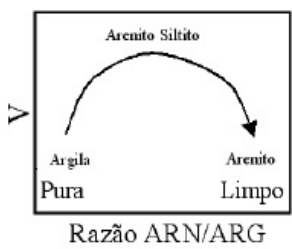

Figure 2.1: Qualitative relation between fluids, petrophysical and rock properties with acoustic velocities. Image from Mavko et al., (2009) (68).

In the same way that petrophysical properties affect the acoustic velocities, diagenetic processes also generate huge changes in the elastic responses. In the following bullets, some examples of Figure 2.1 are corroborated by some illustrations of how the rock elastic attributes can be affected in the matter of clay content, diagenetic processes and presence of different pore types in the rock framework. Besides that, it will be shown how rock physics models can be related to diagenetic changes for those examples, where siliclastic and 
carbonate rocks were described following - Avseth et al., (2005) (12) and Dvorkin et al., (2014) (30):

- Avseth et al., (2005) (12) present a good reason why this kind of subject - how the rock physics is related to the rock intrinsic properties - should gain special attention. In their book it is possible to see two examples of scattered $V_{p}-\phi$ crossplots even for siliciclastic and carbonates rocks. In order to explain these behaviors, Han (1986) (49) stated that the scattering problem of siliciclastic samples in $V_{p}-\phi$ crossplots is likely to be related to the clay content in the rock framework, whereas Anselmetti and Eberli (1997) (1) conducted a wide experiment with carbonate core samples and showed that the scattering in $V_{p}-\phi$ crossplots of carbonates was not related to clay content but to different pore shapes in the carbonate framework.

- In the same way, Dvorkin et al., (2014) (30), in an example in their book, state that the Raymer-Hunt-Gardner equation (Raymer et al., (1980) (75)) was a good fit for some siliciclastic rock samples whereas for other datasets the fit was not good, despite the fact that, both datasets had similar mineralogy and had similar amounts of clay, the velocities were very different. Ultimately, the hypothesis that one dataset was more affected by the diagenetic process of cementation than the other proved itself to be plausible.

Diagenetic processes really affect the rocks frameworks and by consequence change their elastic attributes. Quantifying and understanding those effects are highly important and great effort has been made since the early 80 's as can be seen with the modest and simple aforementioned examples. It is possible to state that rock physics have been used as an important tool to physically describe those effects and understand how they change the elastic attributes of the reservoirs for siliciclastic and even carbonate rocks. Currently, there are some new approaches, mainly regarding theoretical inclusion models, which represent the elastic responses of carbonate reservoirs quite well (Jakobsen (2003a,b) (54) (53) and Xu \& Payne (2009) (86)). In this way, in the next section, the main factors which alter the rock elastic attributes will be described taking into consideration the several aspects of: lithology, pore type, porosity, diagenetic processes and pressure. This chapter is limited to the carbonates point of view, and siliciclastic rocks will not be addressed with the same attention. 


\section{1}

\section{Main Factors which affect the Carbonate Rock Elastic Attributes}

The remarkable work of Anselmetti and Eberli (1993) () was one of the precursors to approach control factors of acoustic velocities in carbonate rocks with such a wide sampling - 210 core samples - from several places, taking into consideration lithologies which goes from unconsolidated carbonates mud until totally lithified limestones and making measurements under different confining and pore-fluid pressures. It was possible through this paper to relate acoustic velocities measurements to intrinsic features of carbonates, such as: mineralogy, porosity, pore types and density. Seven were the number of factors pointed out by the authors which affect the carbonate velocities and each one of those have different relevance and effects. Despite the fact that a wide number of core samples were used to conduct this study, it should be important to say that, at the first sight, the conclusions obtained might be related to the local carbonates used from the several places. However, more than 10 years later other articles still confirm almost all the same conclusions obtained by those authors at that time for different datasets (Eberli et al., (2003) and Kumar and Han (2005) $(37,59))$. One of the conclusions obtained from the aforementioned authors was that the changes in carbonate acoustic velocities are more directly related to the rock pore geometries and porosities. In fact, Figure 2.2 delivers a very good illustration of how the acoustic velocities in carbonate core samples vary taking into consideration the porosity, pore types and diagenetic processes.

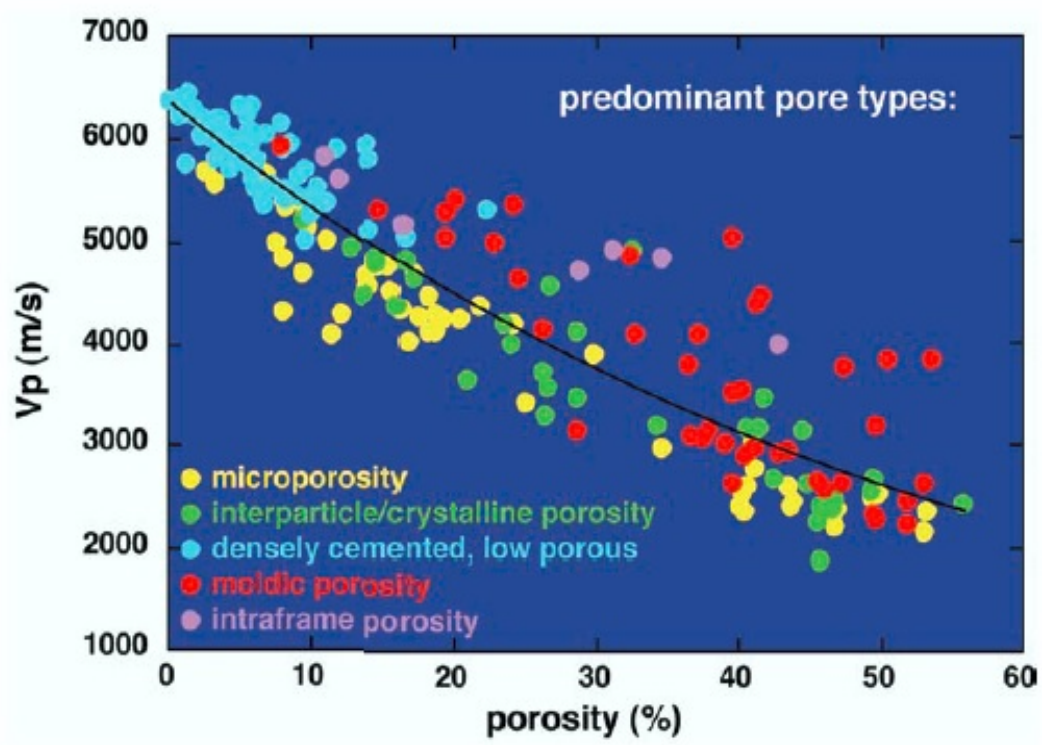

Figure 2.2: Crossplot $V_{p}-\phi$ for different carbonate core samples. Image from Eberli et al., (2003) (37). 
From Figure 2.2 some immediate conclusions can be quickly obtained:

- There is a high scattering in the crossplot and for one value of porosity the core samples have very different values of velocity;

- Microporosity and interparticle pores seem to reduce the carbonate rocks velocity whereas rounded pores or moldic increase the acoustic velocity;

- Samples highly cemented with low porosity also show high values of velocity. In fact this is very intuitive because low porosity and increase of cementation make the rock stiffer and consequently increase the acoustic velocities. Thus, the main point to notice in this bullet, is that the diagenetic processes also affect the carbonates elastic attributes.

Figure 2.2 brings meaningful insights about carbonates and their relations with $\mathrm{P}$ wave velocities and validates the conclusions of Anselmetti and Eberli (1993) () in a very didactic manner. In this way, the main conclusion, for now, is: pore types and diagenetic processes - in Figure 2.2 the cementation process is approached - change the carbonate elastic attributes. Therefore, in Figure 2.3, looking at some carbonate thin sections, it is possible to idealize those pore shapes as representations of ellipses with different formats.

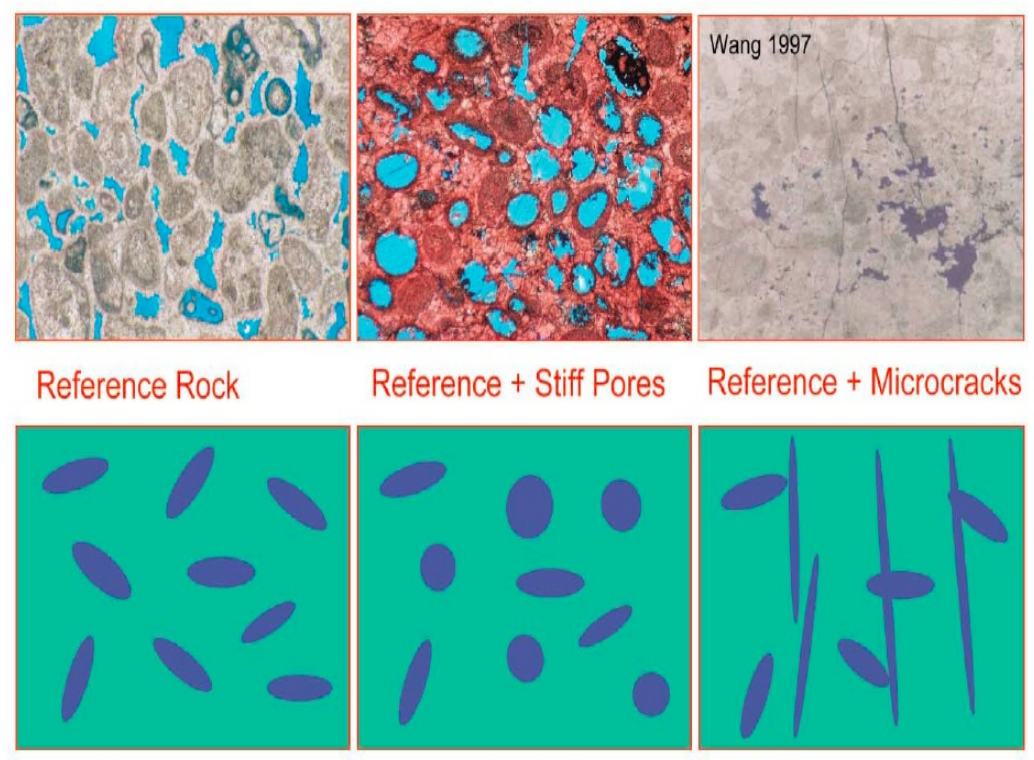

Figure 2.3: Carbonate rocks thin sections illustrating different pore types and their respective "ideal" representations. Image from Xu and Payne (2009) (86).

Figure 2.3 displays an important insight: carbonates pore types in different thin sections show geometric shapes similar to ellipses with different 
formats or different aspect-ratios $(\alpha)$. However, a bigger question should be asked: If Wang (1997) (90) already stated that the main pore types discovered in rocks are interparticle and intercrystalline, why rounded and crack pores are so wide spread in carbonate thin sections from all over the world? Figures 2.4 and 2.5, illustrate some rounded/moldic pores in carbonate thin sections regarding different sedimentological carbonate facies:
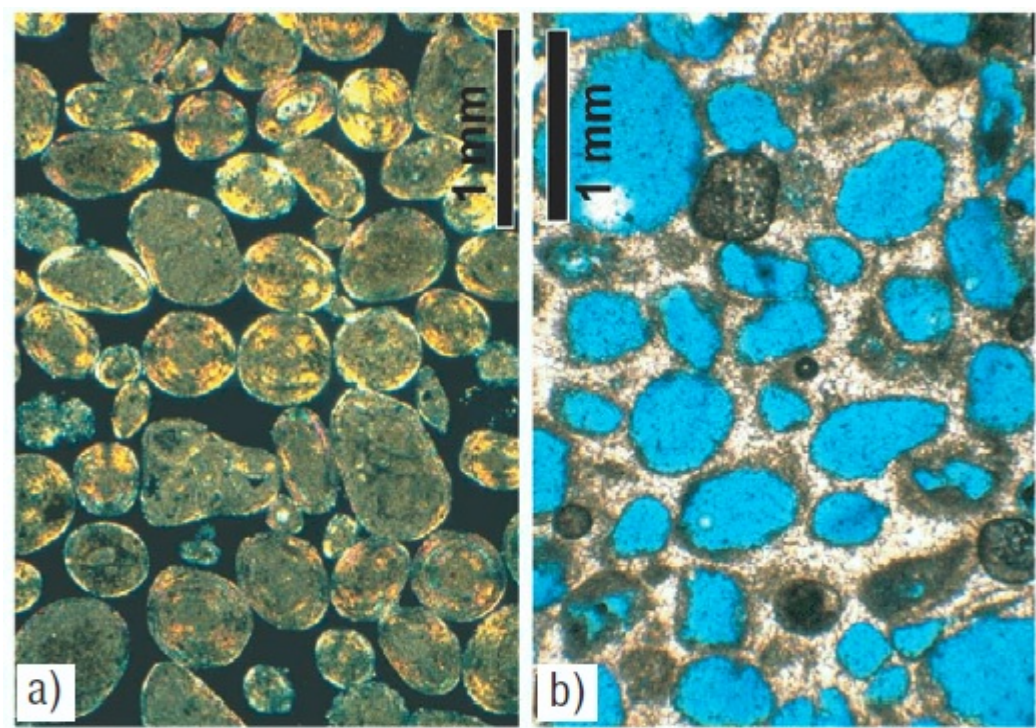

Figure 2.4: (a) oolithic carbonate sand with interparticle porosity and (b) diagenetic process of dissolution generating moldic porosity in the carbonate rock. Image from Eberli et al., (2003) (37).

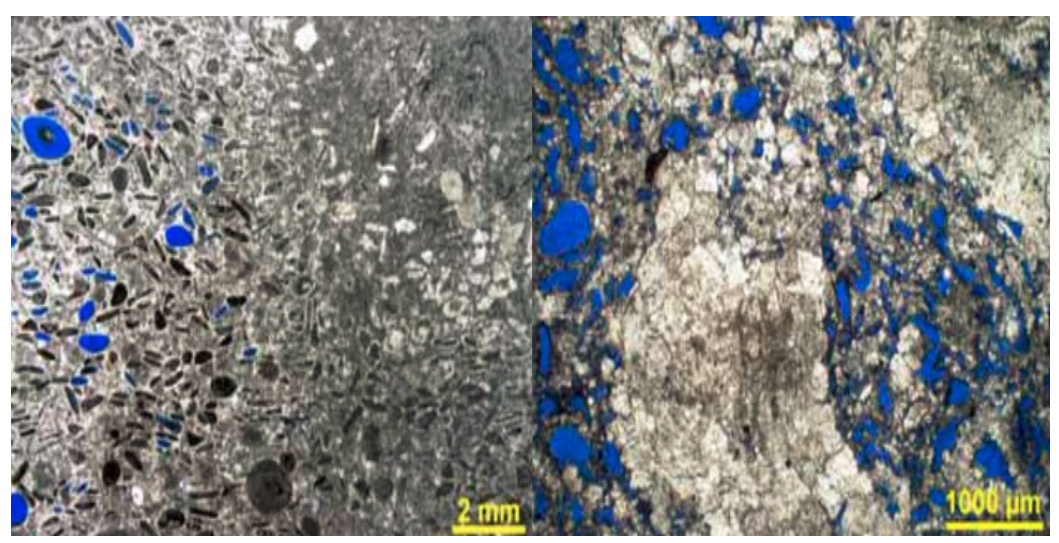

Figure 2.5: Moldic porosity generated through the diagenetic process of dissolution in both thin sections (mudstone and grainstone). Image from Bizotto (2014) (15).

Both figures illustrate the diagenetic process of dissolution in carbonate thin sections. Therefore, cementation as well as dissolution diagenetic processes 
(generation of moldic pores) highly affect the carbonate elastic attributes, as we can see in Figure 2.2, where the presence of rounded pore types are related to samples with high values of velocity. In fact, different from siliciclastic rocks, in which the pore space is mostly affected by mechanical compaction in shallow burial and quartz cementation for around 2 kilometers of depth (Avseth (2000) (11)), the carbonate rocks are highly affected by diagenetic processes, such as: dolomitization, cementation, dissolution, silicification and through some of those, an additional secondary porosity in the carbonates framework is generated.

It is beyond of this dissertation scope to approach and explain each one of those diagenetic processes. However, there is a wide range of literature addressing this subject, such as Bizotto (2014) (15), just to mention one of them. Taking into consideration that moldic porosity increases the carbonate acoustic velocities and this kind of porosity came from the changes of the primary porosity - interpaticle and intercrystalline -, how does the cracks porosity fits in this context? The genesis and comprehension of the crack pores in the carbonate rocks framework is as important as the understanding regarding the presence of moldic and interparticle pores because each one of them individually and they together have different roles in the changes of the carbonate elastic attributes as can be seen in Figure 2.6. This figure represents the template of the Xu and Payne (2009) (86) rock physics model which will be described in Chapter 3.

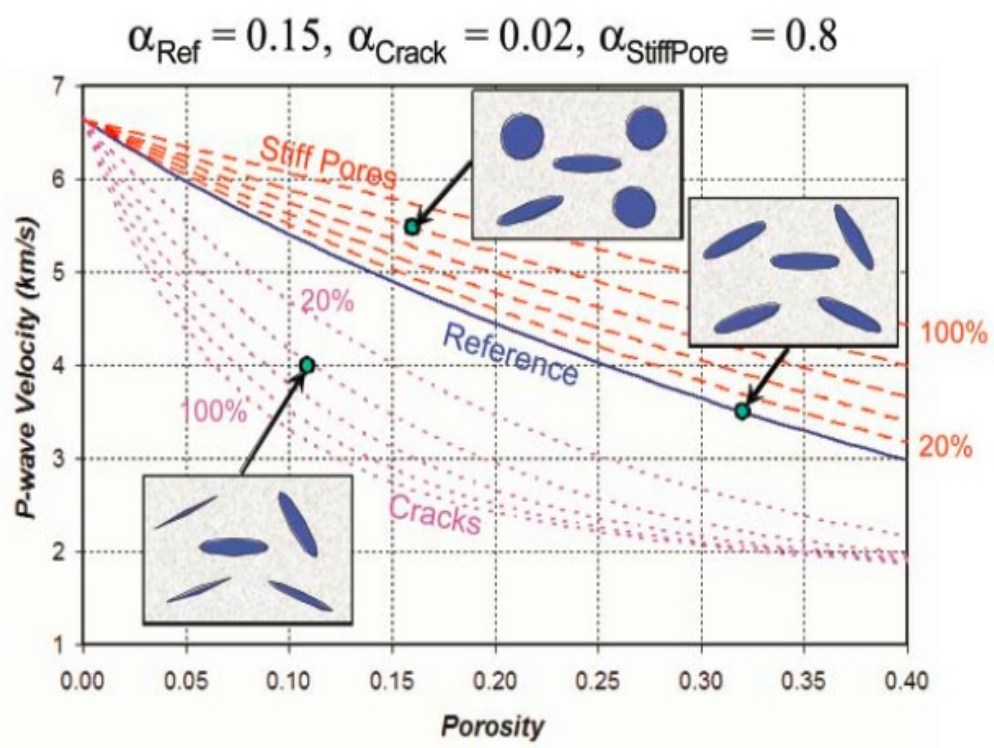

Figure 2.6: Distribution of pore types in crossplot $V_{p}-\phi$ in the rock physics template of Xu and Payne. Image from Xu and Payne (2009) (86). 
Regarding crack pores, in carbonate rocks, they can be represented by grain-to-grain contacts, microporosity (contacts between smaller particles) and microfractures. Moreover, it is important to mention that all these kind of crack porosities are responsible to reduce the carbonate stiffness (Baechle et al., (2008) (95)). Regarding crack pores representing microfractures, they are likely to be generated through fracturing processes and there are two main concerns to be considered related to effective pressure and pore pressure: (1) Generally, when the effective pressure increase with depth, the acoustic velocities also increase, closing the crack pores and making the rock stiffer and (2) on the other hand, where high anomalous pore-pressure values are found, the crack pores appear - this is likely to happen because some carbonates have a brittle behavior - and the acoustic velocities tend to decline because the presence of crack pores decreases the carbonate stiffness making the rock softer (Wang (1997) (90)). Microfractures are a special issue for carbonate rocks because some of those rocks have an intrinsic brittle nature and a fracture system can be very valuable for tight carbonates and even for tight sandstones in order to improve those reservoirs permeability (Xu and Payne (2009) (86)).

Nowadays, over-attention has been given to the pores aspect ratios in carbonate rocks, a plausible explanation for this is that porosity and pore-types are the main reasons which explain the expressive changes in the carbonate rocks velocities (Anselmetti and Eberli $(1997,1993,1999)(1$, , 9), Eberli et al., (2003) (37), Kumar and Han (2005) (59) and Kittridge (2015) (58)). Moreover, theoretical inclusion models take into consideration ellipsoidal inclusions with different aspect-ratios $(\alpha)$ (Xu and Payne (2009) and Jakobsen (2003a,b) $(86,54,53))$. In spite of that, some authors conducted studies about other possible geometric attributes in order to better describe the changes in the carbonates elastic attributes: Weger et al., (2004) and Baechle et al., (2004) $(88,16)$.

Weger et al., (2004) (88) address the importance of the following attributes combination: Roundness, PoA (Perimeter over Area) and DOMSize (Dominant Pore Size) which came from the digital image analysis. In this way, regarding their study, it was possible to conclude that the parameter PoA is the most important in the matter of affecting the velocities in carbonates for a specific porosity whereas DOMSize and roundness have less importance. On the other hand, some of the conclusions obtained by Baechle et al., (2004) (16) using DOMSize and P/Apor (Perimeter/Area Ratio) geometric factors for their dataset were: (1) samples with higher velocities and simple pore structure were defined by high values of DOMSize and low values of $\mathrm{P} / \mathrm{Apor}$ and (2) $\mathrm{P} /$ Apor has a direct relation with the tortuosity of the pore system, where 
higher values of $\mathrm{P} /$ Apor mean a higher tortuosity of the pore system.

This chapter is a brief review of rock physics of carbonates. The goal is to show how the carbonate rocks microstructure can affect their elastic attributes in an illustrative way. It was possible to notice that pore shapes and porosity are extremely important in the matter of velocity changes in carbonate rocks and diagenetic processes are also very relevant following the literature available. Besides that, those two features are somehow linked and related - e.g. moldic pores and diagenetic process of dissolution. Nevertheless, despite the fact that the pores aspect-ratios have shown a great value for several reasons, there are other geometric factors of carbonates that are also relevant which have been less addressed in a general manner. A much more extended and detailed explanation about all those aforementioned characteristics which affect the carbonate elastic attributes can be found in the classic chapter which Wang (1997) (90) wrote: Seismic Properties of Carbonates Rocks.

Ultimately, during this chapter, it was illustrated several carbonate sedimentary facies - oolithic grainstones, mudstones and others. However, during this dissertation, the only carbonate rock which will be addressed is the coquinas carbonate reservoir. In fact, in a few words, coquinas are carbonate rocks almost fully composed by shell debris which generally present a heterogeneous framework and are susceptible to diagenetic processes, such as: silicification, dolomitization and dissolution (Junior (2016) (43)). Regarding the Brazilian continental margin, these carbonate rocks represent important reservoirs because they are not only present in Campos Basin but also in Santos Basin in the Pre-Salt section together with other carbonate sedimentary facies (Bizotto (2014) (15)). 


\section{3 \\ Rock Physics Models Review}

This chapter is the cornerstone of the whole dissertation. The foundations of all rock physics models applied during this work will be addressed and described. However, it is important to make the following consideration: Despite the fact that one of the dissertation goals is to apply rock physics models in order to estimate the carbonates elastic attributes, it is relevant to mention that there are a lot of rock physics models that characterize different types of rocks: sandstones, shales and carbonates. It is beyond of this dissertation scope to describe and dive into each one of them, although a quick review with the due references is necessary.

The chapter two of Avseth et al., (2005) (12) explains in a very didactic manner the rock physics models for different kind of siliciclastic rocks. In fact, the most well-known rock physics models for clean sands are: The friable sand model, the contact sand model introduced by Dvorkin and Nur (1996) (31) and the constant-cement model - Avseth et al., (2000) (11) - which is a mathematical combination between the first two models previously mentioned (Avseth et al., (2005) (12)). In the same way that models for clean sands were already developed, there are rock physics models for shales, sandy shales, silty shales, shaly sands, and also for carbonates. As a matter of fact, rock physics models for source rocks have been highly developed - Guo et al., (2013) (44) even with the promise to reduce the uncertainty in AVO analysis (Avseth and Carcione (2015) (13)) and ultimately: theoretical inclusions models have been consistently used in rock physics models for carbonates (Xu and Payne (2009) $(86))$.

The basic premise of the rock physics models for clean sands is to take into consideration a regular pack of identical spheric grains which initially has a critical porosity that decreases due to the cementing or non-cementing particles or both processes (Dvorkin et al., (2014) (30)). This is especially important for siliciclastic rocks because under a shallow burial $(0-2 \mathrm{~km})$ the porosity loss in this kind of rock is mostly related to the mechanical compaction by the overburden layers above the reservoir rock, however, after $2 \mathrm{~km}$ of depth, the quartz cementation is likely to start and this diagenetic process really affects the elastic attributes of siliciclastic rocks (Avseth et al., (2000) (11)). On the 
other hand, rock physics models related to theoretical inclusions in a solid matrix - approaches mostly used to characterize carbonate rocks (Jakobsen $(2003 \mathrm{a}, \mathrm{b})$ and $\mathrm{Xu}$ and Payne $(2009)(54,53,86))$ - work in a different manner.

Inclusion models start with a theoretical elastic solid media with a zeroporosity endpoint and inclusions with different aspect ratios can be inserted in the solid matrix. This is a really different approach in comparison to the first one already mentioned for siliciclastics in the last paragraph, but it is a really good fit for carbonate rocks because pore shapes in carbonate rocks can be modeled by ellipses with different aspect ratios (Kumar and Han (2005), Abreu (2010), Allo et al., (2011) $(59,3,14))$, as described in Chapter 2. Despite of the comparison already made in the last paragraph and the specifics of each model for each kind of rock, Dvorkin et al., (2014) (30) warn that granular rock physics models can also be applied for carbonates pointing out a very good reason: sometimes granular structure is likely to appear in the carbonate rocks framework. This statement is partially true, in fact, it is really possible to apply those rock physics models for carbonates which have an apparent granular structure, however, it seems that granular rock physics models have not been used in this manner, such as:

- Allo et al., (2011) (14) applied the rock physics model of Xu and Payne (2009) (86) and the T-Matrix approach of Jakobsen $(54,53)$ for oolithic grainstones in an offshore carbonate field.

- Fournier and Codo (2016) (28) also addressed a non-specified rock physics model - built specifically for the Santos Basin - for spherulitic facies in the pre salt's carbonates.

In terms of classifying rock physics models, Avseth et al., (2005) (12) divided them into three general classes: Theoretical, Empirical and Heuristic. For a further and detailed understanding of those, the books Quantitative Seismic Interpretation - Applying Rock Physics Tools to Reduce Interpretation Risk written by the aforementioned authors as well as the classic The Rock Physics Handbook: Tools for Seismic Analysis of Porous Media of Mavko et al., (2009) (68) are very good choices. However, special attention will be given to the theoretical rock physics models and their following elastic models: Inclusion Models, Bounds and Transformations. In fact, theoretical models are also micromechanical models - Dvorkin et al., (2014) (30) - which try to represent the elastic, poroelastic or viscoelastic properties of a rock with reliability and confidence based on the rock microstructural information mineralogy, pore aspect ratios and fluids -, however, it is not easy to do such a task because three major pieces of information are needed to make accurate 
elastic attributes estimations of the rock through theoretical inclusion models (Mavko et al., (2009) (68)):

1. A quantitative estimation of the volume fraction of the constituents

2. The quantitative elastic moduli information of the phases inside the rock

3. The detailed geometric information of how the phases are settled in the rock framework relative to each other

When all the aforementioned information is available, it is very suggestive to use theoretical inclusion models. However, it is not always possible to obtain an accurate and reliable geometric arrangement of the rock phases as mentioned in the third item. Nevertheless, Avseth et al., (2005) (12) stated that there is an elegant way to face this kind of limitation using the "silent heroes" of rock physics models: The Elastic Bounds (Hashin-Shtrikman (1963) (51) and Voigt (1919) and Reuss (1929) bounds (83, 76)), which will be better explained in the next sections of this chapter along with the classic Gassmann's Relations (Gassman (1951) (42)); respectively, those models, match in the bounds and transformation elastic models.

At first glance, the rock physics model of $\mathrm{Xu}$ and Payne (2009) (86) and the T-Matrix approach of Jakobsen $(2003 \mathrm{a}, \mathrm{b})(54,53)$ seem to work in a similar manner because both of them are theoretical inclusion models and need to follow the three premises already aforementioned. However, they have some great differences between each other. In this way, trying to clarify the main differences between these rock physics models and pursuing a good way to explain how those approaches work, this chapter is divided into two main sections. The section 3.1 is related to the $\mathrm{Xu} \&$ Payne petroelastic model with its foundations and the section 3.2 addresses the T-Matrix rock physics model.

\section{1}

\section{Xu and Payne Rock Physics Model}

The rock physics model of Xu and Payne (2009) (86) for carbonate rocks is a continuity of the velocity-model of Xu and White (1995) (87) for clastic rocks. This approach is based on the creation of a theoretical elastic solid media with cavities/voids generated through inclusion models. Avseth et al., (2005) (12) stated that the inclusion models (e.g. the Kuster and Toksoz theory (1974a,b) (55) (56)) are responsible for inserting ellipsoidal or "penny-shaped" pore cavities in an elastic solid media which represents the mineralogical features of the rock. The new insight of the rock physics model of Xu and Payne is the multiple inclusions through the differential effective medium (DEM) 
model - Berryman (1992) (19) - together with the long-wavelength first-order theory of Kuster and Toksoz (1974a,b) (55) (56). Liu et al., (2009) (60) at that same time found out that to make accurate carbonates elastic attributes estimations through rock physics models, it is necessary a model capable to handle with different pore types. In this way, the total pore volume of the rock physics model of Xu and Payne can be divided into four: (1) clay-related pores, (2) interparticle pores, (3) microcracks, and (4) stiff pores, as follows:

$$
\phi_{T}=\phi_{\text {Clay }}+\phi_{\text {Crack }}+\phi_{\text {Interparticle }}+\phi_{\text {stiff }}
$$

- $\phi_{\text {Clay }}$ are the pores related to the possible presence of clay or silt in the carbonate rock taking into consideration the hypothesis of carbonaticclastic environment. Xu and White (1995) (87) had already stated that the proportion of $\phi_{\text {Clay }}$ is represented by the following relation:

$$
\phi_{\text {Clay }}=V_{s h} \phi_{T}
$$

where $V_{s h}$ is the shale volume and $\phi_{T}$ is the total porosity of the rock.

- $\phi_{\text {Crack }}$ can be related to fractures in the carbonate rocks and are highly sensitive to stress. The following equation expresses the relation between the crack porosity $\phi_{C r a c k}$ and the effective stress $\sigma_{e}$ :

$$
\phi_{\text {Crack }}=\phi_{\text {Init }} e^{-\beta \sigma_{e}}
$$

where $\beta$ is a constant related to the $\mathrm{P}$ and $\mathrm{S}$ wave velocities measured in the laboratory and $\phi_{\text {Init }}$ is the initial crack porosity in surface conditions ( $\sigma_{0}$ is equal to zero).

- $\phi_{\text {Interparticle }}$ are the most common pore types in sedimentary rocks. In carbonates rocks, they are generated in the early stages of diagenesis embedded in the primary porosity - Wang (1997) (90).

- $\phi_{\text {stiff }}$ are related to the moldic and vuggy pores present in the carbonate rock, most of the time those pores are generated through the diagenetic process of dissolution.

A special attention should be given for the $\phi_{\text {stiff }}$ and $\phi_{\text {Crack }}$ pore types. As a matter of fact, inclusion models are perturbed by cavities and when the 
volume of cavities increase, the overall rock elastic stiffness changes; then, the bulk and shear moduli also vary, altering the $\mathrm{P}$ and $\mathrm{S}$ wave velocities. This effect happens because the inclusions in the solid media are more compliant than the host elastic media itself (Avseth et al., (2005) (12)). Following Liu et al., (2009) (60), round pores in general, such as: vuggy and moldic pores $\left(\phi_{\text {stiff }}\right)$ tend to make the rock "faster", which means that this kind of pore increases the rock stiffness whereas flat pores with low aspect ratio - cracks $\left(\phi_{\text {Crack }}\right)$ - make the rock "weaker", which means that the rock stiffness decreases. In fact, that is the main reason why a scattered behavior is found in crossplots porosityvelocity in carbonates (Eberli et al., (2003) (37)). Moreover, in carbonate rocks, huge presence of rounded pores makes the $\mathrm{P}$ wave velocity increase whereas abundance of crack pores makes the $\mathrm{P}$ wave velocity decrease. Explaining in a didactic manner how the pore space of this rock physics model is composed is really important but for a further comprehension, the four main steps which compose the rock physics model of Xu and Payne (2009) (86) are described:

1. Generation of the effective solid elastic media through the bounds of Voigt (1910) (83) and Reuss (1929) (76) together with the mixing law of Hill (1952) (50) as known as Voigt-Reuss-Hill mean. This mean represents an average of the carbonate rock mineralogy which has been modeled (e.g., calcite, dolomite or even quartz).

2. The pore network of the elastic media is created through the DEM model (Berryman (1992) (19)) based on the Kuster and Toksoz (1974a,b) (55) (56) theory. It should be important to mention that it is possible to make different pore type inclusions and for special cases when the rock show high values of shale content, it is also possible to insert wet clay pores. Despite of that, the $\mathrm{Xu} \&$ Payne approach take into consideration dry inclusions and those voids are fulfilled with fluids later by the Gassmann's relations and inclusion based theory. The only exception is the clay related pores.

3. Apply a fluid mixing law to mix the possible mixture of fluids - brine, oil and/or gas - which are needed as inputs into the rock physics model, such as: The mixing fluid law of Wood (1955) (89). Initially, before applying the fluid mixture relation, the fluids properties are calculated by the well known Batzle and Wang (1992) (17) equations.

4. Ultimately, use the Gasmmann's relations for fluid substitution in the macropores of the elastic media already generated by the first two steps previously mentioned. Regarding the crack pores, the fluid saturation 
procedure happens through inclusion based-theory, assuming isolated micropores. In fact, $\mathrm{Xu} \&$ Payne when used the Gassmann's equations in order to saturate the crack pores, their rock physics model tended to underpredict $\mathrm{P}$ wave velocities. That is why the rock physics model of $\mathrm{Xu}$ \& Payne works with a mixed system of Gassmann-consistent macropores and non-Gassmann-consistent microcracks (Xu \& Payne (2009) (86)).

Before the explanation about the specifics of each elastic model inside the $\mathrm{Xu}$ and Payne rock physics model, in the next sections of this chapter; a brief comment will be delivered about the frequency issues inside this approach. While numbering and explaining the steps to run the $\mathrm{Xu}$ and Payne rock physics model, the application of Gassmann's fluid substitution was mentioned as a solution to transform a high frequency model because of the DEM approach to a low frequency model. Dvorkin et al., (2014) (30) explains that DEM modeling with fluid generates elastic attributes in a high frequency perspective - simulating ultrasonic laboratory measurements - because this theory states that there is no connection between the voids/inclusions. However, there is a simple workflow to run the $\mathrm{Xu}$ and Payne rock physics model in a low frequency perspective: Generate the dry effective moduli of the theoretical media and then make the Gassmann's fluid substitution to simulate low frequencies elastic responses. The Gassmann's relations are capable of doing such a task because this model strictly works for low frequencies taking into consideration that the wave induced pore pressures can be dissipated during a seismic period (Avseth et al., (12)). Nevertheless, Figure 3.1 describes in a very didactic way the four aforementioned steps of the $\mathrm{Xu} \&$ Payne model:

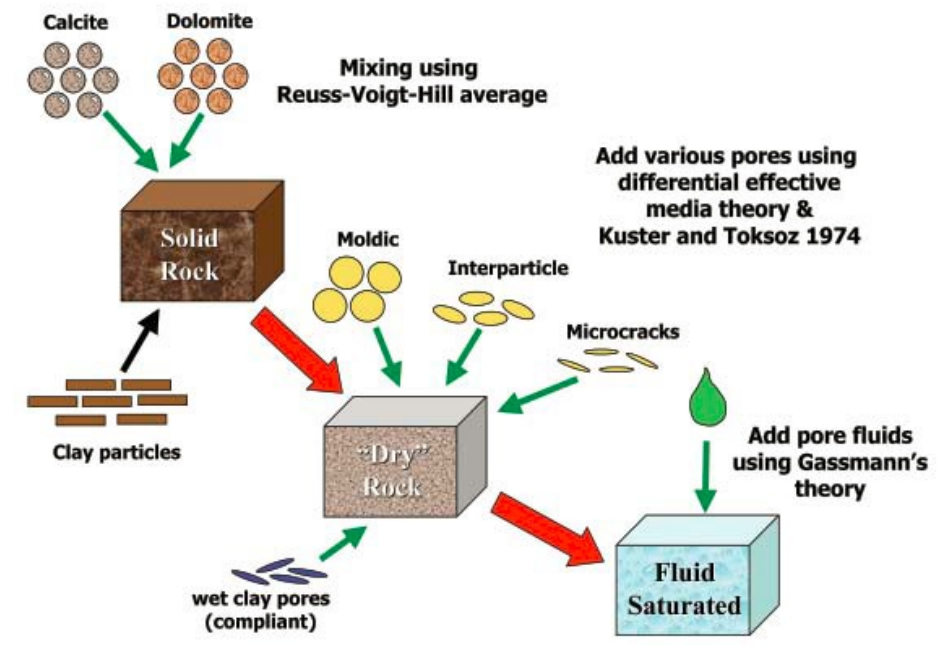

Figure 3.1: Diagram of the three steps of de Xu \& Payne rock physics model. Image from Xu and Payne (2009) (86). 


\subsection{1}

\section{Effective Solid Elastic Media}

\subsubsection{1}

\section{Voigt and Reuss Bounds}

Despite the fact that there is a consensus about the Hashin-Shtrikman bounds being the best choice to represent the elastic behavior of an isotropic linear elastic media (Avseth et al., (2005) (12) and Mavko et al., (2009) (68)), the workflow of $\mathrm{Xu}$ and Payne uses the Voigt-Reuss bounds as inputs for the mixing law of Hill. For given values of volume fractions of the mineral constituents and the respective elastic moduli of each one, The Voigt Upper Bound, $M_{V}$, of a mixture of $\mathrm{N}$ material phases, can be calculated as (Avseth et al., (2005) (12)):

$$
M_{V}=\sum_{i=1}^{N} f_{i} M_{i}
$$

where:

$f_{i}$ is the volume fraction of the $i$ th mineral constituent $M_{i}$ is the elastic modulus of the $i$ th mineral constituent

In a similar manner, for given values of volume fractions of the mineral constituents and the respective elastic moduli of each one, The Reuss Lower bound, $M_{R}$, of a mixture of $\mathrm{N}$ material phases, can be calculated as:

$$
\frac{1}{M_{R}}=\sum_{i=1}^{N} \frac{f_{i}}{M_{i}}
$$

$f_{i}$ is the volume fraction of the $i$ th mineral constituent $M_{i}$ is the elastic modulus of the $i$ th mineral constituent

The $M$ of Voigt and Reuss bounds can express any modulus: Bulk Modulus, Shear Modulus and others. However, it is more convenient - and 
makes more sense - to calculate the Voigt and Reuss averages of bulk and shear moduli: $M=K$ or $M=G$. In this way, through the bulk and shear moduli, obtain the others moduli through elasticity relations (Avseth et al., (2005) (12)).

\subsubsection{2}

\section{Voigt - Reuss - Hill Average}

In a few words, the Voigt-Reuss-Hill mean is the arithmetical mean of the Voigt-Reuss bounds, as follows (Hill (1952) (50)):

$$
M_{V R H}=\frac{M_{V}+M_{R}}{2}
$$

This computation is necessary to estimate the average of the minerals bulk and shear moduli which the rock is composed. This is a very useful information that can be used inside the rock physics model of $\mathrm{Xu}$ and Payne. Besides that, it is also possible to make a similar approach with the HashinShtrikman bounds as can be seen in Avseth et al., (2005) (12).

\subsection{2}

\section{Effective Moduli (Inclusion Methods)}

\subsubsection{1}

\section{Kuster and Toksoz Theory}

The approach of Kuster and Toksoz (1974a,b) (55) (56) is based on the long-wavelength first-order scattering theory. The authors goal with this article - which is divided into two parts: The first describes the theoretical formulations (Kuster and Toksoz (1974a) (55)) and the second makes the validation through experimental results (Kuster and Toksoz (1974b) (56)) was to obtain theoretical expressions to estimate the elastic attributes of a two phase-medium. The basic premises used by them in their models were two: (1) The wavelengths of the elastic waves which propagated through the two phase-medium were much higher than the size of a single inclusion and (2) the multiple scattering effects could be neglected. In this way, following Mavko et al., (2009) (68), it is possible to obtain the effective moduli $K_{K T}^{*}$ and $G_{K T}^{*}$ through the generalized expressions for different kind of pore shapes 
(Berryman (1992) (19)):

$$
\begin{gathered}
\left(K_{K T}^{*}-K_{m}\right) \frac{\left(K_{m}+\frac{4}{3} G_{m}\right)}{\left(K_{K T}^{*}+\frac{4}{3} G_{m}\right)}=\sum_{i=1}^{N} x_{i}\left(K_{i}-K_{m}\right) P^{m i} \\
\left(G_{K T}^{*}-G_{m}\right) \frac{\left(G_{m}+\zeta_{m}\right)}{\left(G_{K T}^{*}+\zeta_{m}\right)}=\sum_{i=1}^{N} x_{i}\left(G_{i}-G_{m}\right) Q^{m i}
\end{gathered}
$$

where:

$$
\zeta=\frac{G_{m}}{6} \frac{\left(9 K_{m}+8 G_{m}\right)}{\left(K_{m}+2 G_{m}\right)}
$$

$K_{m}$ and $G_{m}$ are, respectively, the bulk and shear moduli of the matrix which compose the elastic solid media. The coefficients $P^{m i}$ and $Q^{m i}$ are related to the inclusions material $i$ inside of the framework $m$ (geometrical factors). There are also standards coefficients $\mathrm{P}$ and $\mathrm{Q}$ for some specific shapes - aspect ratios - as it can be seen in Berryman (1980) (18) and Mavko et al., (2009) (68). However, the geometrical factors P and Q for arbitrary aspect ratios can be obtained through the following equations:

$$
P=\frac{1}{3} T_{i i j j}
$$

$$
Q=\frac{1}{5}\left(T_{i j i j}-\frac{1}{3} T_{i i j j}\right)
$$


where:

$$
T_{i i j j}=\frac{3 F_{1}}{F_{2}}
$$

$$
T_{i j i j}-\frac{1}{3} T_{i i j j}=\frac{2}{F_{3}}+\frac{1}{F_{4}}+\frac{F_{4} F_{5}+F_{6} F_{7}-F_{8} F_{9}}{F_{2} F_{4}}
$$

Therefore:

$$
\begin{gathered}
F_{1}=1+A\left[\frac{3}{2}(f+\theta)-R\left(\frac{3}{2} f+\frac{5}{2} \theta-\frac{4}{3}\right)\right] \\
F_{2}=1+A\left[1+\frac{3}{2}(f+\theta)-\frac{1}{2} R(3 f+5 \theta)\right]+B(3-4 R)+\frac{1}{2} A(A+3 B)(3-4 R)\left[f+\theta-R\left(f-\theta+2 \theta^{2}\right)\right] \\
F_{3}=1+A\left[1-\left(f+\frac{3}{2} \theta\right)+R(f+\theta)\right] \\
F_{4}=1+\frac{1}{4} A[f+3 \theta-R(f-\theta)] \\
F_{5}=A\left[-f+R\left(f+\theta-\frac{4}{3}\right)\right]+B \theta(3-4 R) \\
F_{6}=1+A[1+f-R(f+\theta)]+B(1-\theta)(3-4 R)
\end{gathered}
$$




$$
\begin{gathered}
F_{7}=1+\frac{1}{4} A[3 f+9 \theta-R(3 f+5 \theta)]+B \theta(3-4 R) \\
F_{8}=A\left[1-2 R+\frac{1}{2} f(R-1)+\frac{1}{2} \theta(5 R-3)\right]+B(1-\theta)(3-4 R) \\
F_{9}=A[(R-1) f-R \theta]+B \theta(3-4 R)
\end{gathered}
$$

Thus, we can obtain the parameters A, B and R as follows:

$$
\begin{gathered}
A=\frac{G_{i}}{G_{m}}-1 \\
B=\frac{1}{3}\left(\frac{K_{i}}{K_{m}}-\frac{G_{i}}{G_{m}}\right) \\
R=\frac{\left(1-2 \nu_{m}\right)}{2\left(1-\nu_{m}\right)}=\frac{G_{m}}{K_{m}+\frac{4}{3} G_{m}}
\end{gathered}
$$

Finally, the $\theta$ and $f$ functions can be obtained for prolate $(\alpha<1)$ and/or oblate spheroids $(\alpha>1)$, respectively:

$$
\theta=\left\{\begin{array}{l}
\frac{\alpha}{\left(\alpha^{2}-1\right)^{\frac{3}{2}}}\left[\alpha\left(\alpha^{2}-1\right)^{\frac{1}{2}}-\cosh ^{-1}(\alpha)\right] \\
\frac{\alpha}{\left(1-\alpha^{2}\right)^{\frac{3}{2}}}\left[\cos ^{-1}(\alpha)-\alpha\left(1-\alpha^{2}\right)^{\frac{1}{2}}\right]
\end{array}\right.
$$

with: 


$$
f=\frac{\alpha^{2}}{1-\alpha^{2}}(3 \theta-2)
$$

During this section it was demonstrated how to calculate the geometrical factors $\mathrm{P}$ and $\mathrm{Q}$ for arbitrary aspect ratios. However, in the $\mathrm{Xu}$ and Payne approach, for clean carbonate rocks, three different dry pore types with their volume proportions are considered to generate the porous media in the rock physics modeling: $\alpha_{\text {stiff }}, \alpha_{\text {reference }}, \alpha_{\text {cracks }}$ or $\alpha_{S}, \alpha_{R}, \alpha_{C}$ and their respective volume fractions $V_{S}, V_{R}, V_{C}$. In this way, it is necessary to follow the Keys and $\mathrm{Xu}$ (2002) (85) recipe to calculate the resultant geometrical factors $\mathrm{P}$ and $\mathrm{Q}$ when more than one aspect ratio is considered. Therefore, after obtaining the constants $F_{1}-F_{9}$ for each pore type $\alpha_{S}, \alpha_{R}, \alpha_{C}$, it is possible to compute the aspect ratio functions $T_{i i j j}\left(\alpha_{l}\right)$ and $F\left(\alpha_{l}\right)$, as follows:

$$
\begin{gathered}
T_{i i j j}\left(\alpha_{l}\right)=T_{i i j j}=\frac{3 F_{1}}{F_{2}} \\
F\left(\alpha_{l}\right)=T_{i j i j}-\frac{1}{3} T_{i i j j}=\frac{2}{F_{3}}+\frac{1}{F_{4}}+\frac{F_{4} F_{5}+F_{6} F_{7}-F_{8} F_{9}}{F_{2} F_{4}}
\end{gathered}
$$

Where:

$$
\alpha_{l}=\left[\begin{array}{c}
\alpha_{S} \\
\alpha_{R} \\
\alpha_{C}
\end{array}\right] V_{l}=\left[\begin{array}{c}
V_{S} \\
V_{R} \\
V_{C}
\end{array}\right]
$$

Thus, it is possible to calculate the resultant geometric factors $\mathrm{P}$ and $\mathrm{Q}$ related to $\alpha_{S}, \alpha_{R}, \alpha_{C}$ and $V_{S}, V_{R}, V_{C}$, as follows (Keys and $\mathrm{Xu}$ (2002) (85)): 


$$
\begin{gathered}
P=\frac{1}{3} \sum_{l=S, R, C} V_{l} T_{i i j j}\left(\alpha_{l}\right) \\
Q=\frac{1}{5} \sum_{l=S, R, C} V_{l} F\left(\alpha_{l}\right)
\end{gathered}
$$

In the next section, it will be possible to understand how those resultant geometrical factors are inputted in the DEM's analytical solutions.

\subsubsection{2}

\section{Differential Effective Medium Theory (DEM)}

The differential effective medium theory (DEM) is embedded in the socalled: Self-consistent approximations of effective moduli. Berryman (1980) (18) presented an important paper approaching this matter and also proposed - at that time - a new self-consistent method to calculate elastic attributes of theoretical elastic media with ellipsoidal inclusions based on the elastic-wave scattering theory. Some of the conclusions obtained from this article were that the self-consistent model - for all ellipsoidal shapes - proposed was capable of satisfying the elastic bounds, such as: Hashin - Shtrikman. Furthermore, it was also proved that this approach was superior in comparison with other self-consistent models.

The DEM theory works starting from the endpoint where there is only the rock matrix without any kind of porosity, and this matrix - $K$ and $G-$ is computed by some mixture law, such as: Hill's Average. It means that the initial condition for $\phi=0$ is known: The bulk and shear moduli of the matrix (phase 1) are the mineral bulk and shear moduli already given by the average of Hill. However, the main point is to calculate the bulk and shear moduli $K^{*}$ and $G^{*}$ - with the desired additional inclusions (phase 2) in the theoretical matrix, that can be fulfilled or dry. In this way, it is possible to obtain the effective bulk and shear moduli $-K^{*}$ and $G^{*}-$, respectively, through the following ordinary differential equations (ODEs) system (Berryman (1992) (19)): 


$$
\begin{aligned}
& (1-y) \frac{d K^{*}(y)}{d y}=\left(K_{2}-K^{*}(y)\right) P^{* 2} \\
& (1-y) \frac{d G^{*}(y)}{d y}=\left(G_{2}-G^{*}(y)\right) Q^{* 2}
\end{aligned}
$$

Thus, the initial conditions are $K^{*}(0)=K_{1}$ and $G^{*}(0)=G_{1}$, taking into consideration that $K_{1}$ and $G_{1}$ are the bulk and shear moduli of the rock matrix (phase 1) whereas $K_{2}$ and $G_{2}$ are the bulk and shear moduli of the inclusions (phase 2). $P^{* 2}$ and $Q^{* 2}$ are the geometrical factors of the inclusions (phase 2). $y$ is the concentration of the inclusions (phase 2) and for fluid inclusions or voids: $y=\phi$, as follows:

$$
\begin{aligned}
& (1-\phi) \frac{d K^{*}(\phi)}{d \phi}=\left(K_{2}-K^{*}(\phi)\right) P^{* 2} \\
& (1-\phi) \frac{d G^{*}(\phi)}{d \phi}=\left(G_{2}-G^{*}(\phi)\right) Q^{* 2}
\end{aligned}
$$

Therefore, the following analytical solutions of the ordinary differential equations (ODEs) for dry inclusions are obtained when $y=\phi$ following Keys and $\mathrm{Xu}$ (2002) (85):

$$
\begin{aligned}
& K(\phi)=K_{0}(1-\phi)^{P} \\
& G(\phi)=G_{0}(1-\phi)^{Q}
\end{aligned}
$$

In this way, $K_{0}$ and $G_{0}$ are the bulk and shear moduli of the rock matrix and the terms $P$ and $Q$ are the resultant geometrical factors (explained in the 
previous section - 3.1.2.1) for the ODEs and their analytical solutions. The geometric factors of the inclusions can be obtained through standard equations for specific pore types (Mavko et al., (2009) (68)) or can be calculated through the Kuster and Toksoz $(1974 a, b)(55,56)$ relations for any pore type with any values of aspect ratio $(\alpha)$ as can be seen in section 3.1.2.1.

\subsection{3}

\section{Fluids}

\subsubsection{1}

\section{Wood's Mixing Law}

Before starting the explanation of how important the mixing law of Wood (1955) (89) is in the approach of $\mathrm{Xu}$ and Payne (2009) (86), it is necessary to point out that the Batzle and Wang (1992) (17) relations also play an important role in the matter of fluids calculations in other perspective. In fact, those relations were validated with a good match regarding laboratory measurements (Vasquez and Dillon, (1993) (45)). Therefore, through the equations of Batzle and Wang, accurate values of the elastic moduli of fluids: brine, oil and gas, can be obtained regarding the variations of pressure, temperature, salinity among others. In spite of this, when the fluids in situ information is available, such as: Bulk modulus $\left(K_{f l}\right)$ and density $\left(\rho_{f l}\right)$ and it is necessary to make a fluid mixture, it is very suggestive to use the Wood's Mixing Law to calculate the $\mathrm{P}$ wave acoustic velocity of the mixture $\left(V_{f l}\right)$, as follows:

$$
V_{f l}=\sqrt{\frac{K_{f l}}{\rho_{f l}}}
$$

Therefore, this equation is valid when the wavelength is much higher than the heterogeneities present in the rock itself. Thus, the bulk modulus can be calculated through the Reuss (1929) (76) bound/average whereas the density can be calculated with the Voigt (1910) (83) bound/average:

$$
\frac{1}{K_{f l}}=\sum_{i=1}^{N} \frac{f_{i}}{K_{i}}
$$




$$
\rho_{f l}=\sum_{i=1}^{N} f_{i} \rho_{i}
$$

Where $f_{i}$ is the fraction of each kind of fluid and $K_{i}$ and $\rho_{i}$ are the respective bulk modulus and density of each fluids phase.

\subsubsection{2}

\section{Gassmann's Relations}

The Gassmann's relations (Gassmann (1951) (42)) have a simple but noble and important goal: How the elastic moduli of a dry or saturated rock changes if the fluids are added or changed/replaced in the rock framework. This is the fluid substitution problem that can be expressed by the following equation:

$$
\frac{K_{s a t}}{K_{m}-K_{s a t}}=\frac{K_{d r y}}{K_{m}-K_{d r y}}+\frac{K_{f l}}{\phi\left(K_{m}-K_{f l}\right)}
$$

And after some manipulations (Mavko et al., (2009) (68)):

$$
K_{s a t}=K_{d r y}+\frac{\left(1-\frac{K_{d r y}}{K_{s a t}}\right)^{2}}{\frac{\phi}{K_{f l}}+\frac{1-\phi}{K_{m}}-\frac{K_{d r y}}{K_{m}^{2}}}
$$

Where:

$$
G_{\text {sat }}=G_{d r y}
$$




$$
\rho_{\text {sat }}=(1-\phi) * \rho_{m}+\phi * \rho_{f l}
$$

Where $K_{\text {sat }}$ is the effective bulk modulus of the rock with the saturating fluid, $K_{d r y}$ is the effective bulk modulus of the dry rock, $K_{f l}$ is the effective bulk modulus of the saturating fluid (pore fluid), $K_{m}$ is the effective bulk modulus of the matrix or mineral which the rock is composed of, $G_{d r y}$ is the effective shear modulus of the dry rock, $G_{s a t}$ is the effective shear modulus of the saturated rock, $\phi$ is the porosity of the rock, $\rho_{\text {sat }}$ is the density of the saturated rock, $\rho_{m}$ is the matrix density and $\rho_{f l}$ is the pore fluid density. For a further understanding about the Gassmann's relations, it is worthy to say that Avseth et al., (2005) (12) make a detailed and didactic fluid substitution analysis regarding this rock physics model addressing its pitfalls, cautions and limitations.

It is important to remind that the Gasmmann's relations for fluid substitution are applied in the macropores of the elastic media in the $\mathrm{Xu}$ \& Payne workflow. Regarding the crack pores, the fluid saturation procedure happens through inclusion based-theory, assuming isolated micropores. In fact, Xu \& Payne when used the Gassmann's equations in order to saturate the crack pores, their rock physics model tended to underpredict $\mathrm{P}$ wave velocities. That is why the rock physics model of $\mathrm{Xu} \&$ Payne works with a mixed system of Gassmann-consistent macropores and non-Gassmann-consistent microcracks (Xu \& Payne (2009) (86)).

\section{2}

\section{T-Matrix Rock Physics Model}

The T-Matrix approach of Jakobsen et al., (2003a,b) $(54,53)$ is based on physically transparent integral equation methods (Green's Function Techniques) which have a strong and heavy mathematical foundation behind the scenes that enables to work with the effects of fluid flow between cavities when calculating the elastic media attributes. Moreover, it is also possible to make inclusions of minerals or solid constituents and connected or disconnected cavities with different shapes, orientations and spacial distributions. This viscoelastic effective medium theory in comparison with other approaches, has a special advantage: the T-Matrix approach delivers a frequency-dependent inclusion model that takes into consideration all frequencies - infrasonic, sonic and ultrasonic -, in an attempt to cover all the flaws related to frequency 
of others rock physics models. On the other hand, the Kuster and Toksoz (1974a) (55) approach works as a high-frequency theoretical inclusion model that takes into consideration isolated pores as well as the DEM theory of Berryman (1992) (19), whereas the rock physics model of Xu and Payne works for low-frequencies due to the presence of Gassmann's relations inside of the model workflow. Nevertheless, during this chapter, it was possible to notice that there is a variety of rock physics models and plenty of differences can be found among each one of them. In this way, one of the dissertation goals is to evaluate the elastic attributes estimations of the rock physics models of $\mathrm{Xu}$ and Payne and T-Matrix. Therefore, between these two rock physics models, the main differences and similarities are described, as follows:

- Both of them can model the porous media of the initial composite through ellipsoidal inclusions of aspect ratio $(\alpha)$

- In terms of frequency, naturally, the rock physics model of Xu and Payne would be a high frequency rock physics model because the inclusions are made through the DEM theory grounded in the long-wavelength firstorder approach of Kuster and Toksoz. However, due to the Gassmann's fluid substitution, a low-frequency model prevails. On the other hand, the rock physics model of Jakobsen et al., (2003a,b) $(54,53)$ is not based on the Gassmann's relations and takes into consideration all the frequencies needed (infrasonic, sonic and ultrasonic). In fact, that is one of the most prominent advantages of the T-Matrix model over the $\mathrm{Xu} \&$ Payne model taking into consideration the relevance of elastic attributes changes regarding the frequency in the quantitative seismic interpretation scope.

- For both rock physics models, inclusions with different pore shapes can be made. However, through the T-Matrix rock physics model, it is possible to build the pore network of the elastic solid media with connected and/or disconnected pores. In this way, it is possible to simulate a family of pores as connected and another family of pores as disconnected, in the same pore network, inside of the theoretical elastic media. Therefore, when all the pores in the T-Matrix approach are connected, it is possible to simulate a low frequency model where the wave induced pore pressures can be dissipated during the seismic period whereas when all the pores are disconnected a high frequency model is simulated. On the other hand, in the $\mathrm{Xu}$ and Payne approach, it was 
proven when the Gassmann's relations were applied in crack pores, the wave induced pore pressures could not equilibrate in a seismic period. Thus, this issue led a violation one of the Gassmann's assumptions. In this way, the rock physics model of $\mathrm{Xu}$ and Payne takes into consideration macropores as perfectly connected and microcracks as disconnected.

- The T-Matrix approach only uses inclusion based theory in order to saturate the dry elastic media. On the other hand, the $\mathrm{Xu}$ \& Payne rock physics model uses the Gassmann's relations in order to saturate macropores and inclusion based theory to saturate crack pores, as it was possible to see in the section 3.1 .

In the thesis of Agesborg (2007) (6) the T-matrix approach of Jakobsen et al., (2003a,b) $(54,53)$ was applied specifically for carbonates rocks in different manners. Four articles came up from the thesis and special attention should be given to the following: Agesborg et al., (2008) and Agesborg et al., (2009) $(7,5)$. In both articles, dual porosity systems to investigate velocities behaviors in carbonate rocks were addressed. Despite the fact that it is likely to assume that the dry and saturated shear moduli are equal for a given rock since the fluids have no shear modulus, Agesborg et al., (2008) (7) discovered that shear strengthening may occur in carbonate rocks due to the squirt flow effects generated by wave-induced fluid flow events. This conclusion corroborates analyses previously made by other authors who state that shear weakening or strengthening can happen in carbonate rocks. Nevertheless, Agesborg et al., (2009) (5) used the T-matrix approach to investigate the fluids effects in velocities of carbonate rocks and then, a comparison with the Gassmann's relations was made showing that the deviations varied from close to zero until five percent. Finally, after this brief description and introduction, part of the mathematical formalism of the frequency-dependent inclusion model of Jakobsen et al., (2003a,b) (53) will be presented. In this way, during this chapter, only the most important equations will be addressed in order to understand how this rock physics model works in a general manner and for further details, it is advisable to study the articles which are the foundations of this section.

\subsection{1}

\section{T-Matrix Mathematical Formulations}

The T-Matrix approach of Jakobsen et al., $(2003 \mathrm{a}, \mathrm{b})(54,53)$ is a frequency-dependent theoretical inclusion model which takes into consideration a homogeneous elastic solid media embedded of inclusions. When the 
inclusions have the same shapes and orientation they are divided in families labeled $r$. In this way, following the mathematical arrangement of Agesborg et al., (2005) (4), the stiffness tensor $C^{*}$ of the elastic solid media can be described in the following manner:

$$
C^{*}=C^{(0)}+C^{(1)}:\left(I_{4}+C_{1}^{-1}: C_{2}\right)^{-1}
$$

where:

$$
C_{1}=\sum_{r}^{N} v^{(r)} t^{(r)}
$$

$$
C_{2}=\sum_{r=1}^{N} \sum_{s=1}^{N} v^{(r)} t^{(r)} G_{d}^{(r s)} t^{(s)} v^{(s)}
$$

Therefore, $C^{(0)}$ is the fourth-rank stiffness tensor of the homogeneous elastic media, $I_{4}$ is the identity for fourth-rank tensors, $v^{(r)}$ in the concentration of inclusions of a family $r, t^{(r)}$ is the t-matrix of the inclusions of the family $r, G_{d}^{(r s)}$ is obtained through the strain Green's function over a characteristic ellipsoid. Nevertheless, the T-Matrix formulation for an isolated inclusion of a family/type $r$, dependent on a frequency $w$, can be written as:

$$
t^{(r)}(\omega)=\left(C^{(r)}(\omega)-C^{(0)}\right):\left[I_{4}-G^{(r)}:\left(C^{(r)}(\omega)-C^{(0)}\right)\right]^{-1}
$$

Where the $G^{(r)}$ is a fourth-rank tensor which depends on $C^{(0)}$ and the geometric details - shape/orientation/aspect ratio - of the family of inclusions $r$.

If: 


$$
\begin{gathered}
C^{(r)}=w=0 \\
t^{(r)}=t_{d}^{(r)}
\end{gathered}
$$

Thus, it is possible to conclude that a dry inclusion has been modeled in the elastic media and the t-matrix of a dry inclusion is obtained $\left(t_{d}^{(r)}\right)$. On the other hand, the t-matrix formulation for connected inclusions in the theoretical elastic solid media can be calculated as:

$$
t^{(r)}=t_{d}^{(r)}+\frac{\Theta Z^{(r)}+i \omega \tau \kappa_{f} X^{(r)}}{1+i \omega \gamma^{(r)} \tau}
$$

Thus:

$$
\begin{gathered}
S^{(0)}=\left(C^{(0)}\right)^{-1} \\
X^{(r)}=t_{d}^{(r)}: S^{(0)}:\left(I_{2} \otimes I_{2}\right): S^{(0)}: t_{d}^{(r)} \\
\Theta=\kappa_{f}\left(\left(1-\kappa_{f} S_{u u v v}^{(0)}\right)\left(\sum_{n=1}^{N_{c}} \frac{v^{(r)} t_{d}^{(r)}}{1+i \omega \gamma^{(r)} \tau}\right)+\epsilon_{d}^{(r)}: S^{(0)}:\left(\sum_{n=1}^{N_{c}} \frac{v^{(r)\left(K_{d}^{(r)}\right) u u v v} t_{d}^{(r)}}{1+i \omega \gamma^{(r)} \tau}\right)-\frac{i k_{u} k_{v} \Gamma_{u v} \kappa_{f}}{\eta_{f} \omega}\right)^{-1}:\left(\sum_{n=1}^{N_{c}} \frac{v^{(n)} t_{d}^{(n)}}{1+i \omega \gamma^{(n)} \tau}\right)
\end{gathered}
$$


And:

$$
\begin{gathered}
\gamma^{(r)}=1+\kappa_{f}\left(K_{d}^{(r)}-S^{(0)}\right)_{u u v v} \\
K_{d}^{(r)}=\left(I_{4}-G^{(r)}: C^{(0)}\right)^{-1}: S^{(0)}
\end{gathered}
$$

Where $\kappa_{f}$ and $\eta_{f}$ are the fluid bulk modulus and viscosity, respectively, $I_{2}$ is a second rank tensor, $k_{u}$ and $k_{v}$ are the components of the wave number vector, $\Gamma_{u v}$ is the rock permeability tensor and $\tau$ is the relaxation time constant. Following Jakobsen et al., (2003a,b) (53), the relaxation time constant $(\tau)$ is empirically calculated and depends on several properties, such as: pore shapes, quantity of cracks, fluid information and matrix mineralogy. Agesborg (2007) (6) makes a brief but good and didactic review about the relaxation constants in the literature approaching the specifics of each one and when each equation should be used depending on the information available. 


\section{4}

\section{Bayesian Inference Theory}

The stochastic or probabilistic approach through the Bayesian Inference has been a trend in many fields of research (Vrugt (2016) (84)). Therefore, regarding geophysical applications, in the earlier 2000's, Loures (2001) (21) already applied this approach for reservoir characterization purposes linking well log and seismic data to rock physics models (Loures and Moraes (2002) (20)). Nevertheless, in the recent years, this subject has not been stressed enough and the Bayesian framework has been posed in many ways trying to better approach different problems regarding not only the application in rock physics models and seismic reservoir characterization workflows (Grana (2016) (66) and Figueiredo et al., (2017) (65)) but also in other fields, such as: Hydrology, Engineering and others (Scharnagl et al., (2011), Scharnagl et al., (2011) and Vrugt (2016) (23, 22, 84)). As a matter of fact, Vrugt et al., (2008a, 2009a) (35, 33) developed a MATLAB toolbox that is based on the Markov Chain Monte Carlo (MCMC) simulation and the DiffeRential Evolution Adaptive Metropolis (DREAM) algorithm which has been used in several research fields and provides means to solve posterior sampling problems through the Bayesian inference using MCMC simulations.

The possibility of posing the rock physics models described in Chapter 3 in a Bayesian framework is paramount in order to obtain a better evaluation of those models inputs and outputs which are not represented anymore by deterministic values, but by probability density functions (PDFs). During this work it will be possible to see that both rock physics models require a set of input parameters which are very uncertain. Likewise, the outputs tend to be also uncertain. In this way, using different inputs inside of rock physics models, similar outputs can be obtained. Therefore, to address and better understand this non-uniqueness problem, in the evaluation of both rock physics models, the uncertainties related to the measurements needed as inputs as well as the geological uncertainties due to indirect measurements will be settled in a Bayesian framework. Thus, it will be possible to achieve a more reliable evaluation of both rock physics models regarding elastic attributes estimations of the coquinas reservoir. 
This chapter goal is not to make a detailed and complete review about Bayesian Inference, Monte Carlo Simulation and Markov Chains. In fact, there is extensive literature approaching all those subjects and it would be too exhaustive to describe the foundations of each one during this chapter (e.g. Vrugt (2016) (84) and Brooks (1998) (73)). However, the basic concepts of the Bayesian Inference (Inverse Modeling) will be described and the DREAM algorithm will be used exclusively for the inference of the rock physics models posterior distributions of parameters - despite its multiple applications. Therefore, during the next sections, firstly, some basic concepts regarding random variables and probability density functions will be presented (Grana (2011) (92)). After this introduction, the basic theory of Bayesian inference with the explanation of the main components needed to compute the posteriors parameters distributions will be also disclosed (Vrugt (2016) (84)). Finally, some metrics of accuracy will be quickly described and defined. In fact, those metrics will be extensively used in the Chapter 6 in order to quantitatively evaluate the performance of the rock physics models of Xu \& Payne and T-Matrix.

\section{1}

\section{Random Variables and Probability Density Functions - PDFs}

It is very important to introduce, before the explanation of the Bayes' Theory itself, some basic concepts regarding random variables and probability density functions. In fact, a quick review about those terminologies is needed because they will be the cornerstone in order to better understand the next section and the Chapters 5 and 6 . Therefore, a variable will be classified as random if its value is somehow uncertain, varying in some order and there is not a specific or a deterministic value that can represent this variable. The following illustration is very useful for a better comprehension of what would be a random variable in comparison with a deterministic value:

- Take into consideration that a rock physics model has been used to calculate the elastic attributes of a carbonate reservoir. Some of the inputs needed for this modeling are the mineral bulk and shear moduli. Thus, the deterministic feeling suggests to assign deterministic values for $\mathrm{K}$ and $\mathrm{G}$ that can be easily obtained from the literature knowing the composition of the reservoir matrix (e.g. 100\% Calcite). However, there are several values of calcite bulk and shear moduli in the literature as can be seen in Table 5.1. Among those values, which would be the right one? 
In case that you want to represent all those values when inserting the inputs in the rock physics modeling, it is possible to generate two continuous random variables $\mathrm{K}$ and $\mathrm{G}$ which will be represented by their respective probability density functions (PDFs). There are several PDFs available in the literature, during this dissertation only the Gaussian and Uniform distributions will be approached. In this way, taking into consideration a continuous random variable $\Theta$, its PDF can be defined as follows:

$$
f_{\Theta}(\theta) d \theta=P(\theta<\Theta \leqslant \theta+d \theta)
$$

Where $f_{\Theta}(\theta)$ is a probability distribution function which computes the probability of any interval of the random variable when integrated, whereas $\mathrm{d} \theta$ is the differential element of infinitesimal length. However, this interval must be in the range: $\theta<\Theta \leqslant \theta+d \theta$. In this way, it is possible to calculate the probability of a set of values in $\Theta$ between the interval $[a, b]$, in the following manner:

$$
P(a<\Theta<b)=\int_{a}^{b} f_{\Theta}(\theta) d \theta
$$

As was already mentioned there are several types of standard continuous and discrete random variables, such as: Gaussian, Beta, Gamma and others. It is out the scope of this dissertation address each one of them, however, the Gaussian distribution which will be used in the following chapters to represent the prior distributions of the rock physics models parameters should be presented (it is important to highlight that in the Bayesian framework we used truncated versions of the original Gaussian distribution). Therefore, the Gaussian distribution is defined as follows:

$$
f_{\Theta}(\theta)=\frac{1}{\sqrt{2 \pi \sigma_{\Theta}^{2}}} \exp \left(-\frac{1}{2} \frac{\left(\theta-\mu_{\Theta}\right)^{2}}{\sigma_{\Theta}^{2}}\right)
$$


Despite the fact that a random variable is represented by its PDF, sometimes, it is possible to define the random variable with some parameters, such as: Mean $\left(\mu_{\Theta}\right)$ and Variance $\left(\sigma_{\Theta}^{2}\right)$. Both of them can be computed in the following manner:

$$
\begin{gathered}
\mu_{\Theta}=\int \theta f_{\Theta}(\theta) d \theta \\
\sigma_{\Theta}^{2}=\int\left(\theta-\mu_{\Theta}\right)^{2} f_{\Theta}(\theta) d \theta
\end{gathered}
$$

In fact, the most probable value that a random variable can be assigned is the mean $\left(\mu_{\Theta}\right)$ whereas the variance $\left(\sigma_{\Theta}^{2}\right)$ indicates how scattered the Gaussian distribution may be. Finally, there is a very useful notation to represent a random variable as a Gaussian distribution that will be widely used during this dissertation:

$$
\Theta \sim \mathcal{N}\left(\mu_{\Theta}, \sigma_{\Theta}^{2}\right)
$$

\section{2 \\ Bayesian Inference}

The Bayesian Inference is grounded in the Bayes' Theorem which is also known as the inverse probability method. Therefore, this approach can be applied in a rock physics inverse problem in the following manner:

$$
\Phi \leftarrow \mathcal{R}(\theta)+\varepsilon
$$

Where $\Phi=\left\{\phi_{1}, \ldots, \phi_{n}\right\}$ is a vector of modeled values provided by the rock physics model, $\theta=\left\{\theta_{1}, \ldots, \theta_{n}\right\}$ a vector of the models parameters and $\varepsilon=\left\{\varepsilon_{1}, \ldots, \varepsilon_{n}\right\}$ is a vector which not only stores observational errors but also errors related to the fact that the rock physics model $\mathcal{R}(\cdot)$ can be different from the reality: $\mathcal{G}(\theta)$ for the parameters $\theta$. Where $\mathcal{G}(\theta)$ is some environmental system, such as: the carbonate reservoir which has been studied 
in this dissertation (Vrugt (2016) (84)). In this way, posing the rock physics inverse problem in a Bayesian framework, it is possible to infer the posterior distribution of parameters $p(\theta \mid \tilde{\Phi})$ taking into consideration the measurements $\tilde{\Phi}=\left\{\tilde{\phi}_{1}, \ldots, \tilde{\phi}_{n}\right\}$, as follows:

$$
p(\theta \mid \tilde{\Phi})=\frac{p(\theta) p(\tilde{\Phi} \mid \theta)}{p(\tilde{\Phi})}
$$

Taking into consideration the following parameters space: $\theta \in \Theta \in \mathbb{R}^{d}$. $p(\theta)$ is the prior distribution of parameters, $L(\theta \mid \tilde{\Phi}) \equiv p(\tilde{\Phi} \mid \theta)$ means the likelihood function and $p(\tilde{\Phi}) \neq 0$ has a normalization property in order to reach a unit computing the integral of $p(\theta \mid \tilde{\Phi})$. In spite of all this, $p(\tilde{\Phi})$ is not required to compute the posterior distribution of parameters through the Bayesian inference. In fact, all statistical inferences related to $p(\theta \mid \tilde{\Phi})$ can be made without normalized terms, thus:

$$
p(\theta \mid \tilde{\Phi}) \propto p(\theta) L(\theta \mid \tilde{\Phi})
$$

The prior parameters distribution $p(\theta)$ can be built based on some prior knowledge about the parameters which will be inputted in the rock physics model. The prior knowledge can be obtained from theoretical information, data previously acquired and interpreted or some qualitative information from the literature (Loures (2001) (21)). As a matter of fact, the prior distribution $p(\theta)$ can be informative (non-flat) or non-informative (flat/uniform). The latter happens when some parameter $\theta$ must be limited in an interval $[a, b]$ and there is not any prior knowledge about the specified parameter, thus, the prior distribution would be described in the following manner:

$$
p(\theta)=\left\{\begin{array}{l}
\frac{1}{b-a} \text { for } a \leqslant \theta \leqslant b, \\
0 \text { for } \theta<a \text { or } \theta>b
\end{array}\right.
$$


A flat distribution leads the posterior parameter distribution vector $p(\theta \mid \tilde{\Phi})$ to be proportional to its likelihood function, as follows:

$$
\begin{gathered}
p(\theta \mid \tilde{\Phi}) \propto p(\theta) L(\theta \mid \tilde{\Phi}) \\
p(\theta \mid \tilde{\Phi}) \propto L(\theta \mid \tilde{\Phi})
\end{gathered}
$$

On the other hand, the prior distribution of the parameters can be informative and non-flat represented by different kind of distributions, such as: Gaussian, Beta, Gamma, Exponential and others. In that case, the posterior parameters distribution will be equivalent to the product of the prior distribution and the likelihood function as it was already described in the Equation 4-9. Thus, it is possible to state the likelihood function represents the summary between the differences of the rock physics model outcomes $\Phi(\theta)$ and the respective observations $\tilde{\Phi}$ from the environmental system $\mathcal{G}(\theta)$. Therefore, assuming the error residuals being independent and normally distributed, then, the likelihood function can be written as:

$$
L(\theta \mid \tilde{\Phi})=\prod_{i=1}^{n} \frac{1}{\sqrt{2 \pi \sigma_{i}^{2}}} \exp \left[-\frac{1}{2}\left(\frac{\tilde{\phi}_{i}-\phi_{i}(\theta)}{\sigma_{i}}\right)^{2}\right]
$$

Where $\sigma_{i}$ and $\sigma_{i}^{2}$ are the standard deviation and variance estimations of the error measurements of the $i$ th observation, respectively. Ultimately, as was already mentioned, for informative prior parameters distributions (nonflat) $p(\theta)$, the posterior parameters distribution $p(\theta \mid \tilde{\Phi})$ is the equivalent of the product between the prior $p(\theta)$ and the likelihood function $L(\theta \mid \tilde{\Phi})$ as can be seen in Equation 4-9. Therefore, sometimes, the posterior parameters distributions can be obtained through analytical manners. However, it is not always possible to accomplish such a task, such as when the models are nonlinear, the posterior distribution $p(\theta \mid \tilde{\Phi})$ can not be reached analytically. In this way, following Scharnagl et al., (2011) (23), in order to solve this problem, it is necessary to use sampling methods to reach a posterior distribution. In fact, 
Markov Chain Monte Carlo simulation, also known as MCMC simulation is the most common of those methods.

It is out of the scope of this chapter describe all the theory behind MCMC simulation and the Metropolis algorithm. There is a wide range of literature approaching those subjects and for a complete and further explanation the following articles are very useful: Brooks et al., (1998) (73), Metropolis et al., 1953 (64) and Vrugt (2016) (84). The latter being the manual of the DREAM algorithm which will be used to run the Bayesian framework in order to evaluate the parameters of the rock physics models described in Chapter 3. In fact, this approach of the DREAM framework shows plenty of advantages in comparison with other techniques, all of them can be analyzed in the work of Vrugt (2016) (84).

\section{3}

\section{Metrics to Measure the Models Accuracy}

One of the main points of this work is to evaluate how accurate both rock physics models approached in this dissertation are regarding elastic attributes estimations for carbonate reservoirs. In this way, it is necessary to use metrics to measure the errors of the models outputs and evaluate how accurate the predictions provided by the models are in comparison with the measurements available. Therefore, the following metrics will be extensively used in Chapter 6 in order to evaluate the accuracy of the rock physics models of $\mathrm{Xu} \&$ Payne and T-Matrix: Mean absolute error (MAE), Root mean squared error (RMSE), Mean error (ME) and Relative error (RE). Each one of them can be defined by the following equations:

Mean Absolute Error:

$$
M A E=\frac{1}{n} \sum_{i=1}^{n}\left|\tilde{\phi}_{i}-\phi_{i}(\theta)\right|
$$

Root Mean Squared Error:

$$
R M S E=\sqrt{\frac{1}{n} \sum_{i=1}^{n}\left(\tilde{\phi}_{i}-\phi_{i}(\theta)\right)^{2}}
$$


Mean Error:

$$
M E=\frac{1}{n} \sum_{i=1}^{n} \frac{\left|\tilde{\phi}_{i}-\phi_{i}(\theta)\right|}{\tilde{\phi}_{i}}
$$

Relative error:

$$
R E=\frac{\tilde{\phi}_{i}-\phi_{i}(\theta)}{\tilde{\phi}_{i}}
$$

Where $n$ is the counter, $\tilde{\phi}_{i}$ represents the measurements and $\phi_{i}(\theta)$ represents the models outcomes. Therefore, the metrics MAE, RMSE and ME are classified as negatively-oriented scores, and this term means that higher values signify low accuracy and lower values better accuracy. Besides that, the relative error ( $\mathrm{RE}$ ) is used to build the histograms between the responses generated through the models and the measurements in Chapter 6 . 


\section{Methodology}

In this chapter the methodology, dataset and tools used during this dissertation will be described. Special attention will be given to the parameters chosen to be stochastically treated and represented by prior distributions in the Bayesian framework. As a matter of fact, not all input parameters of both rock physics models were evaluated and optimized in the DREAM algorithm. The approach of applying the Bayesian framework has the goal of evaluating and optimizing the input parameters of both rock physics models in order to reliably reach the best elastic attributes estimations of the carbonate reservoir possible. However, taking into consideration the uncertainties related to the input parameters, it is very important to highlight that some caution is needed regarding the choice of the prior distributions which will be inputted in the Bayesian framework. In this way, the foundations of each prior parameter distribution will be explained, supported and justified following all the geological information in the dataset and also in the literature. Besides that, during this chapter, in section 5.2, not only the stochastic inputs addressed in the Bayesian framework will be explained but also the deterministic ones which were not evaluated stochastically. Furthermore, in section 5.1, it will be possible to describe the tools used in this dissertation and also understand the challenges faced regarding the dataset used, such as: Quality control of the well logs and the thin sections images.

\section{1}

\section{Dataset, Data Quality Control and Tools}

\subsection{1}

\section{Quality Control of Well Logs and Core Samples Measurements}

Three well $\log$ suites from three different wells were available for this dissertation. Most of them are composed by the following well logs: DT (Sonic), GR (Gamma Ray), RHOB (Bulk Density), NPHI (Neutron Porosity), PHIT (Total Porosity), PHIE (Effective Porosity), $S_{w}$ (Water Saturation), CALI (Caliper), $V_{s h}$ (Shale Content) and ILD (Resistivity). None of those well logs suites had the DTS well log which is related to the $\mathrm{S}$ wave velocity as well as 
the DT $\log$ (Sonic) which is related to the $\mathrm{P}$ wave velocity. In fact, the basic well log suites are composed by NPHI, DT, ILD, RHOB, GR and CALI. Other well logs, such as: $V_{s h}$, PHIE and $S_{w}$ are generated through the logs inside the basic suite. In the same way, 22 thin sections images were used to make the pores aspect ratios measurements with their respective volume fractions, all of them were extracted from the work of Bizotto (2014) (15).

Regarding well log analyses, the first challenge was to separate the coquinas from the mudrocks and shales. In fact, it is possible to notice in the work of Bizotto (2014) (15) that is very common to observe that coquinas reservoirs are interleaved with mudrocks and shales. Therefore, the mudrocks and shales were cut off from the well logs. Moreover, another important well log that supported the analysis and delimitation of coquinas was the caliper $\log$ (CALI). In short, the caliper log measures the diameter of the well hole and through the analysis of those measurements it is possible to try to identify well washouts or even anomalous values of the hole diameter due to drilling. Several times high values of caliper were discovered in the well log suites, indicating washouts and each one of those measurements and the related well logs measurements at the same depth, inside of the well log suites, were cutted off.

It is also important to mention that, besides the caliper cutoff, it was also necessary to make the cutoff related to the GR and $V_{s h} \operatorname{logs}$. In fact, plenty of times it was very easy to identify and cut high values of $V_{s h}$ because the shale was really interleaved with the coquinas. However, a criteria was established to cut even the dirty coquinas and this criteria was generated with the well $\operatorname{logs}$ of GR and $V_{s h}$. Therefore, after all those changes, it was possible to obtain the coquinas as clean as possible. Nevertheless, it is also important to highlight that besides the well logs, 14 sets of dry $V_{p}$ and $V_{s}$ lab measurements of core samples were available for this work. These core samples were extracted from different wells allocated in the same coquinas reservoir. Moreover, all of them have the same facies of the carbonate reservoir profiled by the well logs but most of the core samples did not come from the three wells analyzed in this dissertation.

\section{1 .2 \\ Quality Control of the Thin Sections Images and Aspect Ratio Measure- ments}

In the matter of pores aspect ratio measurements, other challenges were faced. As mentioned before, all the thin sections images used were taken from 
the work of Bizotto (2014) (15). In this way, 22 thin sections images were used among all images available in the dissertation. The reason why just 22 images were chosen, is because most of the time, the thin sections images did not have pores or the image quality to measure the aspect ratio was too poor. Moreover, the software used to make the pores aspect ratios measurements was the ImageJ software (https://imagej.nih.gov/ij/). ImageJ is a software of image processing and analysis developed in Java which was extremely helpful in order to measure the pores aspect ratios from those images (Ferreira and Hasband, (2012) (71)). Besides that, due to the low resolution of the thin sections images, it was not possible to make an automatic extraction of the pores aspect ratios. Therefore, a meticulous work was necessary to manually measure each aspect ratio in all thin sections images totalizing 105 pores aspect ratios measurements in the 22 thin sections images available. The procedure to measure the aspect ratios was pretty simple and can be found in a very didactic manner in the ImageJ's manual (just take into account that the measurements were made through ellipses fitting in the pores of the thin sections images). For illustration purposes, in Figure 5.1, there are some of the thin sections images chosen to make the aspect ratio measurements. Besides that, in Appendix A, some examples of the fitted ellipses in the thin section images are also shown.

\subsection{3}

\section{Deploys of the Rock Physics Models and the DREAM Framework}

It is important to highlight that the rock physics models of Xu and Payne and the T-Matrix approach were deployed with Python and/or Matlab and all data manipulation in the well log suites in section 5.1.1 was made with the Pandas Package in Python. In fact, the deploys of both rock physics models were validated with the respective articles from the literature reaching the same results obtained by the authors in the following articles: $\mathrm{Xu}$ and Payne (2009) (86), Jakobsen et al., (2003a,b) (54, 53). Moreover, the settlement of the rock physics models parameters in the Bayesian framework was achieved through a MATLAB toolbox that is based in the Markov chain Monte carlo (MCMC) simulation and the DiffeRential Evolution Adaptive Metropolis (DREAM) algorithm which has been used in several research fields and was developed by Vrugt et al., (2008a, 2009a) (35, 33). 


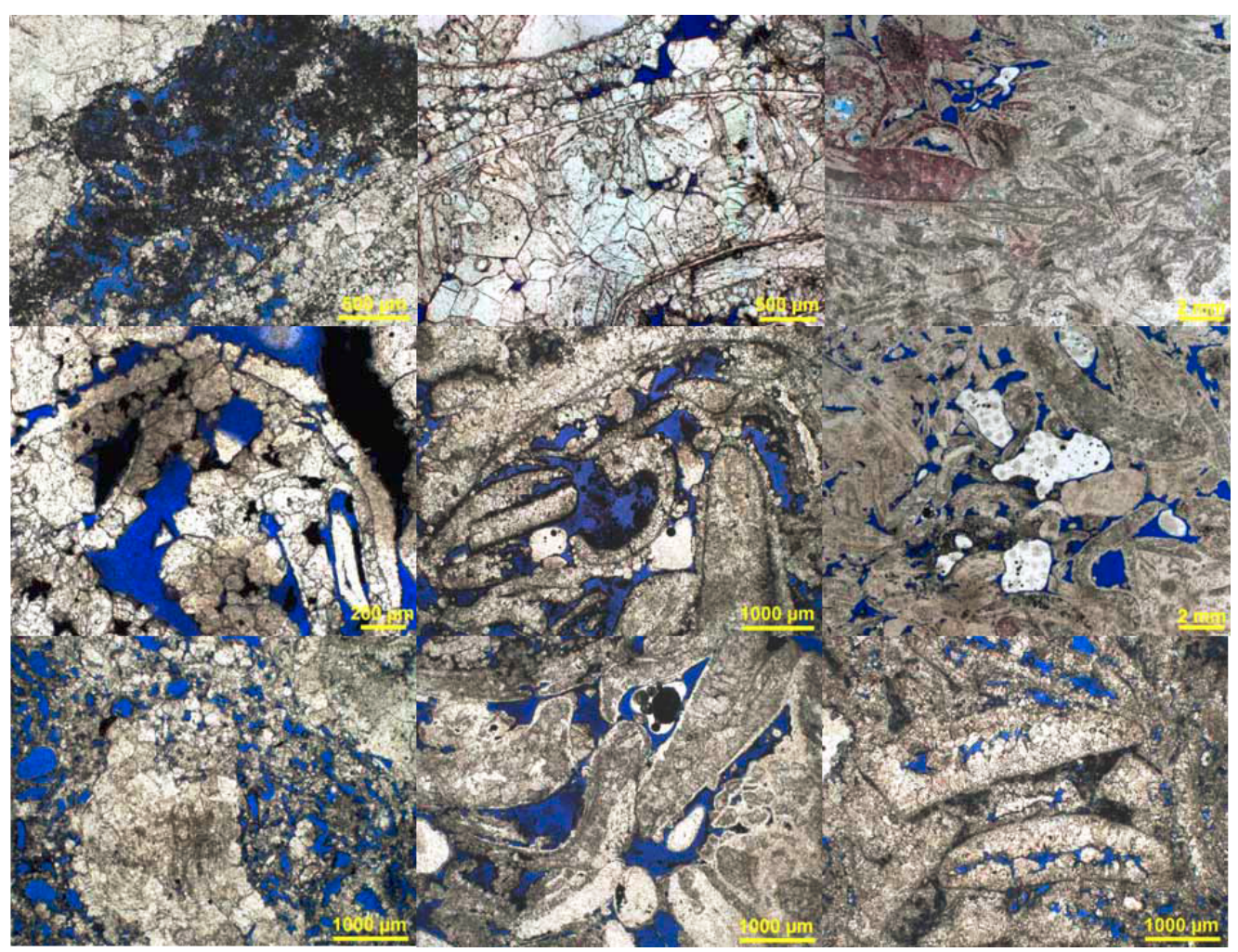

Figure 5.1: Some of the thin sections images used to make the pores aspect ratios measurements where the blue color represents the pore space. Image from Bizotto (2014) (15).

\section{2 \\ Stochastic and Deterministic Parameters}

After the explanation regarding the dataset and tools used in this dissertation, it is necessary to explain and describe the inputs used in each rock physics model and why some of them were stochastically treated in the Bayesian framework. In fact, some parameters are common for both models. However, as it was possible to notice in Chapter 3, the T-Matrix approach is a frequecy-dependent model whereas the $\mathrm{Xu} \&$ Payne only works for low frequencies because of the Gassmann's relations. Therefore, the following parameters are common for both models: mineral bulk and shear moduli $(\mathrm{K} \mid \mathrm{G})$, density $(\rho)$, aspect ratios $\left(\alpha_{1} \mid \alpha_{2}\right)$, volume proportions $\left(V_{1} \mid V_{2}\right)$ and fluids bulk modulus and density $\left(K_{f l} \mid \rho_{f l}\right)$. On the other hand, the parameters: relaxation time constant $(\tau)$, frequency $(\omega)$ and connectivity $(\beta)$ are exclusively used in the T-Matrix approach. Despite all this, only the parameters related to the mineral bulk and shear moduli $(\mathrm{K} \mid \mathrm{G})$, aspect ratios $\left(\alpha_{1} \mid \alpha_{2}\right)$ and volume proportion $\left(V_{1}\right)$ will be stochastically addressed and represented by probability density functions (PDFs) in the Bayesian framework whereas the other parameters will be deterministically inputted. 


\subsection{1}

\section{Stochastic Parameters}

\subsubsection{1}

\section{Mineralogical Content - Bulk and Shear Elastic Moduli}

In the rock physics point of view, the reservoir rock is composed of three entities: matrix, pore network and fluids. As it was possible to observe in the section 3.1.1, the matrix can be modeled as an elastic solid media if the following information is available: Volume fractions of the mineralogical content which compose the matrix and their respective elastic moduli (mineral bulk and shear moduli). Generally, the mineralogical content can be obtained through X-Ray Difraction technique, also known as XRD analysis. In short, the XRD analysis compute the mineralogical content of core samples through a small sample of the core itself. Part of the dataset available for this work was composed by core samples and their respective XRD measurements but no information regarding the mineral bulk and shear moduli was provided.

The XRD measurements available for this work pointed out that the coquinas core samples are composed predominantly by the mineral calcite with other much less important contents of quartz and dolomite. Therefore, it was considered in the rock physics modeling that the reservoir is composed of $100 \%$ calcite. However, without the information about the mineral bulk and shear moduli, which kind of values $K_{o}$ and $G_{o}$ should be used to represent the reservoir matrix in the rock physics modeling? Table 5.1 shows some values of bulk and shear moduli of the minerals calcite and dolomite. This table was extracted from Mavko et al., (2009) (68) and all those values were extracted from several different references that can be further analyzed in their books.

In Table 5.1, it is possible to notice a wider range of the calcite bulk modulus, a lower range of the shear modulus and the density values are almost constant for different values of $\mathrm{K}$ and $\mathrm{G}$. Moreover, some values of the calcite bulk modulus are almost equal to some of the values of the dolomite bulk modulus whereas the dolomite shear modulus shows much higher values in comparison to the calcite shear modulus values. Therefore, it would not be fair to choose only one of those bulk and shear moduli as input in the rock physics models and assume that would be the mineral bulk and shear moduli and consequently, the matrix elastic moduli. 
Table 5.1: Values of Shear and Bulk moduli of Calcite and Dolomite Minerals.

\begin{tabular}{lccc} 
Mineral & Bulk Modulus (GPa) & Shear Modulus $(\mathbf{G P a})$ & Density $\left(\mathbf{g} / \mathbf{c m}^{\mathbf{3}}\right)$ \\
\hline \multirow{2}{*}{ Calcite } & 76,8 & 32 & 2,71 \\
& 63,7 & 31,7 & 2,70 \\
& 70,2 & 29 & 2,71 \\
& 74,8 & 30,6 & 2,71 \\
& 68,3 & 28,4 & 2,71 \\
Dolomite & 94,9 & 45 & 2,87 \\
& 69,4 & 51,6 & 2,88 \\
& 76,4 & 49,7 & 2,87
\end{tabular}

Table from Mavko et al., (2009) (68).

After analyzing the previous table, it is possible to conclude that deterministic values could not be the best option to represent $K$ and $G$ as inputs for the rock physics models described in Chapter 3. Facing this uncertainty issue and taking into consideration this quantitative information from the literature, it is very suggestive to represent both $\mathrm{K}$ and $\mathrm{G}$ by normal Gaussian distributions that will be part of the prior parameters distributions vector $p(\theta)$ in the Bayesian framework used in this dissertation through the DREAM algorithm. Those parameters distributions are mathematically expressed in the following manner: $K \sim \mathcal{N}\left(\mu_{K}, \sigma_{K}^{2}\right)$ and $G \sim \mathcal{N}\left(\mu_{G}, \sigma_{G}^{2}\right)$ where $\mu_{G}, \mu_{K}$, $\sigma_{G}^{2}$ and $\sigma_{K}^{2}$ are the distributions means and variances, respectively. Therefore, Figures 5.2 and 5.3 illustrate both Gaussian distributions.

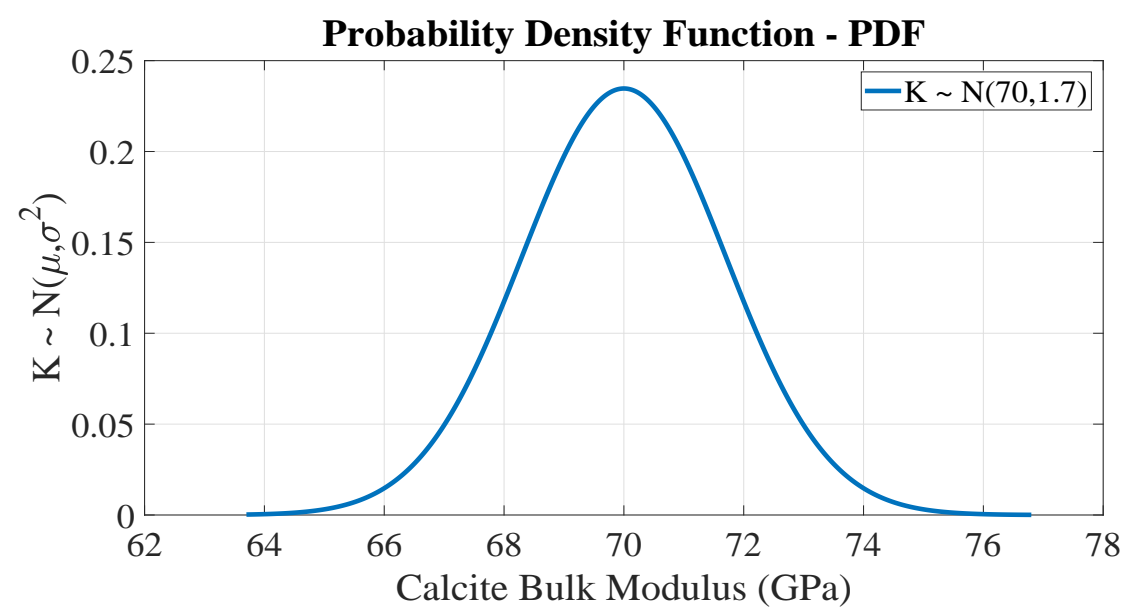

Figure 5.2: Gaussian distribution representing the calcite elastic bulk modulus. 


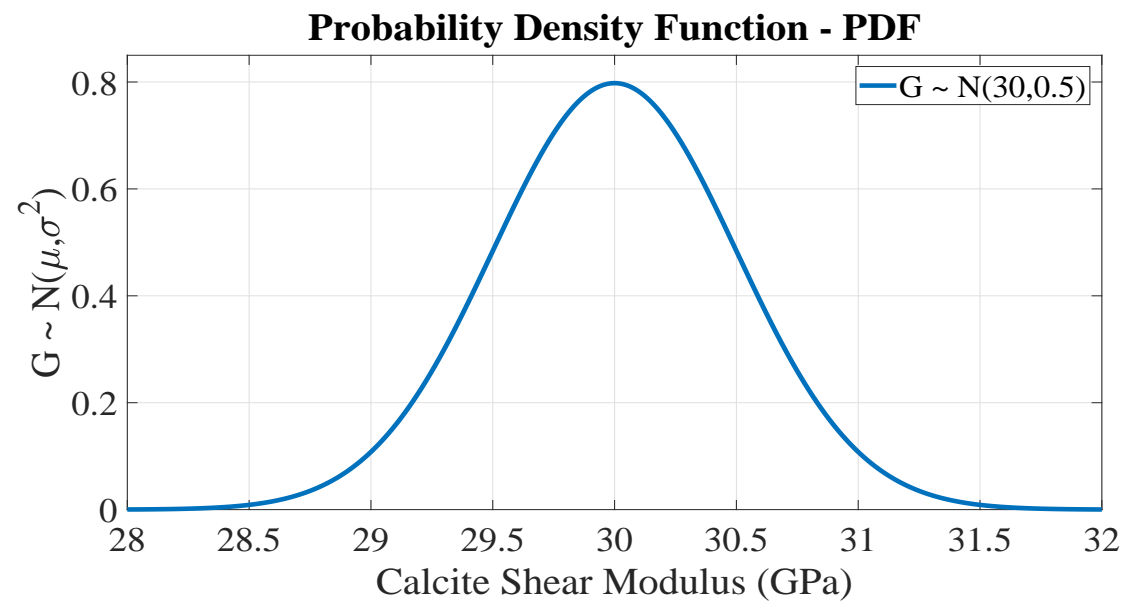

Figure 5.3: Gaussian distribution representing the calcite elastic shear modulus.

In Figures 5.2 and 5.3, it is possible to notice that the Gaussian distributions which represent the calcite elastic moduli have the following values of mean and variance:

$$
\begin{aligned}
& K \sim \mathcal{N}\left(\mu_{K}=70, \sigma_{G}^{2}=1.7\right) \\
& G \sim \mathcal{N}\left(\mu_{G}=30, \sigma_{G}^{2}=0.5\right)
\end{aligned}
$$

Besides that, those distributions are constrained by the following bounds:

$$
K \sim[63.7,76.8]
$$

$$
G \sim[28,32]
$$

The criteria used to build both distributions was Table 5.1 where the mean values $\left(\mu_{K}\right.$ and $\mu_{G}$ ) of the distributions $\mathrm{K}$ and $\mathrm{G}$ are the arithmetic mean of calcite elastic moduli values ( $K$ and $G$ ) in table 5.1. In the same way, the bounds were generated following the same table where $\mathrm{K}$ and $\mathrm{G}$ vary in the following manner approximately: $63.7 \leqslant K \leqslant 76.8$ and $28 \leqslant G \leqslant 32$. The variance values were settled to better distribute the Gaussian along the $\mathrm{x}$-axis giving the correct density of probability for each value of $\mathrm{K}$ and $\mathrm{G}$. In this 
way, despite the fact that no information of in situ calcite $\mathrm{K}$ and $\mathrm{G}$ of the carbonate reservoir was available, it was possible to build the prior parameters distributions taking into consideration information from the literature.

Finally, the reservoir rock density was not approached as a stochastic parameter because of the low variation of calcite density in Table 5.1. Thus, the following value of density in the Bayesian framework using both rock physics models was assumed: $\rho=2.710 \mathrm{~kg} / \mathrm{m}^{3}$.

\subsubsection{2}

\section{Pores Aspect Ratios and their Volume Proportions}

Since the prior parameters distributions of the calcite bulk and shear moduli were already settled with a geological reasoning, the next step is to make the same analysis for inputs related to the pores network that should be generated in the elastic solid matrix. Therefore, regarding the pores network modeling, two main parameters should be highlighted: the pores aspect ratios and their volume proportions. Thus, to obtain these parameters, it was necessary to make the pores aspect ratios measurements as can be seen in section 5.1.2, where 105 aspect ratios were measured in 22 thin sections images. Figure 5.4 shows a histogram that gives some insights about the volume distribution of the measured aspect ratios. Moreover, the vertical axis represents the volume percentage of each aspect ratio measured in all the thin section images and the horizontal axis represents each value of aspect ratio.

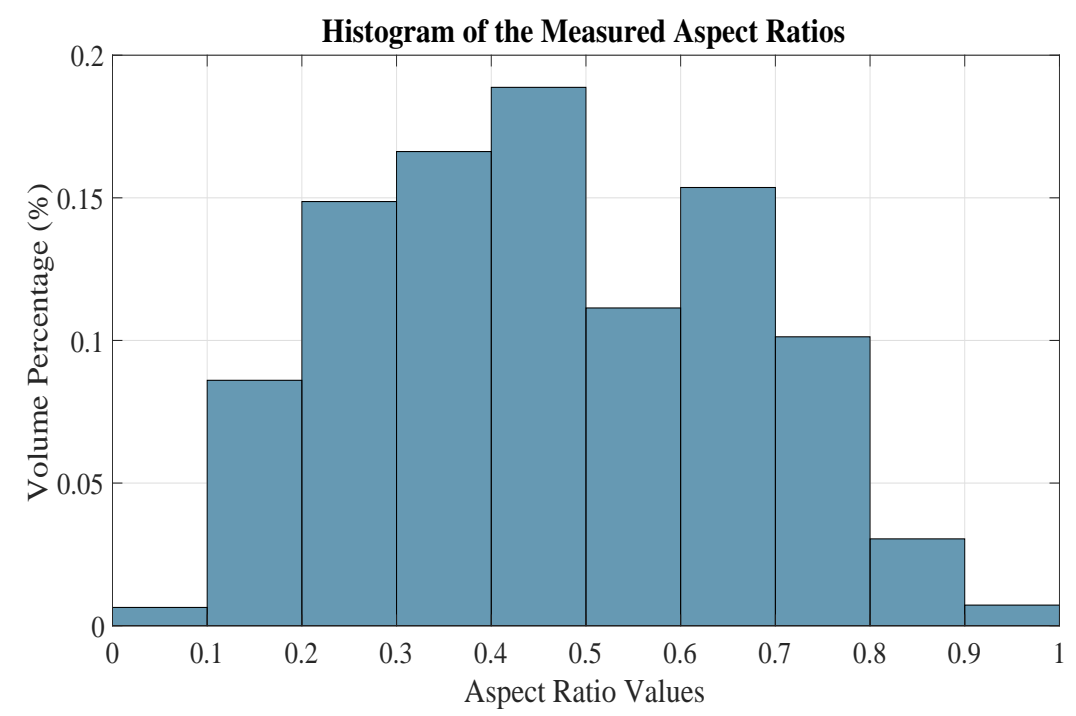

Figure 5.4: Histogram of all aspect ratio measurements in the 22 thin sections normalized by the volume percentage. 
Figure 5.4 shows the histogram of all aspect ratios measured in the thin sections images available and it is possible to conclude that there is a greater volume percentage of pores in the following range of aspect ratios: $0.2 \leqslant \alpha<0.5$. Besides that, it is also possible to notice that pores with low $(\alpha<0.1)$ and high values $(0.8 \leqslant \alpha \leqslant 1)$ of aspect ratio tend to present lower volume percentage in the measurements made in the thin sections of the coquinas reservoir. Therefore, the following ranges of aspect ratios: $\alpha<0.1$ and $\alpha \geqslant 0.9$ just represent approximately $1 \%$ of the total volume of aspect ratios measured. In fact, only one pore was discovered with $\alpha \geqslant 0.9$ and four pores $\alpha<0.1$. Nevertheless, it should be important to highlight that there is a kind of pore type which is very difficult to see through human eyes in thin sections: crack pores. It is possible to notice in Figure 5.4 that the predominant volume percentage represented by the pores aspect ratios varies approximately in the following range: $0.1 \leqslant \alpha<0.9$. In this way, crack pores with aspect ratios around 0.001 and 0.01 are not likely to be seen/observed and there is a lack of literature which approaches the crack pores percentage in carbonate rocks, especially in coquinas reservoirs.

It would not be right to neglect the presence of crack pores in carbonates due to the intrinsic carbonates brittle nature already approached in Chapter 2. As a matter of fact, crack pores in the rock physics models can be represented by grain-to-grain contacts, microporosity and microfractures. Besides that, some people believe that cracks aspect ratios and their volume proportions are fitting parameters in rock physics modelings. Therefore, taking into consideration the previous premises and the fact that there is a lack of literature addressing this subject, it is possible to make a kind of approximation and fairly cogitate that this kind of porosity can represent until $10 \%$ of the reservoir volume, a kind of porosity which is not likely to be seen/observed in thin sections because of very low aspect ratios presented $(0.001 \leqslant \alpha \leqslant 0.01)$.

After analyze the most likely volume proportions of cracks in the carbonate reservoir, it is necessary to disclose about the possible values of aspect ratios that this kind of pore can assume. However, there is not a wide variety of literature approaching aspect ratios of crack pores for different types of carbonate rocks. On the other hand, Xu and Payne (2009) (86) used the following values to represent the aspect ratios of interparticle pores, crack pores and moldic pores, respectively: $\alpha_{\text {interparticle }}=0.15, \alpha_{\text {cracks }}=0.02$ and $\alpha_{\text {moldic }}=$ 0.8. Besides that, using carbonates core samples from the University of Miami database, they ran the pore-type inversion workflow of Kumar and Han (2005) (59) and found out that the range of crack porosity present in those core samples varied approximately in the following manner: $0.002 \leqslant \alpha_{\text {cracks }} \leqslant 0.018$. 
Moreover, following Xu \& Payne (2009) (86), it is possible to state that the pore volume of a carbonate rock can be divided in the following manner:

$$
V_{T}=V_{\text {stiff }}+V_{\text {interparticle }}+V_{\text {cracks }}
$$

Where the $V_{T}$ is the total pore volume of the carbonate reservoir, $V_{\text {cracks }}$ is the volume of cracks pores, $V_{\text {stiff }}$ is the volume of moldic pores and $V_{\text {interparticle }}$ represents the volume of interparticle pores. Despite all that, it is difficult to deterministically state a volume proportion for each pore type in a carbonate reservoir. There is no direct way to obtain this quantitative information in a reliable manner without taking into consideration uncertainties. Therefore, after this brief discussion and grounded on those premises regarding the pores aspect ratios and their volume proportions, it was possible to verify the need to approach those parameters stochastically through the Bayesian inference, in order to achieve a better parameters evaluation taking into account the intrinsic uncertainties related to the aspect ratios and their volume proportions. In fact, regarding our specific case, the total pore volume $V_{T}$ of the carbonate reservoir which has been studied will be divided in two volumes of pore types, as follows:

$$
V_{T}=V_{1}+V_{2}
$$

Where $V_{1}$ and $V_{2}$ represent the volume of crack pores and the volume of non-crack pores, respectively. In fact, the volume of non-crack pores represents the volume of the measured pores obtained through the measurements in the thin sections images available. Thus, the volume of the non-crack pores is computed in the following manner:

$$
V_{2}=1-V_{1}
$$


In this way, the volume of crack pores in the carbonate reservoir $\left(V_{1}\right)$ will be computed as a prior parameter distribution which will be represented by a uniform distribution as can be illustrated in Figure 5.5.

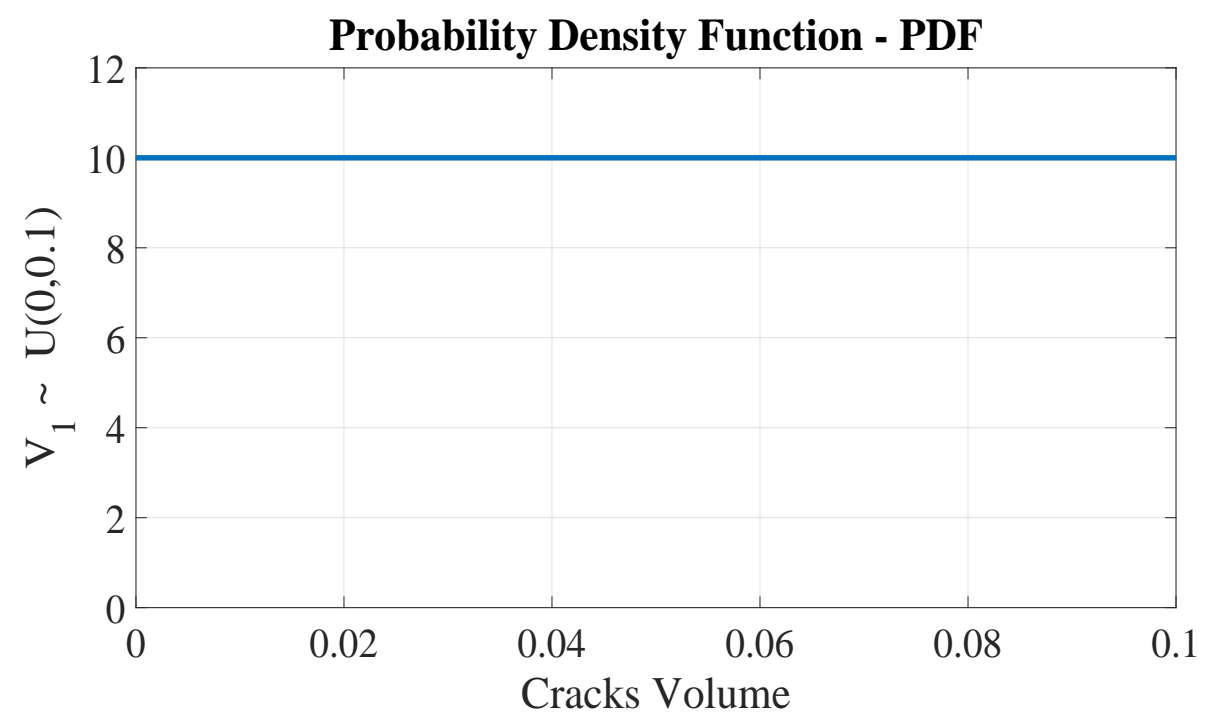

Figure 5.5: Gaussian Distribution Representing the Volume of Crack Pores.

The distribution presented in Figure 5.5 is mathematically represented in the following manner:

$$
V_{1} \sim \mathcal{U}(0,0.1)
$$

Thus, it is constrained by the following bounds:

$$
V_{1} \sim[0,0.1]
$$

The criteria used to build this uniform distribution as a prior parameter was the premise that the coquinas reservoir can have until $10 \%$ of the volume proportion equals to cracks pores. Taking into consideration the lack of literature regarding this subject for carbonates, mainly for coquinas, we took into consideration a uniform distribution because there is not any prior knowledge regarding this parameter where all the values have the same density of probability. In the same way that the carbonate reservoir will be modeled with two different volumes of pore types - cracks $\left(V_{1}\right)$ and non-cracks $\left(V_{2}\right)$ -, as was already described, it is also necessary to stochastically approach 
the aspect ratios which will be represented by those two volume fractions. Therefore, Figures 5.6 and 5.7 illustrate both distributions of aspect ratios $\left(\alpha_{1}\right.$ and $\left.\alpha_{2}\right)$ that will be used as inputs of the parameters evaluation through the Bayesian Inference using the DREAM algorithm. Take into consideration that $\alpha_{1}$ is related to the aspect ratios of the crack pores whereas $\alpha_{2}$ is related to the aspect ratio of the measured pores in the thin section images.

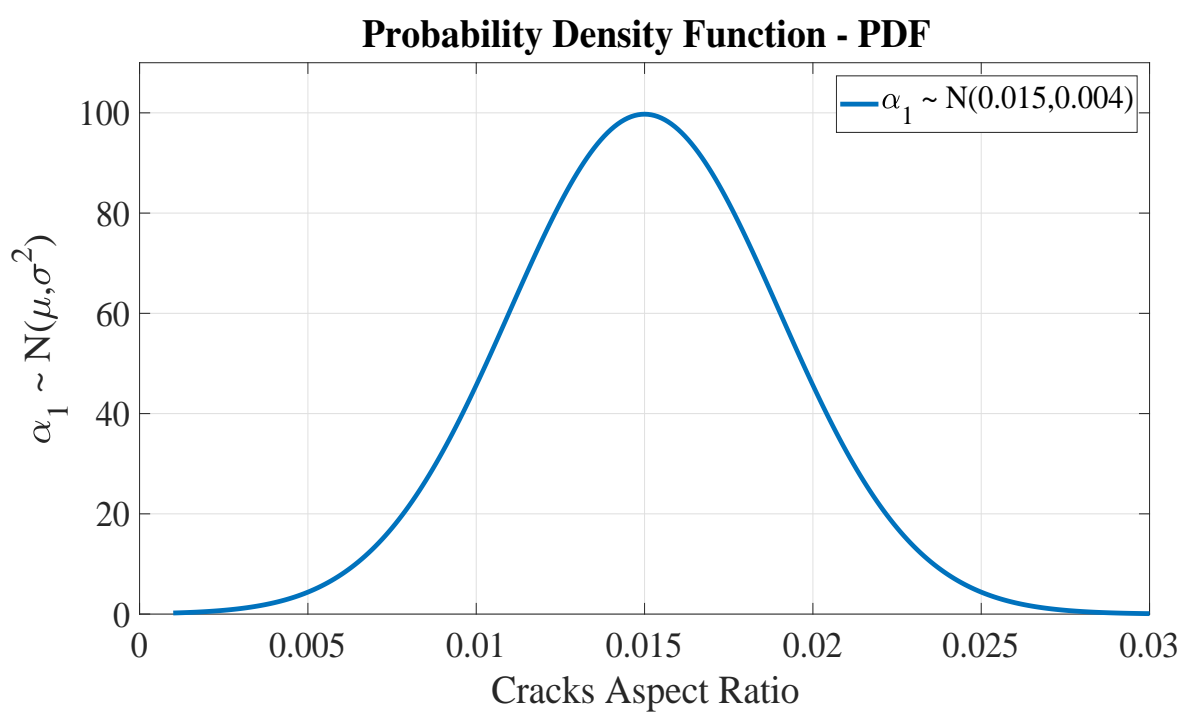

Figure 5.6: Gaussian distribution representing the values of aspect ratio of the crack pores.

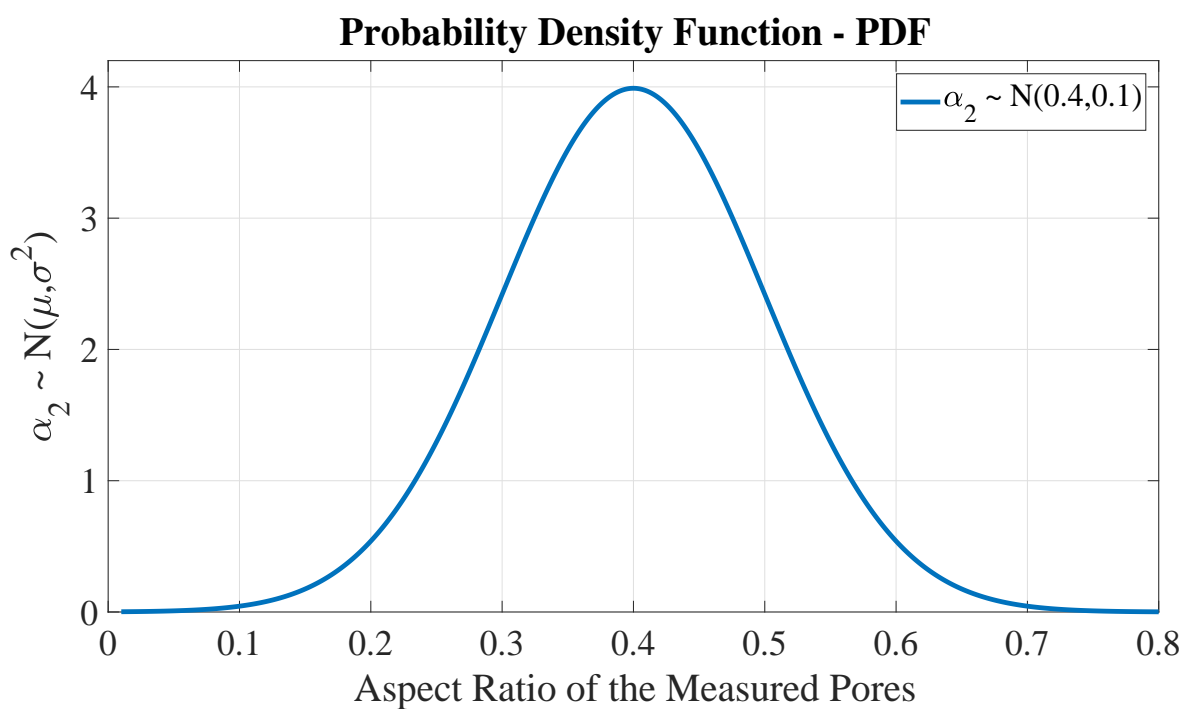

Figure 5.7: Gaussian distribution representing the values of aspect ratio measured in the thin sections. 
The Gaussian distribution of Figure 5.6 represents the crack pores aspect ratios and has the following properties:

$$
\alpha_{1} \sim \mathcal{N}\left(\mu_{\alpha_{1}}=0.015, \sigma_{\alpha_{1}}^{2}=0.004\right)
$$

Moreover, this distribution is constrained by the following bounds:

$$
\alpha_{1} \sim[0.001,0.03]
$$

Likewise, the Gaussian distribution of Figure 5.7 represents the measured pores aspect ratios and has the following properties:

$$
\alpha_{2} \sim \mathcal{N}\left(\mu_{\alpha_{2}}=0.4, \sigma_{\alpha_{2}}^{2}=0.1\right)
$$

Moreover, this distribution is constrained by the following bounds:

$$
\alpha_{2} \sim[0.01,0.8]
$$

The criteria used to build the Gaussian distribution $\alpha_{1}$ was the outcome obtained in the article of Xu \& Payne (2009) (86), where a pore-type inversion workflow was conducted in carbonate core samples and the following range of crack pores was obtained: $0.002 \leqslant \alpha_{\text {cracks }} \leqslant 0.018$. In order to take into consideration a wider range of cracks values in our parameters evaluation through the Bayesian framework, the following range was taken into consideration (bounds): $0.001 \leqslant \alpha_{\text {cracks }} \leqslant 0.03$.

The Gaussian distribution $\alpha_{2}$ was built grounded in the histogram of Figure 5.4. Therefore, in order to build a reliable and representative Gaussian distribution of this histogram, the high volume percentage inside the range of $0.6 \leqslant \alpha<0.7$ was not considered. On the other hand, the high volume percentages of the range $0.2 \leqslant \alpha<0.6$ was honored as well as the low volumes $\alpha \leqslant 0.1$ and $\alpha \geqslant 0.8$. This approximation was needed because it is not possible to take into account high values of density of probabilities 
for the range $0.6 \leqslant \alpha<0.7$ which represents only $15 \%$ of the pores volume percentage without a strong decrease in the density of probabilities of the range $0.2 \leqslant \alpha<0.6$ which represents more than $60 \%$ of the pores volume. In this way, it was preferable to make the PDF centered in $\alpha=0.4$ and honor almost all the histogram volume percentages with the respective densities of probabilities than try to honor the volume percentage in the range $0.6 \leqslant \alpha<$ 0.7 and misrepresent all the other volumes. Besides that, in this PDF, the following interval of aspect ratio was taken into consideration (bounds): [0.01, 0.8]. This interval represents more than $96 \%$ of the pores volume percentage.

\section{2 .2 \\ Deterministic Parameters}

\subsubsection{1}

\section{Fluids}

Regarding the fluids information, in our case, it was not evaluated as a stochastic input in the Bayesian framework through the DREAM algorithm. It does not signify that we truly believe that all information regarding the fluids do not embed uncertainties. In fact, the fluids inputs are: the water saturation well $\log \left(S_{w}\right)$, which is obtained from the resistivity log (ILD), and also the reservoir average fluids in situ information: averaged bulk modulus $\left(K_{f l}\right)$ and averaged density $\left(\rho_{f l}\right)$. Despite all that, all of them will be treated deterministically in the Bayesian framework and also in the sensitivity analyses in Chapter 6. Therefore, this section goal is to quickly summarize how the fluids information are inputted in both rock physics models and how the water saturation log, and the averaged fluids bulk modulus and density are used together to simulate fluids mixture in the carbonate reservoir.

The rock physics models of $\mathrm{Xu} \&$ Payne and T-Matrix work in different perspectives regarding fluids inclusions and/or interactions. In Chapter 3, it was possible to notice that the $\mathrm{Xu} \&$ Payne rock physics model uses the Gassmann's fluid substitution to saturate the dry elastic matrix in non-crack pores (interparticle and moldic) whereas the T-Matrix approach uses the inclusion based theory to insert fluids in all the pores present in the dry elastic solid matrix. Regarding the Gassmann's fluid substitution used in the $\mathrm{Xu} \&$ Payne approach, it is necessary to take caution because of the many assumptions embedded in the Gassmann's relations (Mavko et al., (2009) (68)). One of them that should be highlighted is that the $\mathrm{Xu} \&$ Payne rock physics model should work strictly for low frequencies because one of the Gassmann's assumptions is that the wave induced pore pressure must dissipate 
in a seismic period. On the other hand, the T-Matrix rock physics model is a frequency-dependent model and the frequency is an input parameter that must be settled in the T-Matrix. In this way, it is possible to simulate, in the TMatrix, different frequencies from low frequencies (seismic) to high frequencies (lab measurements). In fact, in the next section, some parameters which are exclusively of the T-Matrix rock physics model will be approached, not only the frequency $(\omega)$ but also the connectivity $(\beta)$ and the relaxation time constant $(\tau)$.

Most of the time, in a reservoir column profiled by well log measurements, it is very frequent the presence of more than one type of fluid. In this case, for more accurate elastic attributes estimations of the rock physics model, it is necessary to compute the bulk modulus and density of the fluids mixture present in each sample of the well log. In order to accomplish such a task, it is necessary to take the proportion of each kind of fluid throughout the reservoir column using the water saturation $\log \left(S_{w}\right)$. In this way, the oil saturation can be obtained through the following relation - taking into consideration a two phases system $\left(S_{o}\right)$ :

$$
S_{o}=1-S_{w}
$$

Thus, it is standard procedure to use the Reuss' Average (76) to calculate the bulk modulus of a fluid mixture, as described in section 3.1.3.1.

$$
\frac{1}{K_{f l}}=\sum_{i=1}^{N} \frac{f_{i}}{K_{i}}
$$

Therefore, taking into consideration the presence of brine $\left(f_{\text {brine }}\right)$ and oil $\left(f_{\text {oil }}\right), K_{f l}$ can be obtained, as follows:

$$
K_{f l}=\frac{1}{\sum_{i=1}^{N} \frac{f_{i}}{K_{i}}}=\frac{1}{\frac{f_{\text {oil }}}{K_{\text {oil }}}+\frac{f_{\text {brine }}}{K_{\text {brine }}}}
$$

After the computation of $K_{f l}$, it is necessary to obtain the density of the fluids mixture for each sample of the profiled reservoir. In order to achieve 
this, the $\rho_{f l}$ can be calculated through the Voigt's Average (83) as can be seen in section 3.1.3.1:

$$
\rho_{f l}=\sum_{i=1}^{N} f_{i} \rho_{i}
$$

Then:

$$
\rho_{f l}=f_{\text {oil }} * \rho_{\text {oil }}+f_{\text {brine }} * \rho_{\text {brine }}
$$

This is the standard procedure for both rock physics models to obtain the values of bulk modulus and density of the fluid mixture present in each sample of the well log.

\subsubsection{2}

\section{Frequency, Relaxation Time Constant and Connectivity}

In the last section of this Chapter some parameters will be discussed, which are exclusive of the T-Matrix rock physics model, and the implications to set each one of them in the right manner will also be discussed. It is important to mention that they were also treated deterministically in the Bayesian framework and also in the sensitivity analyses in Chapter 6. In this way, taking into consideration that the T-Matrix is a frequency-dependent rock physics model, it is possible to work with different values of frequency as inputs in the T-Matrix. Therefore, it is possible to simulate not only seismic frequencies (low frequencies) which can vary between $0.1 \mathrm{~Hz}$ to $50 \mathrm{~Hz}$ approximately but also well $\log$ and laboratory frequencies, which are, approximately, in the following ranges, respectively: $1 \mathrm{kHz}$ to $100 \mathrm{kHz}$ and $100 \mathrm{kHz}$ to $10 \mathrm{MHz}$.

During all the rock physics modelings generated through the T-Matrix rock physics model in this dissertation, only low frequencies were used as inputs, generally the frequency parameter assumed the following value: $\omega=10$ Hz. In fact, the main reason to estimate the elastic attributes through the TMatrix model in a low frequency perspective is to make a fair comparison with the $\mathrm{Xu} \&$ Payne model. As it was already explained, the Xu \& Payne model only estimates elastic attributes in a low frequency perspective because the 
Gassmann's relations are embedded in the model workflow. Thus, in order to evaluate which model has a better performance regarding the elastic attributes estimations of coquinas, both of them will estimate the attributes in a low frequency perspective.

The other input which is exclusive of the T-Matrix approach due to the fact that this rock physics model takes into consideration the squirt flow effect is the relaxation time constant. In Chapter 3, a quick review about this subject was already addressed and it was shown how many parameters are needed to obtain the relaxation time constant. In fact, Jakobsen et al., (2003b) (53) stated that a lot of uncertainties can be discovered related to the relaxation constant $(\tau)$. In this way, taking into consideration that the elastic attributes estimations using the T-Matrix were computed only for low frequencies, the relaxation time constant used was the standard value of $\tau=10^{-5}$ extracted from the article of Jakobsen et al., (2003b) (53).

Last but not least, the connectivity $(\beta)$ input, it is a binary parameter which can only assume two values: 1 or 0 . In fact, in the T-Matrix rock physics model, it is possible to connect or disconnect the families of pores inside of the elastic solid media. In this way, when $\beta=1$ all the pores embedded in the same family are connected whereas when $\beta=0$, the pores are disconnected from each other. This parameter is also very important because when all the communications between the possible families of pore shapes in the elastic solid media are closed, it is possible to simulate high frequency elastic attributes estimations because the wave induced pore pressure will not dissipate within in a seismic period. On the other hand, when all the pores families are connected, a low frequency model is simulated, and the rock physics model will generate low frequency elastic attributes estimations. In all cases during this dissertation, using the T-Matrix rock physics model, the two families of pore shapes $\alpha_{1}$ and $\alpha_{2}$ are connected, inclusive, in the parameters evaluation through the Bayesian framework and in the sensitivity analyses. 


\section{6 \\ Results and Discussions}

In this chapter, all the outcomes obtained through this dissertation will be described and discussed. All the information given in the previous chapters during the dissertation will be used to run the parameters evaluation using the Bayesian framework, the elastic attributes estimations and comparisons through both rock physics models and the sensitivity analyses. It was possible to achieve those tasks executing the following steps:

- The parameters related to the generation of the elastic solid media ( $\mathrm{K}$ and $\mathrm{G}$ ) as well as the parameters related to the pore network (V1, $\alpha_{1}$ and $\alpha_{2}$ ) were posed in a Bayesian framework in order to evaluate and optimize those parameters using the DREAM algorithm.

- The maximum à posteriori (MAP) - deterministic values which better represent the posterior parameters distributions - values were taken from the posteriors parameters distributions generated through the Bayesian framework and applied in both rock physics models. Therefore, it was possible to run the rock physics models parametrization and calibration at the well location (Well 49). Besides that, with both models calibrated, it was also possible to validate if both of them had a good power of extrapolation estimating elastic attributes for other wells. Furthermore, the elastic attributes estimations of $\mathrm{Xu} \&$ Payne and T-Matrix were compared in order to understand which model has a better estimation of elastic attributes for the coquinas reservoir which has been studied.

- Taking into consideration the availability of 14 sets of dry $V_{p}$ and $V_{s}$ lab measurements of core samples which represent the same facies of the carbonate reservoir profiled by the well logs, the calibrated and validated rock physics models applied using the well log data were also applied using the core samples data in order to estimate the elastic attributes of those samples. However, it is important to highlight that with the core samples, a wider analysis was done. It was possible to validate not only 
$\mathrm{P}$ wave velocities $\left(V_{p}\right)$ but also $\mathrm{S}$ wave velocities $\left(V_{s}\right)$ and $V_{p} / V_{s}$ ratios.

- The rock physics model that had the better performance with respect to the carbonate elastic attributes estimations regarding well logs was chosen to run deterministic sensitivity analyses related to pores aspect ratios, fluids and mineralogical content. The goal of running the sensitivity analyses is to obtain a quantitative sense about how the carbonate elastic attributes change due to the simulations of possible diagenetic processes through the variations in the model input parameters.

Each one of the aforementioned items will be described, explained and discussed in light of all the previous information delivered in Chapters 2, 3, 4 and 5 .

\section{1}

\section{Rock Physics Parameters Evaluation Through the DREAM Algorithm}

Since the prior parameters distributions were already settled and explained, as can be seen in section 5.2, it is possible to input those distributions in the DREAM algorithm and obtain not only the posterior distributions and their properties (MAPs, means and variances) for each parameter but also the information about how correlated those parameters can be. It is important to remember that this approach will be executed only for the well logs. In this way, the Well 49 was chosen to be posed in the Bayesian framework. In fact, in Table 6.1, the means, variances and bounds of each prior parameter distribution are described. All those distributions were explained together with Figures 5.2, 5.3, 5.5, 5.6 and 5.7 in Chapter 5.

Table 6.1: Means, Variances and Lower (LB) and Upper bounds (UB) of the Prior Parameters Distributions

\begin{tabular}{cllcl} 
Parameter & Mean & Variance & LB & UB \\
\hline$V_{1}$ & & & 0 & 0,1 \\
$\alpha_{1}$ & 0,015 & 0,004 & 0,001 & 0,03 \\
$\alpha_{2}$ & 0,4 & 0,1 & 0,01 & 0,8 \\
K (GPa) & 70 & 1,7 & 63,7 & 76,8 \\
G (GPa) & 30 & 0,5 & 28 & 32
\end{tabular}


It is important to mention that the T-Matrix rock physics model was chosen to be illustrated in the next figures because the T-Matrix model demonstrated a better performance over the rock physics model of Xu \& Payne in the DREAM's simulations. In fact, the RMSE of the MAP values of the TMatrix model was lower than the RMSE of the MAP values of the Xu \& Payne approach, as can be seen in Table B.1. In this way, in order to start the analyses of the DREAM's outcomes, Figure 6.1 illustrates the $95 \%$ uncertainty intervals of the T-Matrix rock physics model for Well 49. The black region is related to the parameters uncertainty, whereas the gray region is related to the total uncertainty (parameters uncertainty + model uncertainty). The red dots are the measurements of the $\mathrm{P}$ wave velocities.

The parameters uncertainty (black region) represents the simulations generated by the DREAM algorithm when occur the parameters stabilization around the posterior distributions. For instance, take into consideration that 100 combinations of parameters were found out through the Markov Chains search in the parameters space. Thus, each set $\left(\alpha_{1}, V_{1}, \alpha_{2}, \mathrm{~K}\right.$ and $\left.\mathrm{G}\right)$ of these combinations will generate one simulation, gathering all those 100 simulations regarding those 100 different combinations of parameters, it is possible to generate the black region if we only consider the $95 \%$ interval of confidence. The total uncertainty is the sum of the parameters uncertainty and the model uncertainty. The DREAM algorithm uses the RMSE (Root mean squared error) which is related to the difference between the modeled and the measured $\mathrm{P}$ wave velocities. Therefore, computing the RMSE values for all the 100 aforementioned simulations around the posterior parameters distributions, taking into consideration a normally distributed error and a 95\% interval of confidence, it is possible to generate the gray region.

It is possible to notice in Figure 6.1 that almost all the red dots (measurements) lie in the black or grey regions, inside of the parameters or total uncertainty $95 \%$ intervals of confidence. In fact, only 5 of 232 measurements are out of both regions and the corresponding RMSE (root mean squared error) using the MAP values was approximately $133 \mathrm{~m} / \mathrm{s}$ (Table B.1 in Appendix B). In this way, it is possible to conclude that the T-Matrix rock physics model matched the well log measurements of the $\mathrm{P}$ wave velocities very well for this carbonate reservoir. 


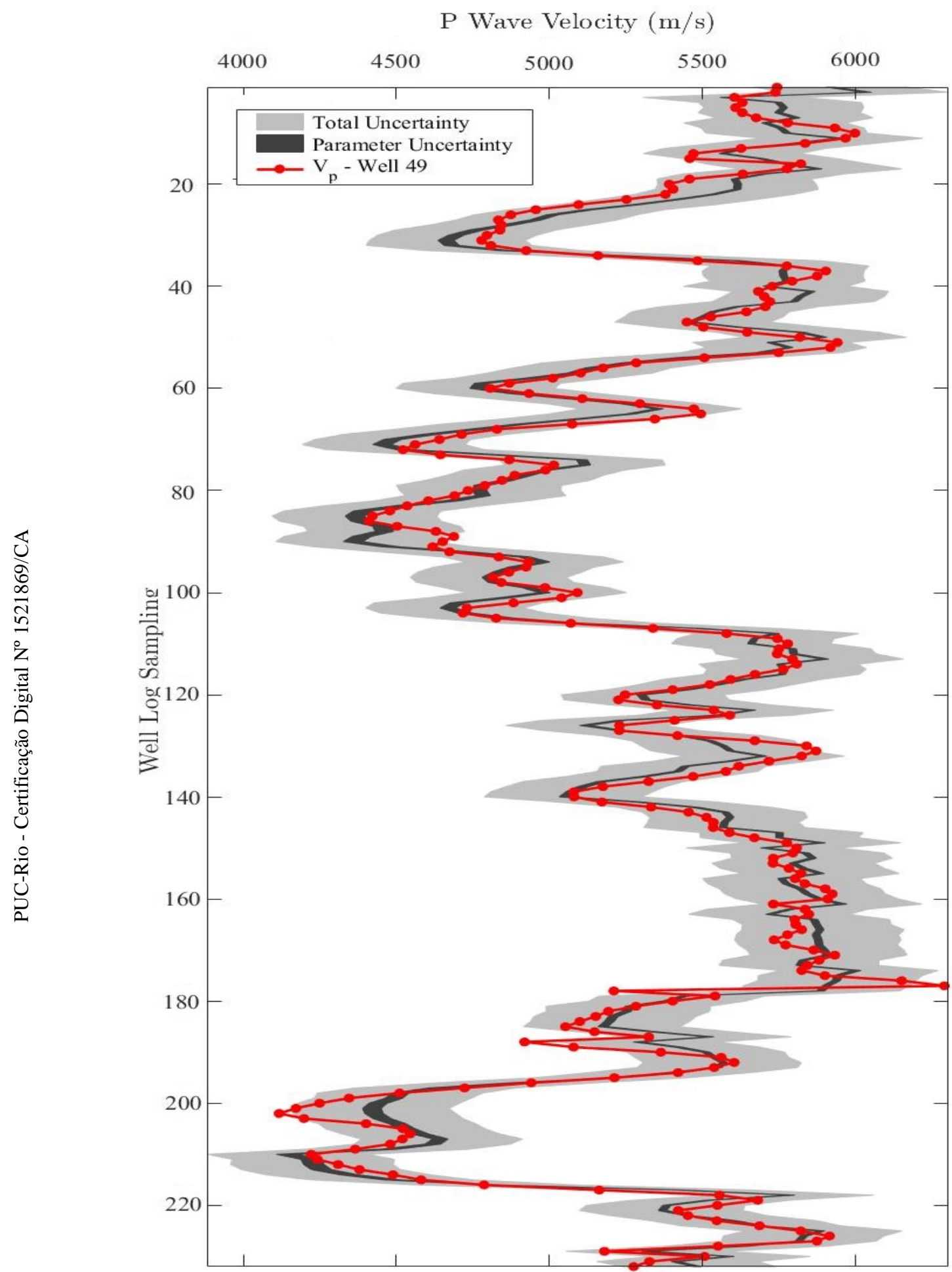

Figure 6.1: Intervals of $95 \%$ uncertainty regarding the parameters (black region) and total uncertainty (gray region) using the T-Matrix Rock Physics Model - Well 49. 
Before continuing the explanation about the DREAM's outcomes, it is important to mention that in Figures 6.2 and 6.3 , the values of $\mathrm{K}$ and $\mathrm{G}$ (coquinas bulk and shear elastic moduli) are in the decimal scale. Those values were settled in this way to facilitate the simulation because it is not intuitive to run the simulations with values in the order of $10^{-1}\left(V_{1}, \alpha_{1}\right.$ and $\left.\alpha_{2}\right)$ and $10^{9}$ ( $\mathrm{K}$ and $\mathrm{G}$ ), because the unit of $\mathrm{K}$ and $\mathrm{G}$ are in GPa (Giga Pascal). Therefore, after the simulation, the decimal values of $\mathrm{K}$ and $\mathrm{G}$ were multiplied for $10^{11}$. In fact, Tables 6.2 and 6.3 already show the values of $\mathrm{K}$ and $\mathrm{G}$ in GPa.

It is also important highlight that Table 6.2 is related to the DREAM's simulations using the T-Matrix rock physics model for the Well 49. On the other hand, in Table 6.3, it is also possible to analyze the DREAM's outcomes for the Well 49 using the Xu \& Payne rock physics model. In this way, taking into consideration the aforementioned information, Figure 6.2 illustrate the histograms of the posteriors parameters distributions, where the blue crosses represent the MAP (maximum à posteriori) values which are described in Table 6.2 with their respective values of variance and standard deviation.
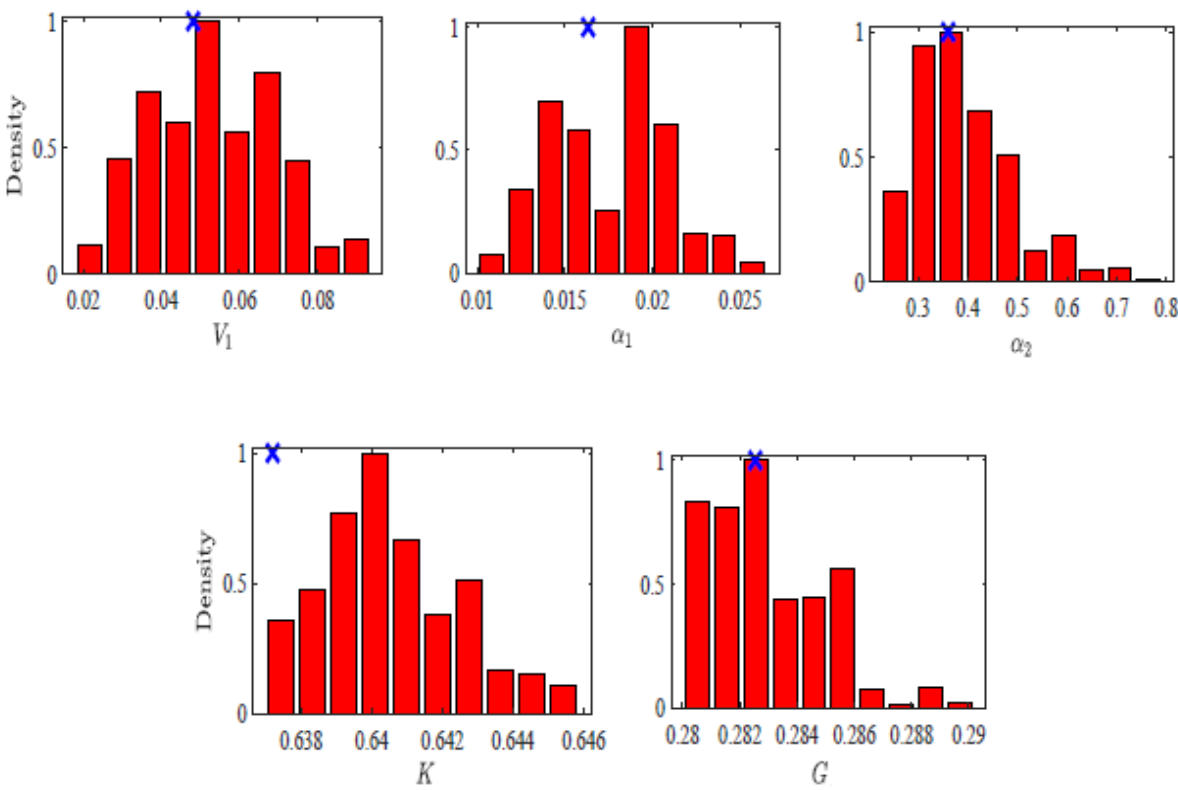

Figure 6.2: Histograms of the marginal posterior distributions of the parameters $V_{1}, \alpha_{1}, \alpha_{2}$, K and G (Well 49) - T-Matrix Model. The blue crosses represent the MAP values. 
Table 6.2: DREAM's Outputs - Well 49 - T-Matrix Model

\begin{tabular}{cccc} 
Parameter & MAP & Mean & Standard Deviation \\
\hline$V_{1}$ & 0,048 & 0,053 & 0,015 \\
$\alpha_{1}$ & 0,016 & 0,017 & 0,003 \\
$\alpha_{2}$ & 0,360 & 0,393 & 0,102 \\
$\mathrm{~K}(\mathrm{GPa})$ & 63,7 & 64,0 & 0,1 \\
$\mathrm{G}(\mathrm{GPa})$ & 28,2 & 28,2 & 0,2
\end{tabular}

Table 6.3: DREAM's Outputs - Well 49 - Xu and Payne Model

\begin{tabular}{cccc} 
Parameter & MAP & Mean & Standard Deviation \\
\hline$V_{1}$ & 0,051 & 0,048 & 0,011 \\
$\alpha_{1}$ & 0,016 & 0,015 & 0,003 \\
$\alpha_{2}$ & 0,404 & 0,408 & 0,099 \\
$\mathrm{~K}(\mathrm{GPa})$ & 63,7 & 63,8 & 0,1 \\
$\mathrm{G}(\mathrm{GPa})$ & 28,3 & 28,3 & 0,2
\end{tabular}

As was already mentioned almost all the inputs in the Bayesian framework through the DREAM algorithm were Gaussian. Despite that, in Figure 6.2 , it is possible to observe three different approximate posterior distributions in the histograms: Gaussian $\left(V_{1}\right)$, Bimodal $\left(\alpha_{1}\right)$ and Log Normal (K, G and $\alpha_{2}$ ). In Figure 6.3, the blue histograms are the marginal posterior distributions of each parameter optimized in the Bayesian framework: $V_{1}, \alpha_{1}, \alpha_{2}, \mathrm{~K}$ and G. In this same figure, it possible to observe through the bivariate scatter plots how the parameters are related to each other. Therefore, it is possible to conclude that there is a strong positive correlation between parameters $V_{1}$ and $\alpha_{1}$ in the simulation using the T-Matrix rock physics model. This correlation may signify that when the values of the cracks aspect ratios $\left(\alpha_{1}\right)$ tend to increase, the volume of those cracks $\left(V_{1}\right)$ also tends to increase. In fact, analyzing Figure 6.3 carefully, it is also possible to realize a smooth correlation between $V_{1}$ and $\alpha_{2}$ which present almost the same behavior already described in the correlation between $V_{1}$ and $\alpha_{1}$. Despite all those aforementioned considerations, analyzing Figure 6.3, all the other parameters do not express any strong positive or negative correlations. As a matter of fact, the other parameters demonstrate approximately zero correlation or slightly positive and/or negative correlations. 


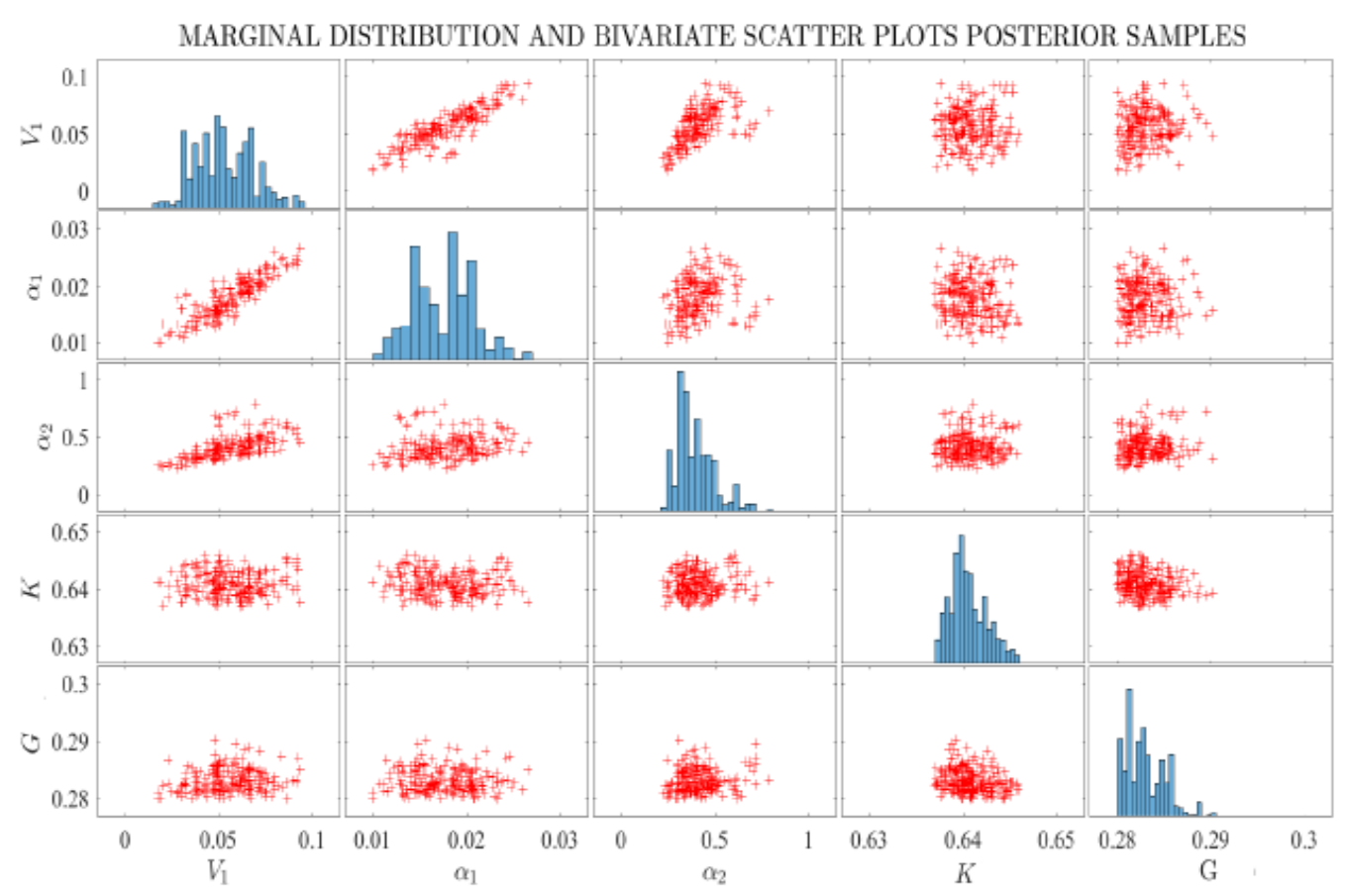

Figure 6.3: Marginal distributions (blue histograms) and bivariate scatter plots regarding the correlation between the parameters of the posterior distributions outputted through the DREAM algorithm (Well 49) - T-Matrix Model.

In order to finish this section, it is important to mention that during the deterministic rock physics modelings using the well logs and core samples measurements in this chapter, both models were inputted with the MAP values provided by the Bayesian framework using each rock physics model. In this way, the MAP values inputted in the T-Matrix and Xu \& Payne regarding well $\operatorname{logs}$ and core samples measurements are shown in Tables 6.2 and 6.3, respectively.

\section{2}

\section{Rock Physics Modelings Validations and Comparisons - Well Logs}

In this section, using the well log data of the Wells 49, 36 and 10, it will be possible to compute and compare the coquinas elastic attributes estimations generated through the rock physics models of $\mathrm{Xu} \&$ Payne and T-Matrix. In fact, using both models, deterministic rock physics modelings will be accomplished. Therefore, after posing the measurements of Well 49 in the Bayesian framework through the DREAM algorithm, it is possible to take the MAP values (values which better represent the posterior parameters 
distributions) and run the elastic attributes estimations not only locally in Well 49 but also extrapolate the models estimations to Wells 10 and 36. The MAP values which were obtained related to Well 49 data through the Bayesian framework are described in Tables 6.2 and 6.3, for the T-Matrix and Xu \& Payne models, respectively, in section 6.1. In this way, in Figure 6.4, the rock physics models of $\mathrm{Xu} \&$ Payne and T-Matrix are settled over the crossplots $V_{p}-\phi$ of Wells 49, 36 and 10, which are colored by water saturation $\log \left(S_{w}\right)$.

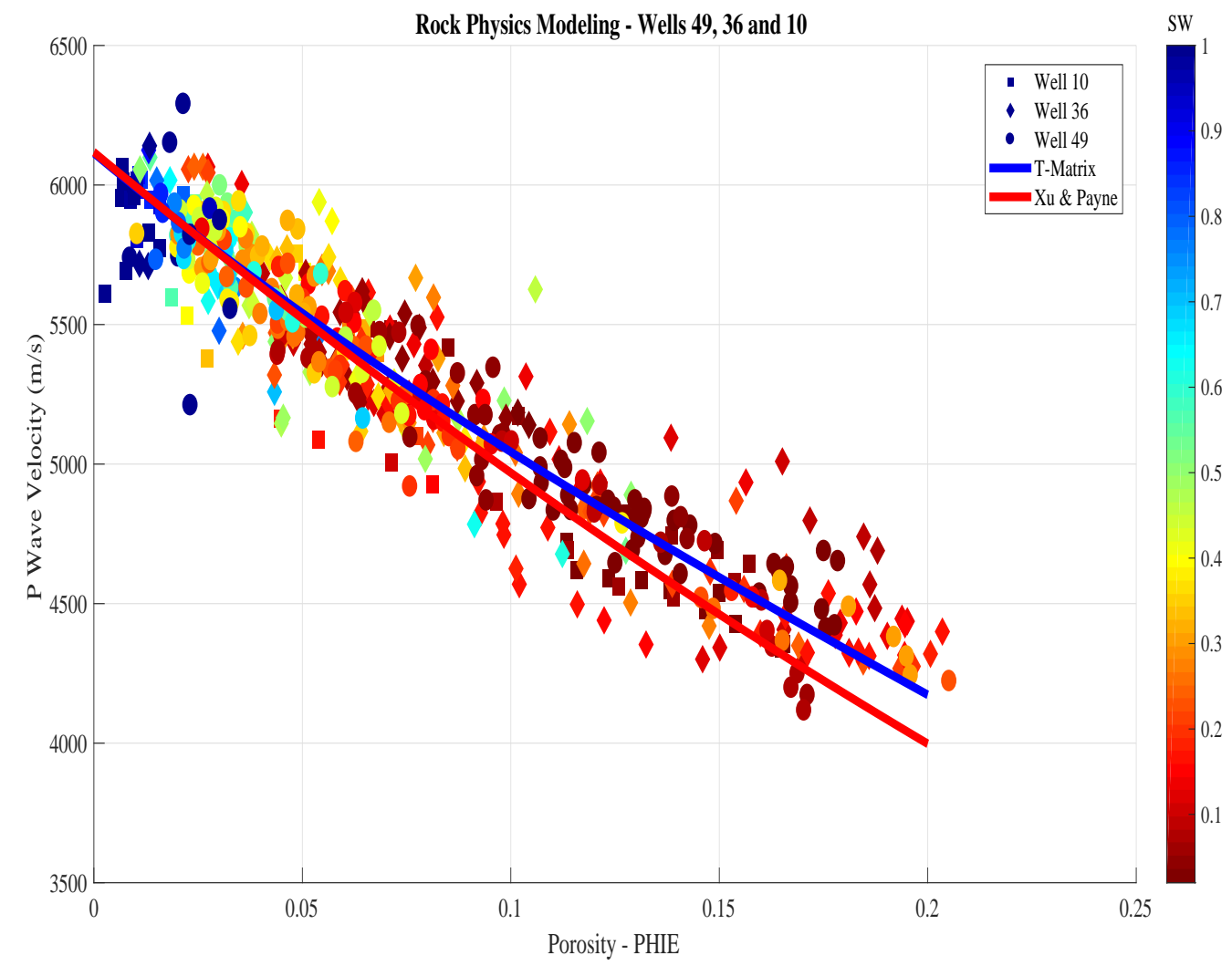

Figure 6.4: Rock Physics Models of Xu and Payne and T-Matrix settled over the crossplots $V_{p}-\phi$ of the Wells 36,49 and 10 colored by the water saturation $\log \left(S_{w}\right)$.

Analyzing Figure 6.4, some important points should be highlighted:

- It is possible to observe that the crossplots $V_{p}-\phi$ of Wells 49,36 and 10 present the same trend.

- The crossplot of Well 49 shows some apparent outliers for low porosities. However, those possible outliers were not removed because they remained in $V_{p}$ measurements even after the cut-offs of $\mathrm{GR}, V_{s h}$ and Caliper. In 
this way, the rock physics modelings and the Bayesian framework were applied taking into consideration those possible outliers.

- In the crossplot $V_{p}-\phi$ of Well 10, it is possible to observe a much more scarcer well log sampling. In fact, the caliper log of this well presented a lot of anomalous high values almost in the entire well log sampling. Thus, those anomalous measurements that may indicate possible washouts were removed from the analysis in order to obtain more accurate elastic attributes estimations.

- As it was expected, both curves have the same start point, and this happens because when the porosity tends to zero, the bulk and shear moduli of the carbonate reservoir tend to be the bulk and shear moduli of the calcite mineral. On the other hand, it is clear that the T-Matrix curve is above of the $\mathrm{Xu} \&$ Payne curve. For higher porosities the Xu \& Payne curve tends to underestimate the $\mathrm{P}$ wave velocities in comparison to the T-Matrix approach where a difference in the order of $200 \mathrm{~m} / \mathrm{s}$ can be observed comparing both models for $\phi=0.2$.

Explain in a concise manner the latter bullet of the aforementioned considerations it will be very important in order to understand the elastic attributes estimations presented in the following pages. In fact, differences in the order of $200 \mathrm{~m} / \mathrm{s}$ can be found out between the rock physics models of $\mathrm{Xu} \&$ Payne and T-Matrix for $\phi=0.2$ in Figure 6.4. Therefore, it was already explained in previous sections, that both models are very different in several aspects. One of these differences which was widely addressed during this dissertation is related to how both models approach fluids inclusions, whereas the T-Matrix uses inclusion based theory, the $\mathrm{Xu} \&$ Payne uses the Gassmann's relations and also inclusion based theory (Section 3.1.3.2). However, the differences of $V_{p}$ for higher porosities of both models are not related to fluids, because the $\mathrm{Xu} \&$ Payne model works in a low frequency perspective due to the Gassmann's relations and the T-Matrix was also settled to estimate elastic attributes in a low frequency perspective too. It is also possible to state that those $V_{p}$ differences with the increase of porosity are not likely to be related to the mineralogical content, because, when the porosity increases the effect of mineralogical content tends to decrease because the pore space occupies the matrix. As a matter of fact, the changes in mineralogical content are likely to be more related to the models intercepts than to their gradients. 
Taking into consideration the information delivered during the Chapter 2 of this dissertation, it is possible to notice that the main factors which alter the elastic attributes in carbonates reservoirs are the: porosity and aspect ratios. The latter, in fact, is one of the reasons why both models for higher porosities are different: The $\mathrm{Xu} \&$ Payne model is grounded in the DEM (Differential effective medium) modeling which is based in ordinary differential equations (ODEs), whereas the T-Matrix is based on the physically integral equation methods (Jakobsen et al., (2003) (53)). Mathematically speaking, differential and integral equations have lot of differences. However, the main point that should be highlighted herein, is that both of them work differently regarding their approximation of the multiple interactions. The T-Matrix rock physics model approximates the multiple interactions by second order correlation, whereas the $\mathrm{Xu} \&$ Payne approach does not work in the same manner (Mavko et al., (2009) (68) and Jakobsen et al., (2003a,b) (54) (53)). Thus, taking into account that the foundations of both models are different and their approximations of the multiple interactions are also different, it is comprehensible a variation between the estimations of both models for higher porosities in Figure 6.4, where more space to store different aspect ratios is provided in the elastic solid media.

After the brief aforementioned highlights and explanations regarding Figure 6.4, it will be possible to better comprehend, analyze and compare the elastic attributes estimations of both rock physics models for Wells 49, 36 and 10 in Figures 6.5, 6.6, 6.7, 6.8, 6.9 and 6.10. Through those figures, it will be shown not only the comparisons between the measured and the modeled $\mathrm{P}$ wave velocities and bulk densities computed by each rock physics model, but also the effective porosity (PHIE) well logs. Besides that, it will be also illustrated the $V_{p}-\phi$ crossplot of each well with the models curves settled over the crossplots and the error histograms (as mentioned in section 4.3) regarding the difference between the modeled and the measured $\mathrm{P}$ wave velocities. It is important to mention that the focus of this section is to analyze the $\mathrm{P}$ wave velocity estimations. Therefore, the statistical analyses regarding the metrics of accuracy and histograms will be limited to the $V_{p}$ estimations, whereas the bulk density estimations and effective porosity logs are illustrated in order to better comprehend and discuss the $\mathrm{P}$ wave estimations of both rock physics models. 

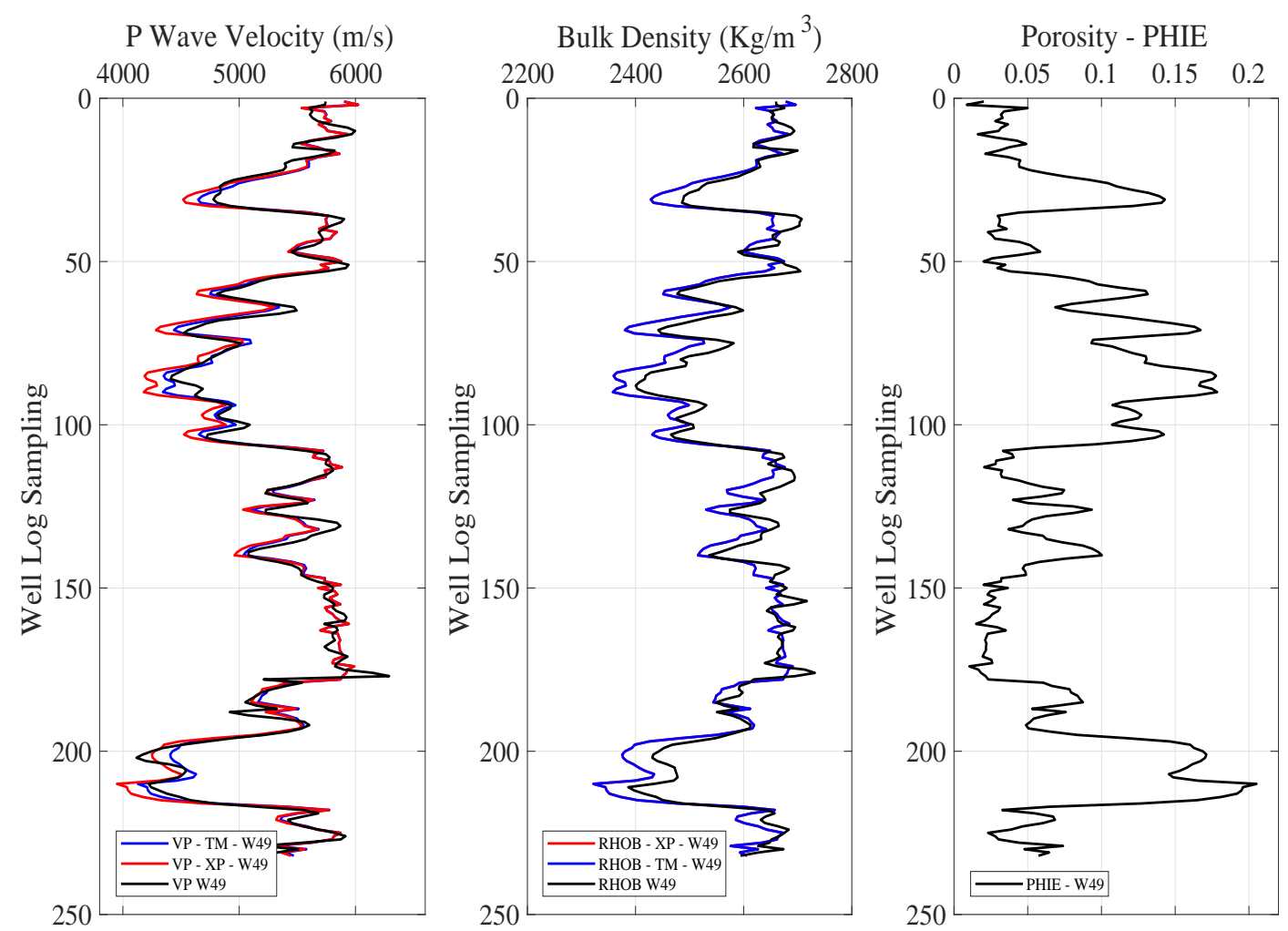

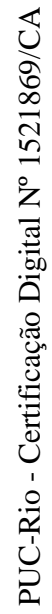

Figure 6.5: Comparison between the elastic attributes estimations of both rock physics models for Well 49 and the effective porosity (PHIE) well log.
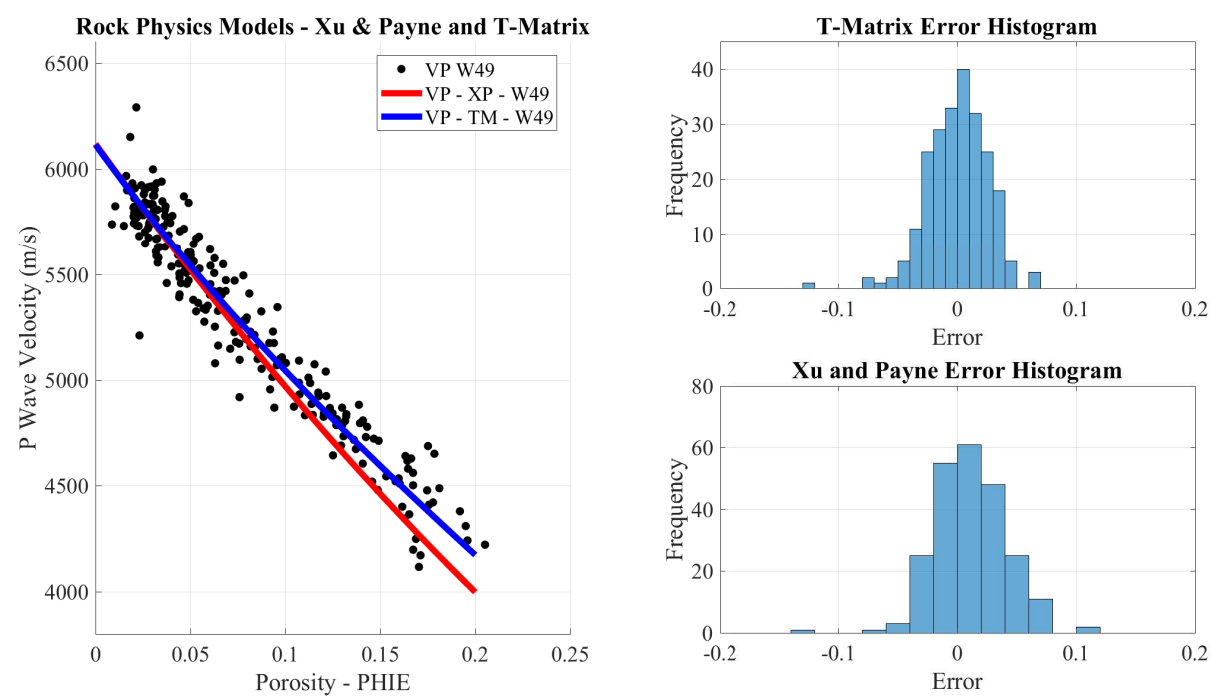

Figure 6.6: Rock physics models curves settled over the crossplot $V_{p}-\phi$ of Well 49 and error histograms regarding the difference between the modeled and the measured $\mathrm{P}$ wave velocities. 

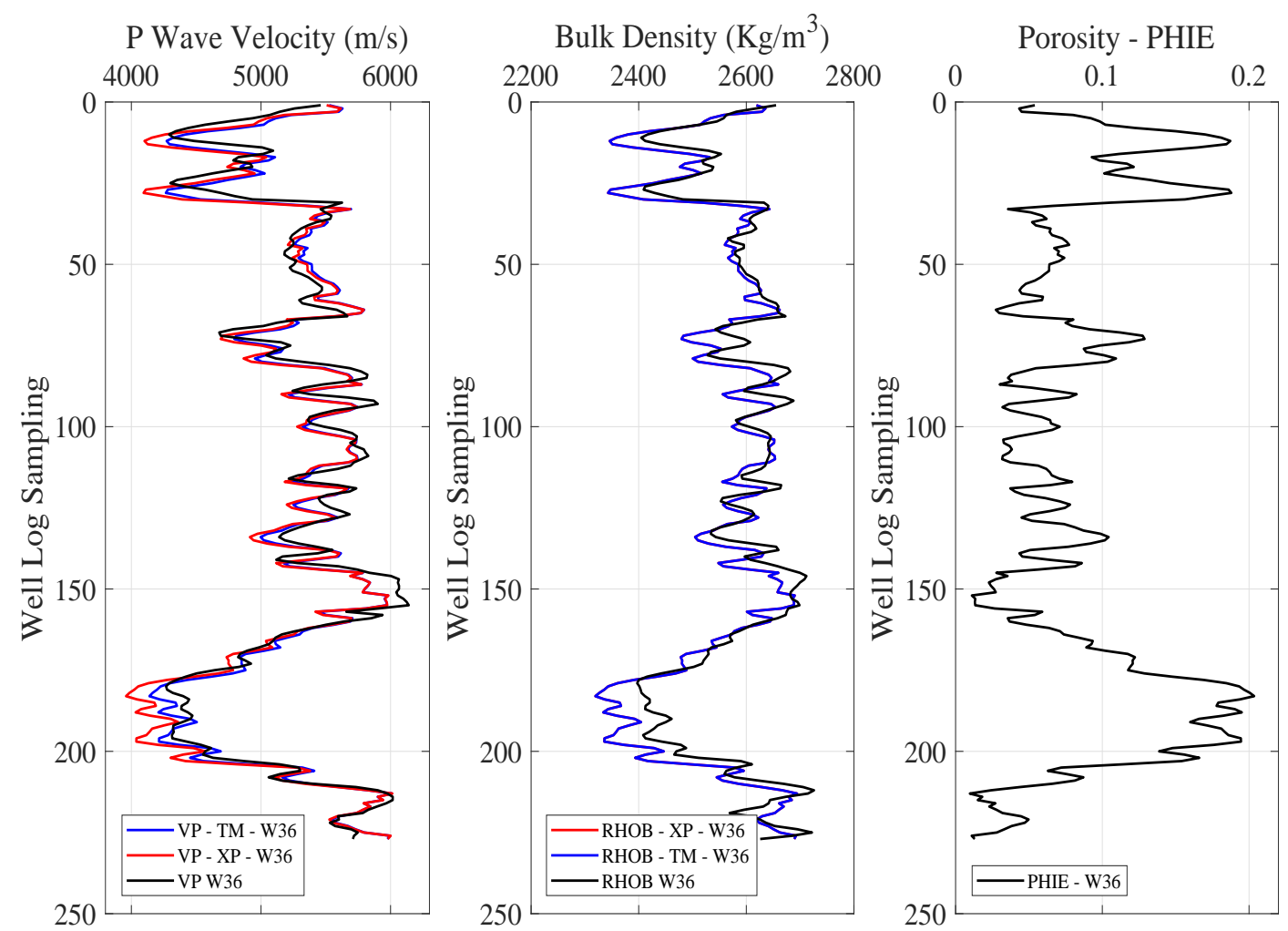

Figure 6.7: Comparison between the elastic attributes estimations of both rock physics models for Well 36 and the effective porosity (PHIE) well log.
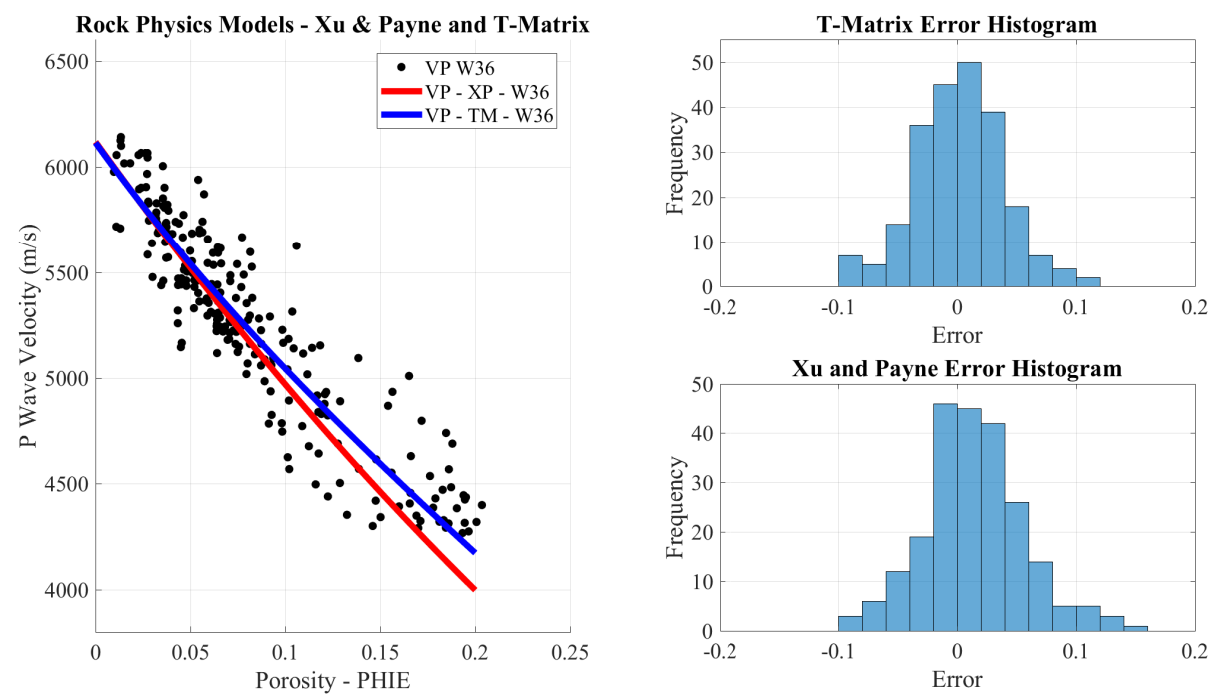

Figure 6.8: Both rock physics models curves settled over the crossplot $V_{p}-\phi$ of Well 36 and error histograms regarding the difference between the modeled and the measured $\mathrm{P}$ wave velocities. 

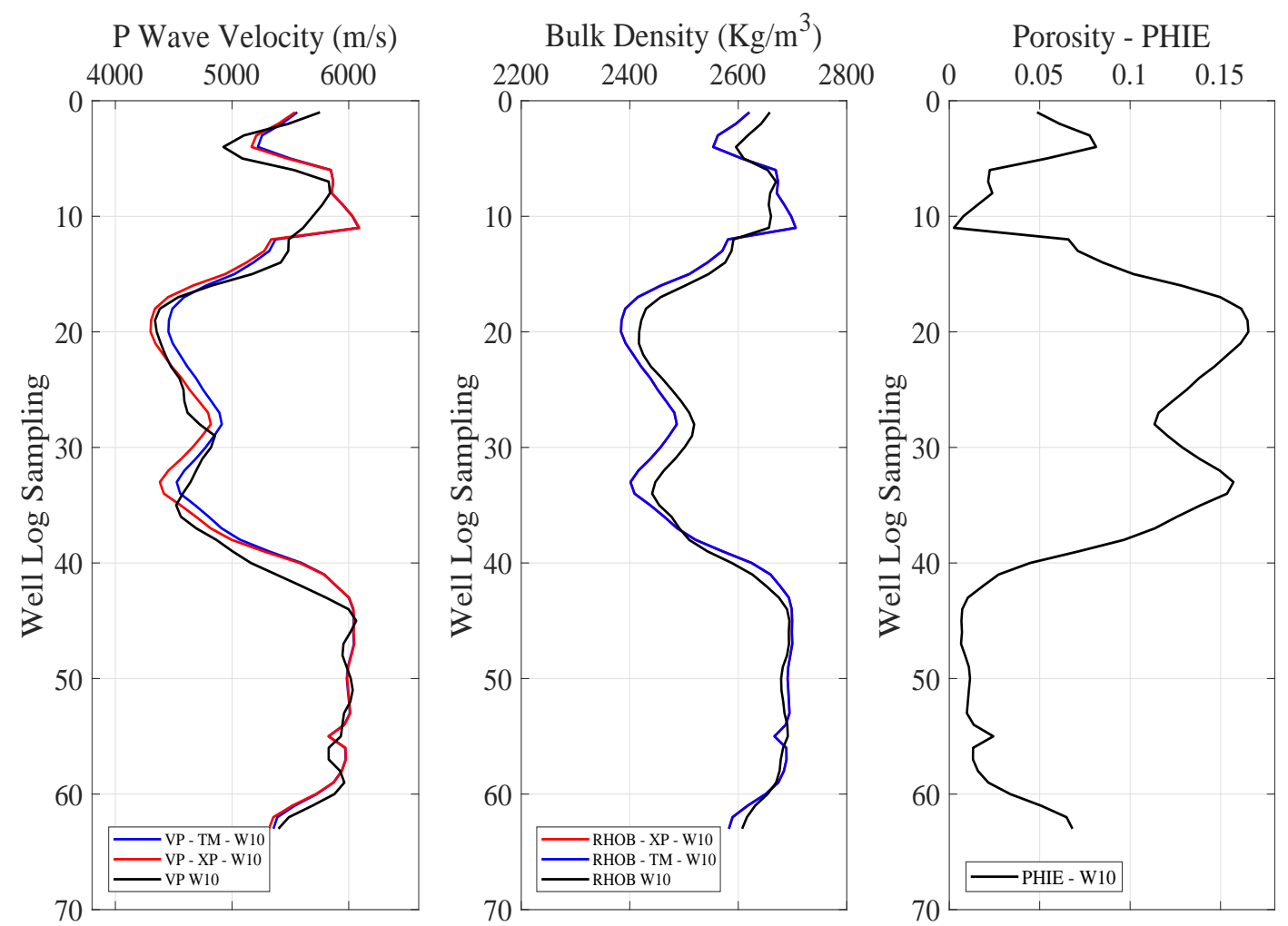

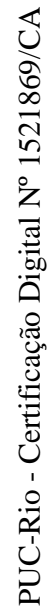

Figure 6.9: Comparison between the elastic attributes estimations of both rock physics models for Well 10 and the effective porosity (PHIE) well log.
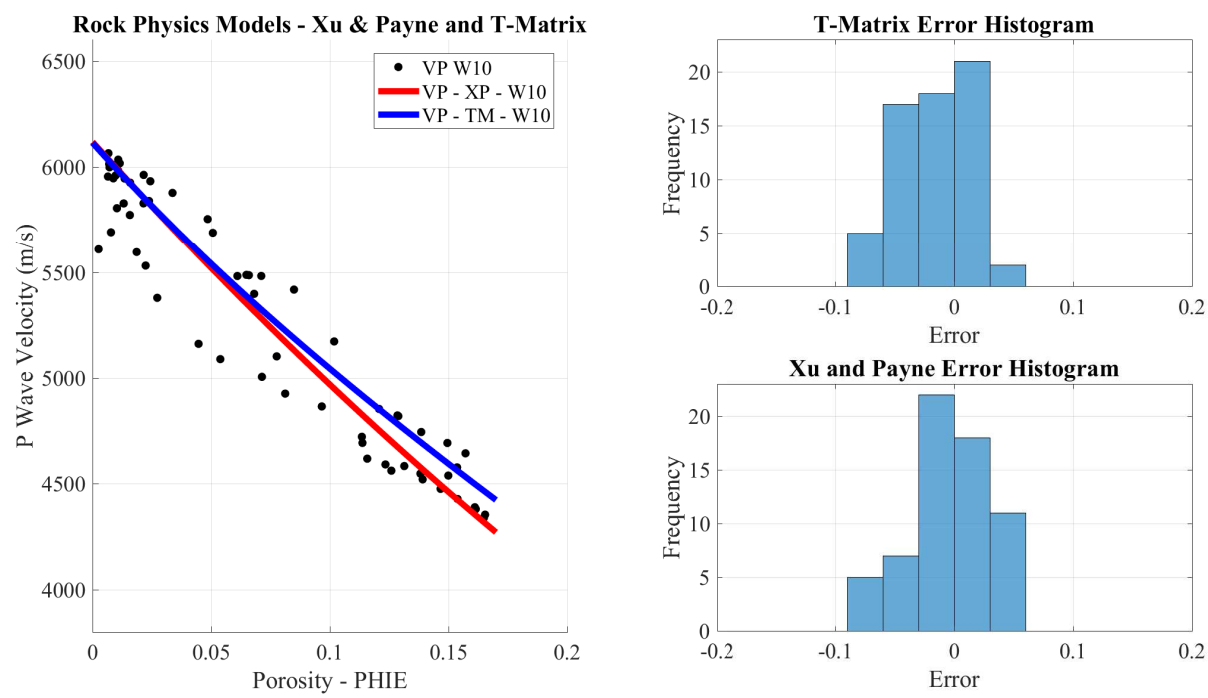

Figure 6.10: Both rock physics models curves settled over the crossplot $V_{p}-\phi$ of Well 10 and error histograms regarding the difference between the modeled and the measured $\mathrm{P}$ wave velocities. 
Therefore, regarding the previous figures, it is very important to make the following considerations:

- The performance of the T-Matrix rock physics model regarding reliable estimations of $V_{p}$ is superior in comparison with the $\mathrm{Xu} \&$ Payne model following the histograms analyzes in Figures 6.6 and 6.8 .

- As it was expected, following the histograms in Figure 6.6, better elastic attributes estimations were obtained in Well 49. This fact is explained because this well log suite was used as calibration data in the Bayesian framework in order to run the parameters evaluation.

- For Wells 49 and 36, the rock physics model of Xu \& Payne generates picks of underestimation for intervals of lower velocities and higher porosities whereas this effect in intervals of higher velocities and lower porosities is much softer (Figures 6.5 and 6.7). That is one of the consequences regarding the underestimations of the $\mathrm{Xu} \&$ Payne model observed in Figure 6.4 for higher porosities.

- In Figures 6.5, 6.7 and 6.9, the bulk densities estimations computed through both rock physics models are superimposed. Besides that, it is also possible to notice that both rock physics models are generating picks of underestimation for intervals of higher porosities whilst for intervals of lower porosities the estimations are more accurate.

The latter bullet of the aforementioned highlights should be explained in a detailed manner. Differently from the $V_{p}$ estimations which are dependent of pores aspect ratios and other intrinsic rock properties, it is possible to estimate the bulk density regardless the information of the rock pore geometry. In fact, both rock physics models estimate bulk densities through the weighted average already described in Chapter 3, Equation 3-43. The bulk density estimation only depends of the matrix, fluid and porosity information. Thus, that is the reason why in Figures 6.5, 6.7 and 6.9 the estimations of the bulk densities of both models are superimposed.

It is also possible to notice, analyzing the same previously mentioned figures, that the bulk densities estimations are underestimated for intervals of higher porosities whereas the estimations for intervals of lower porosities are more accurate. In Chapter 5, specifically in section 5.2.1.1, it was explained 
why a matrix $100 \%$ composed by calcite was taken into consideration in the parameters evaluation through the Bayesian framework and in the rock physics modelings: Most of the XRD analyses showed that the core samples were almost $100 \%$ composed by calcite with lower local presence of the minerals dolomite and quartz. In this way, taking into consideration Table 5.1, the density of the mineral calcite for almost all values of bulk and shear moduli ( $\mathrm{K}$ and $\mathrm{G}$ ) was $2.710 \mathrm{Kg} / \mathrm{m}^{3}$. Thus, this value was used in the rock physics modelings in order to estimate the elastic attributes of the coquinas reservoir rock which has been studied. Taking into account those premises, two possible options explain why both models are underestimating the bulk densities:

1. Despiting the fact that the coquinas reservoir was modeled taking into consideration a matrix $100 \%$ composed by calcite, these results led us to think about a possible mix of minerals in the rock matrix which were not captured in the XRD analyses. Thus, the value of density settled is not representing well the density embedded in the carbonate reservoir.

2. Taking into account that the bad estimations are related to intervals of higher porosities, it is possible to suppose that the $S_{w}$ logs presented some mismeasurements due to the fact that they could had been miscalculated. In this way, impairing the estimations of the bulk densities through both models. In the same way, it is also possible to believe that possible miscalculations of the effective porosity (PHIE) well log can also be impairing the estimations of the bulk densities for both models.

Despite all those considerations about the bulk density, it is important to mention that we could not give up of the choice made of the following very important premise which is corroborated by the XRD measurements: The reservoir rock was composed $100 \%$ of the mineral calcite. It would not be fair to settle the density beyond the values encountered in Table 5.1 only to best fit the model in order to estimate bulk densities. In the sense that we also strict obeyed the values of bulk modulus, shear modulus and density presented in this table in order to provide geological reasoning and boundaries in the optimization process in the Bayesian framework.

After all those explanations regarding the estimations of the bulk densities, in this very moment, it will be possible to verify the performance of both models regarding the $V_{p}$ estimations. In fact, Tables 6.4, 6.5 and 6.6 summarize the metrics of accuracy used to evaluate the $V_{p}$ estimations of 
both rock physics models in Wells 49, 36 and 10. These tables corroborate that the T-Matrix rock physics model has a better performance in the matter of reliable elastic attributes estimations in comparison to the Xu \& Payne model for Wells 49 and 36. In fact, the three metrics of accuracy of Well $49-$ at the well location - show a better performance regarding elastic attributes estimations of the T-Matrix in comparison to the $\mathrm{Xu} \&$ Payne (Table 6.4). The differences are not huge, mainly talking about the mean error, however, in Figure 6.5, it is very clear that the T-Matrix estimations present a more regular behavior than the $\mathrm{Xu} \&$ Payne estimations.

Table 6.4: Metrics of Accuracy - Well 49 - P Wave Velocity

\begin{tabular}{lccc} 
Model & MAE & RMSE & Mean Error \\
\hline T - Matrix & 104,3 & 133,4 & 0,019 \\
Xu Payne & 129,2 & 161,9 & 0,025
\end{tabular}

Table 6.5: Metrics of Accuracy - Well 36 - P Wave Velocity

\begin{tabular}{lccc} 
Model & MAE & RMSE & Mean Error \\
\hline T - Matrix & 152,6 & 193,5 & 0,029 \\
Xu Payne & 172,2 & 220,6 & 0,033
\end{tabular}

Table 6.6: Metrics of Accuracy - Well 10 - P Wave Velocity

\begin{tabular}{lccc} 
Model & MAE & RMSE & Mean Error \\
\hline T - Matrix & 144,6 & 184,1 & 0,028 \\
Xu Payne & 139,6 & 179,9 & 0,027
\end{tabular}

In terms of rock physics extrapolation, through the analyses of the metrics of accuracy of Table 6.5, it is possible to notice that both models have a good power of extrapolation with mean errors around 3\%. Despite the low mean errors for both models, in the estimations of Well 36, in Figure 6.7, the Xu \& Payne model generated two intervals of underestimation, approximately between the well log samplings 0 and 40 and also between the samplings 150 and 220. Certainly, the errors in those intervals are not $3 \%$ and the $\mathrm{Xu} \&$ 
Payne did not generate good estimations at those intervals whereas the TMatrix approach presented much more accurate estimations on those intervals. Well 10, was the only well which the Xu \& Payne had a better performance, however, the metrics for both models are very similar, almost equal. Thus, due to the fact that just a short interval of Well 10 could be analyzed and the metrics are very similar for both models, it is not possible to state that the $\mathrm{Xu}$ \& Payne was really superior. In fact, it can be stated that both models had good elastic attributes estimations for this well.

In a general manner, it is possible to say that the T-Matrix approach demonstrated better elastic attributes estimations in comparison with the $\mathrm{Xu}$ and Payne rock physics model for Wells 49 and 36 due to the picks of underestimation generated by the Xu \& Payne model. However, for Well 10, the estimations of both models were very similar. Therefore, in order to corroborate this statement - T-Matrix is superior over the $\mathrm{Xu} \&$ Payne -, it is very easy to see through histograms analyses that the T-Matrix approach reaches better estimations where most of the errors stand near to $\pm 2 \%$ for Well 49 (Figure 6.6 ), approximately $\pm 4 \%$ for Well 36 (Figure 6.8 ) and around $\pm 2.5 \%$ for Well 10 (Figure 6.10). On the other hand, also through histograms analyses, the $\mathrm{Xu}$ and Payne estimations present most of the errors near to $\pm 4 \%$ in Well 49 (Figure 6.6 ), around $-4 \%$ and $+6 \%$ for Well 36 (Figure 6.8) and approximately $\pm 2.5 \%$ for Well 10 (Figure 6.10 ).

Still regarding the histograms analyses of both rock physics models, it is possible to notice that the difference between the modeled and the measured $V_{p}$ through the rock physics model of $\mathrm{Xu}$ and Payne generates histograms with a wider range of error around $\pm 8 \%$ for Well 49 , approximately $-10 \%$ and $+16 \%$ for Well 36 and around $-9 \%$ and $+6 \%$ for Well 10. On the other hand, the T-Matrix rock physics model presents the following maximum range of errors for Wells 49, 36 and 10, respectively: $-8 \%$ and $+6 \%,-10 \%$ and $+12 \%$, and $-9 \%$ and $+6 \%$ (Figures 6.6, 6.8 and 6.10).

Gathering all the information provided by the histograms and the metrics of accuracy MAE, RMSE and Mean Error, it is possible to state that for this carbonate reservoir rock the T-Matrix approach has a better performance in the matter of elastic attributes estimations than the $\mathrm{Xu} \&$ Payne rock physics model. Furthermore, it was also possible to verify through the analyses of Figures 6.5, 6.7 and 6.9 that the T-Matrix rock physics model had a good parametrization and calibration in Well 49 - at the well location - where most of the errors presented low percentage values and also has a good extrapolation power, hence, well validated for Wells 36 and 10 . 


\section{3}

\section{Rock Physics Modeling Validations and Comparisons - Core Samples}

The approach of estimate the elastic attributes using the rock physics models of T-Matrix and Xu \& Payne for Wells 49, 36 and 10 in the previous section will be also applied in this section using the core samples information. However, it is important to highlight that with the core samples, a wider analysis will be done, not only estimating $\mathrm{P}$ wave velocities $\left(V_{p}\right)$ but also $\mathrm{S}$ wave velocities $\left(V_{s}\right)$ and $V_{p} / V_{s}$ ratios. In this way, in order to achieve such a task, 14 core samples will be addressed in this section and it was possible to obtain dry $V_{p}$ and $V_{s}$ lab measurements for each core sample.

It is important to mention that all those core samples were obtained from different wells, however, all of them have the same carbonate facies of the reservoir profiled by the well logs in Wells 49, 36 and 10: coquinas. Taking into consideration the dry $V_{p}$ and $V_{s}$ measurements from the lab, it was necessary to saturate those measurements through Gassmann's relations already explained in the section 3.1.3.2. The following water saturation value was used in order to saturate the core samples through Gassmann's relations: $S_{w}=0.28$. We reached this value making the averages of the $S_{w} \operatorname{logs}$ of Wells 36 and 49. In fact, Costa et al., (2016) (93) state that Vasquez (2015) (94) showed that lab experiments corroborate the application of Gassmann's relations in coquinas. Grounded in this premise, it was possible to make the saturation through the Gassmann's relations. Besides that, in Figure 6.11, it is possible to observe how the saturated $\mathrm{P}$ wave velocities are settled over the crossplots $V_{p}-\phi$ of the Wells 49, 36 and 10.

Taking into consideration that the core samples are composed by the same carbonate facies of the reservoir which has been studied in this dissertation and the Gassmann's relations work for coquinas, in Figure 6.11, it is possible to notice that the saturated $\mathrm{P}$ wave velocities follow the $V_{p}-\phi$ trend of Wells 49, 36 and 10 very well. In this way, it is possible to plausibly use the MAP values of each parameter $\left(V_{1}, \alpha_{1}, \alpha_{2}, \mathrm{~K}\right.$ and $\left.\mathrm{G}\right)$ optimized in the Bayesian framework using Well 49 data in order to estimate the elastic attributes of the core samples through the rock physics models of Xu \& Payne and T-Matrix. In fact, those MAP values were presented in Tables 6.2 and 6.3 for both models. It is also important to mention an interesting fact regarding Figure 6.11: The saturated $\mathrm{P}$ wave velocities of the carbonate core samples present low values of porosity which vary in the following manner, approximately: $0.02 \leqslant \phi \leqslant 0.09$, whereas the porosities range of Wells 49,36 and 10 presents approximately the following variation: $0.001 \leqslant \phi \leqslant 0.2$. 


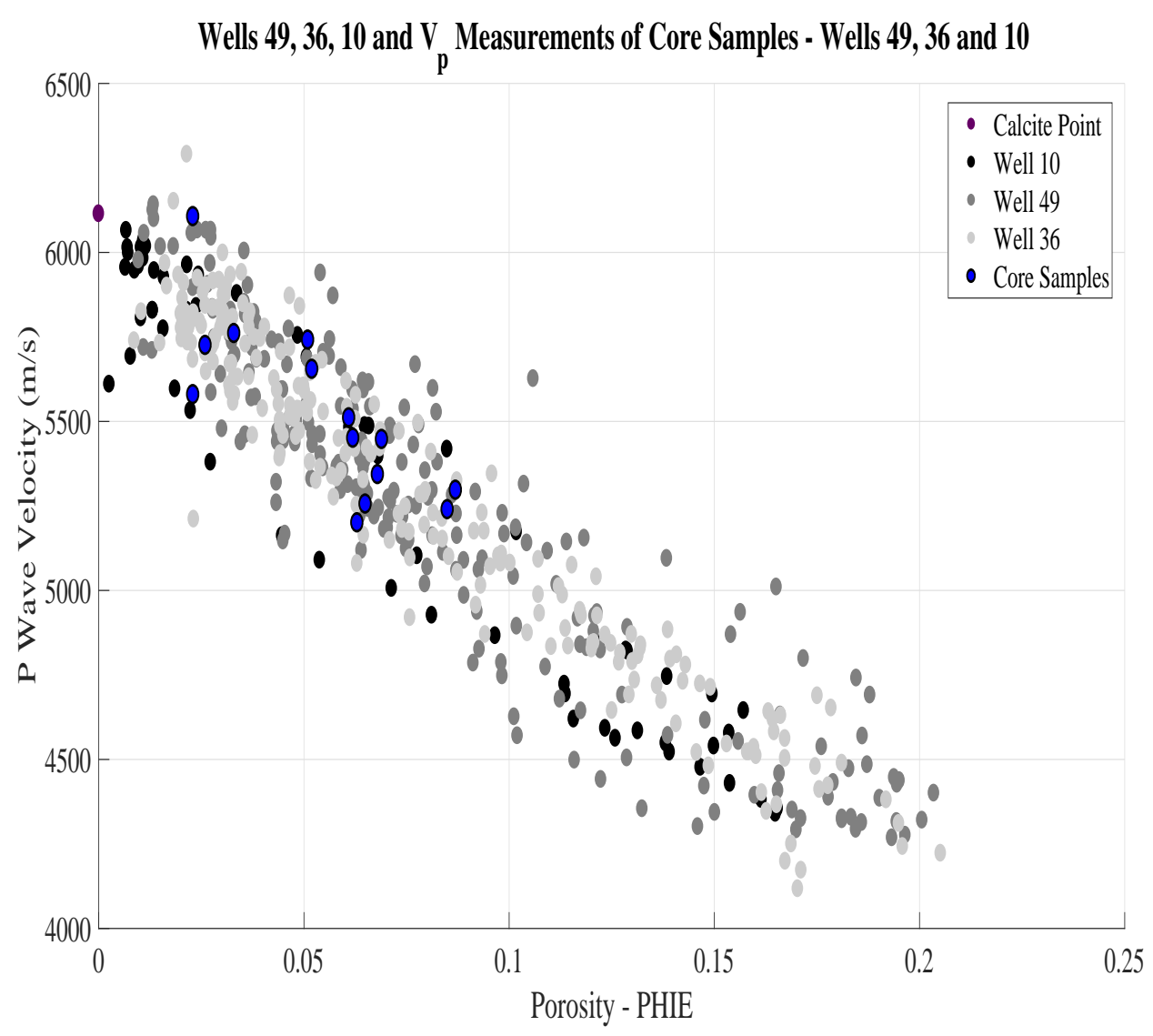

Figure 6.11: Saturated $\mathrm{P}$ wave velocities of the core samples following the trend of the three Wells 49, 36 and 10.

All the aforementioned explanations delivered regarding the core samples will be very important in order to obtain a more clear comprehension of the results in Figures 6.12, 6.13, 6.14, 6.15, 6.16 and 6.17. As a matter of fact, in those figures will be shown the comparisons between the elastic attributes estimations provided by the rock physics models of Xu \& Payne and T-Matrix, their respective error histograms and the models settled over the crossplots $V_{p}-\phi, V_{s}-\phi$ and $V_{p} / V_{s}-\phi$. Besides that, Tables 6.7 and 6.8 summarize the metrics of accuracy (MAE, RMSE and Mean Error) related to each rock physics model for $V_{p}, V_{s}$ and $V_{p} / V_{s}$. Nevertheless, before start the analyses regarding the rock physics modelings using the core samples data, it is also important to explain the meaning of each acronym in the following figures. Therefore, VP|VS|VP/VS CS TM, VP|VS|VP/VS CS XP and VP|VS|VP/VS $\mathrm{CS}$, respectively, signify: the $V_{p}, V_{s}$ and $V_{p} / V_{s}$ estimations obtained through the T-Matrix rock physics model, the $V_{p}, V_{s}$ and $V_{p} / V_{s}$ estimations obtained through the $\mathrm{Xu} \&$ Payne rock physics model and the saturated $V_{p}, V_{s}$ and $V_{p} / V_{s}$ estimations provided by the Gassmann's relations. 

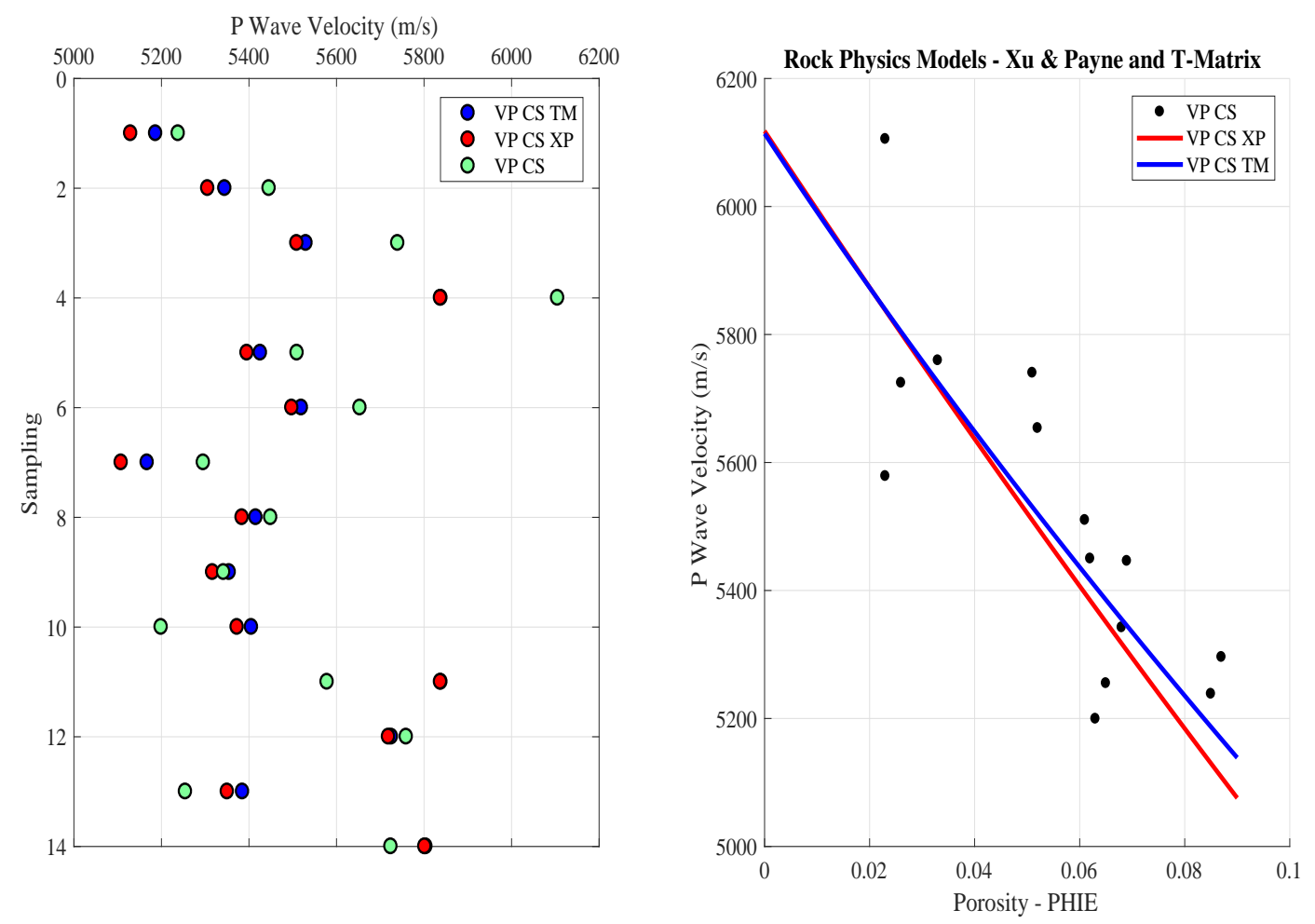

Figure 6.12: Comparison between the elastic attributes estimations of both rock physics models regarding the core samples and the models settled over the crossplot $V_{p}-\phi$.
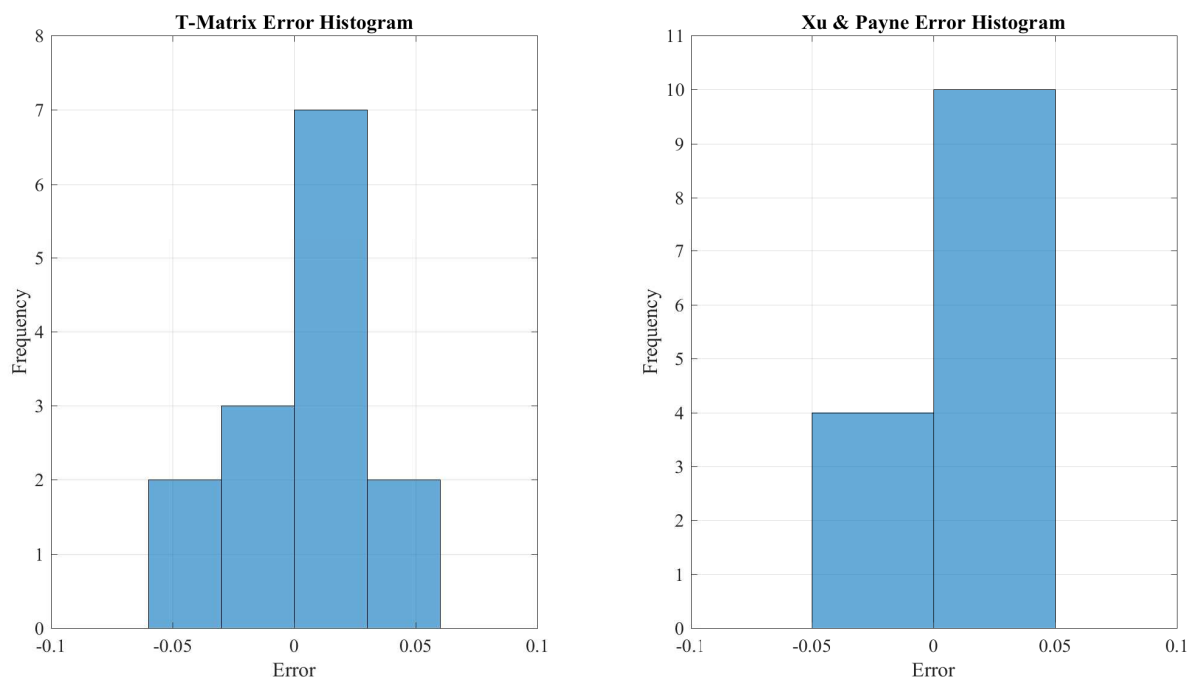

Figure 6.13: Error histograms regarding the difference between the modeled and the measured $\mathrm{P}$ wave velocities for both models. 

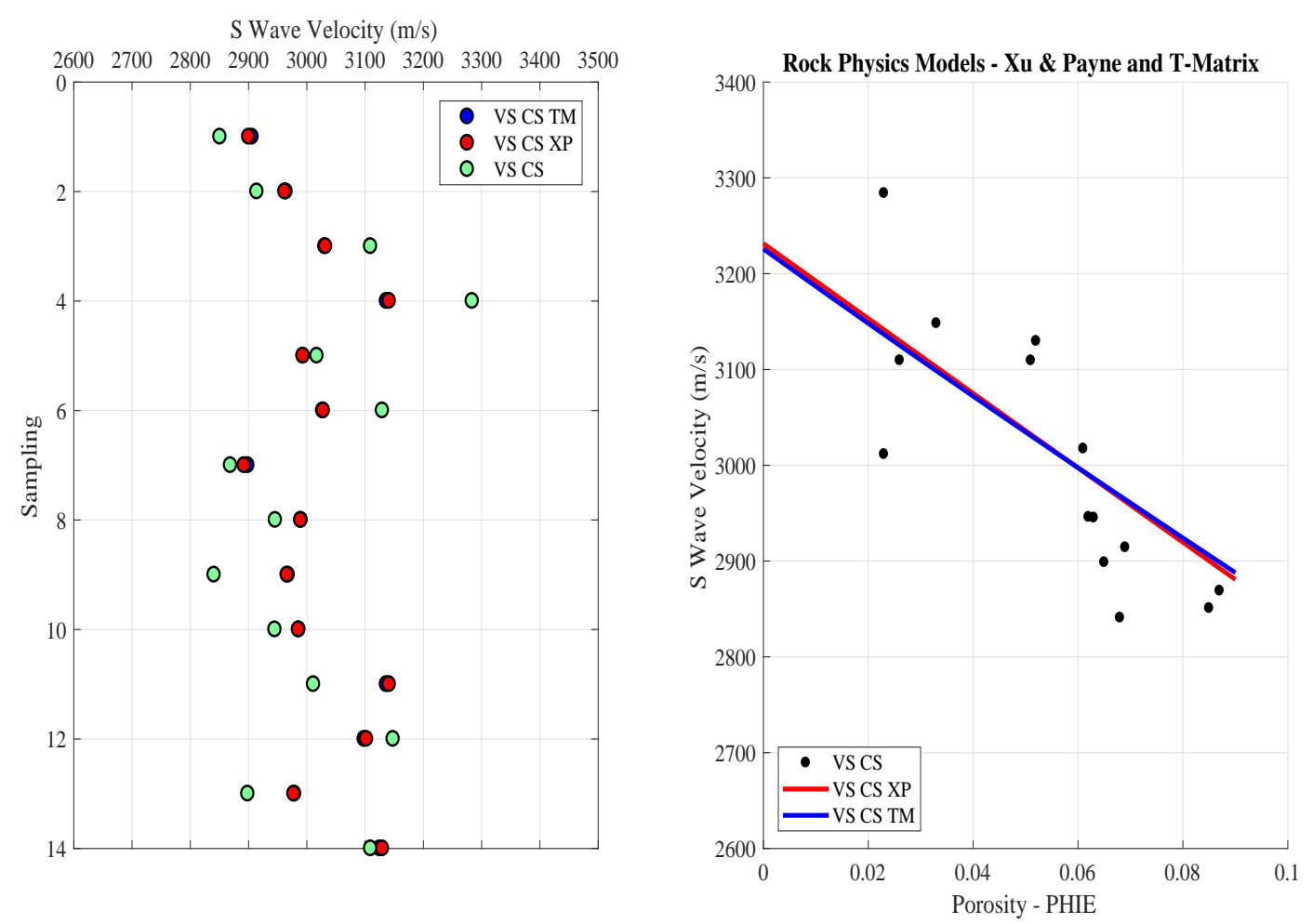

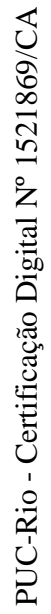

Figure 6.14: Comparison between the elastic attributes estimations of both rock physics models regarding the core samples and the models settled over the crossplot $V_{s}-\phi$.
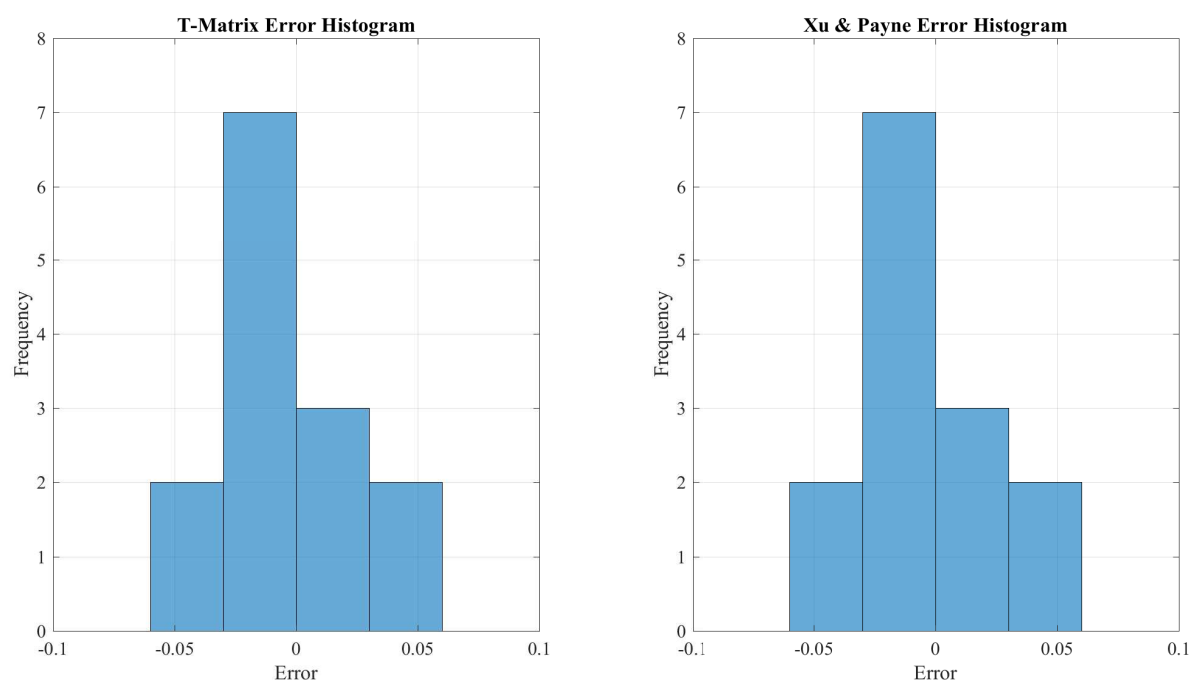

Figure 6.15: Error histograms regarding the difference between the modeled and the measured $\mathrm{S}$ wave velocities for both models. 

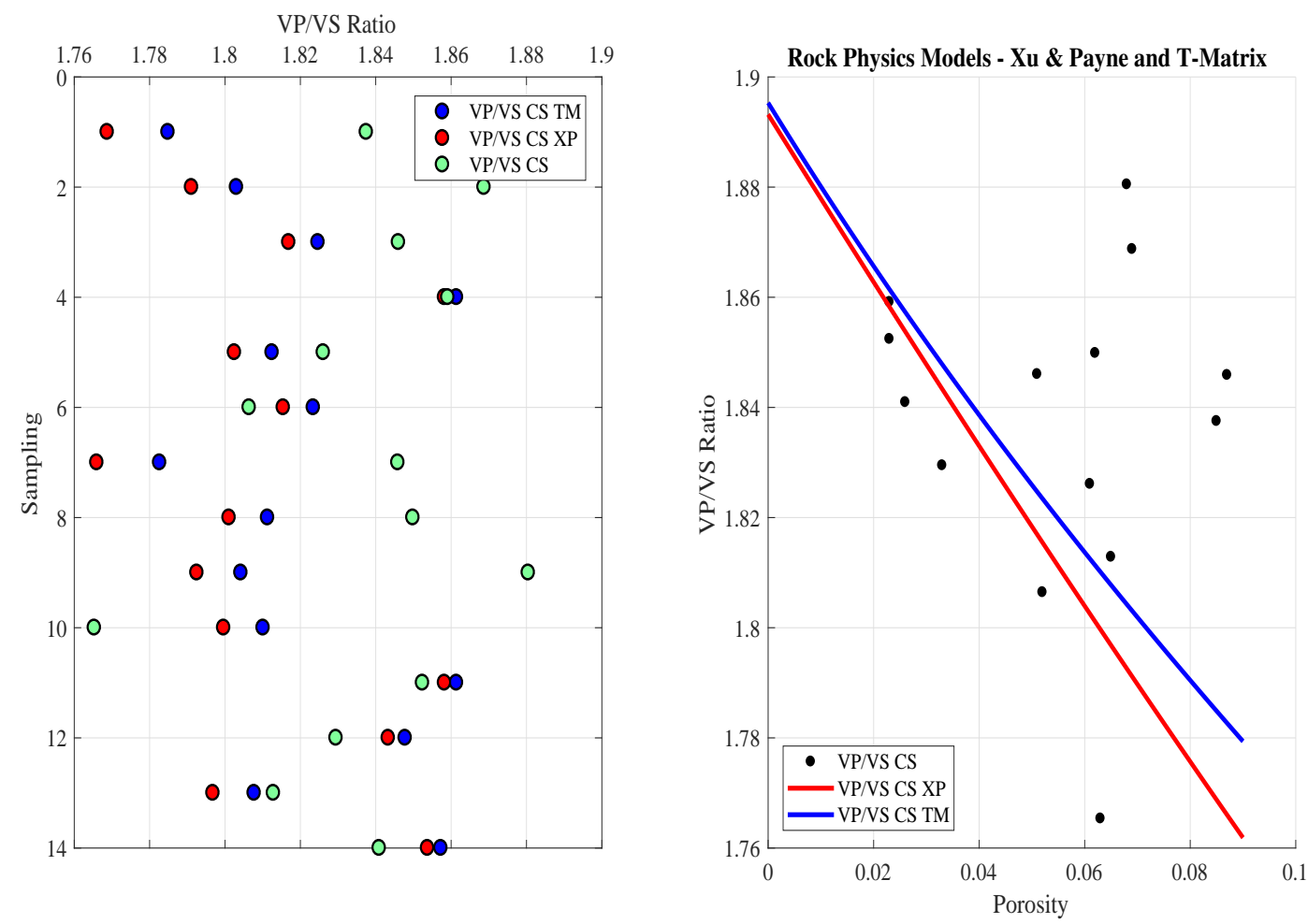

Figure 6.16: Comparison between the elastic attributes estimations of both rock physics models regarding the core samples and the models settled over the crossplot $V_{p} / V_{s}-\phi$.
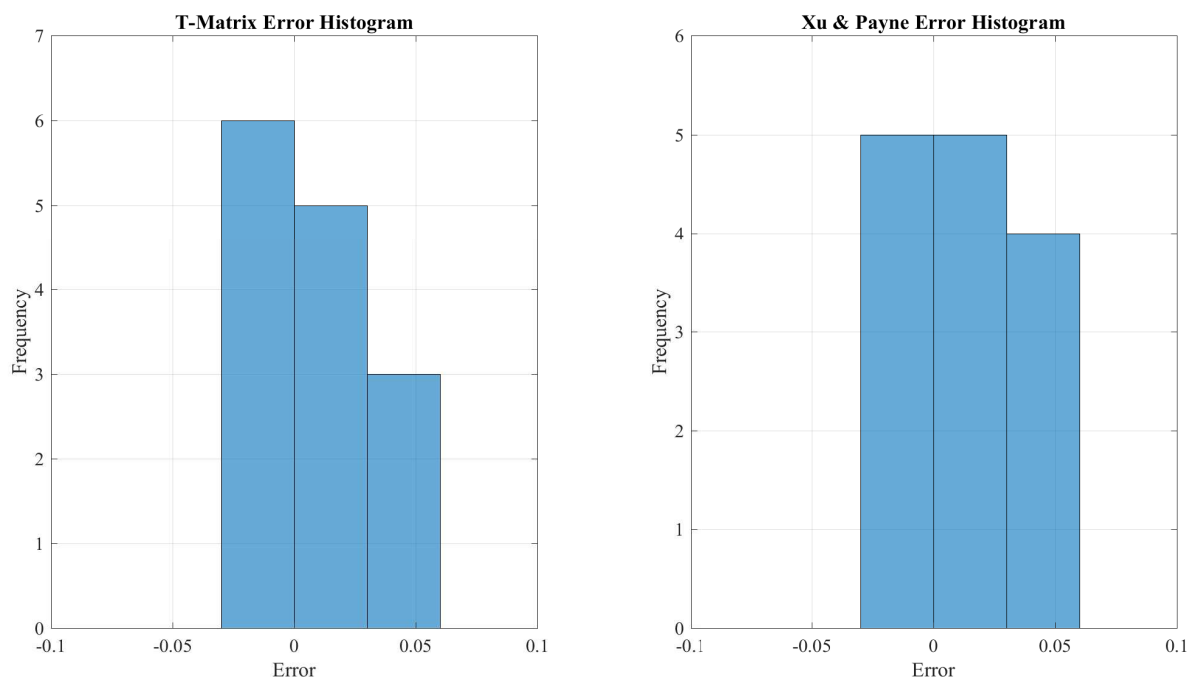

Figure 6.17: Error histograms regarding the difference between the modeled and the measured $V_{p} / V_{s}$ ratio for both models. 
Table 6.7: Metrics of Accuracy - Core Samples - Xu \& Payne

\begin{tabular}{llcc} 
Xu Payne & MAE & RMSE & Mean Error \\
\hline VP & 138,7 & 157,6 & 0,025 \\
VS & 67,8 & 79,94 & 0,022 \\
$V_{p} / V_{s}$ & 0,036 & 0,046 & 0,020
\end{tabular}

Table 6.8: Metrics of Accuracy - Core Samples - T-Matrix

\begin{tabular}{llcc} 
T-Matrix & MAE & RMSE & Mean Error \\
\hline VP & 123,7 & 147,7 & 0,022 \\
VS & 69,3 & 80,17 & 0,023 \\
$V_{p} / V_{s}$ & 0,031 & 0,039 & 0,017
\end{tabular}

Analyzing and relating the results in Figures 6.12, 6.13, 6.14, 6.15, 6.16 and 6.17 to Tables 6.7 and 6.8 , some important insights are obtained:

- Analyzing the histograms, most of the errors regarding $V_{s}$ and $V_{p} / V_{s}$ ratio estimations were in the order of $\pm 2.5 \%$ (Figures 6.15 and 6.17 ) for both models. Only in the $V_{p}$ estimations, most of the errors were around $\pm 2.5 \%$ using the T-Matrix approach, whereas the $\mathrm{Xu} \&$ Payne model presented most of the errors in the order of $\pm 5 \%$ (Figure 6.13 ).

- In Tables 6.7 and 6.8, mean errors found out for $V_{p}$ and $V_{s}$ were around $2.2 \%$ and for $V_{p} / V_{s}$ was approximately $2 \%$. Regarding the metrics of accuracy MAE and RMSE in Tables 6.7 and 6.8, the T-Matrix model demonstrated better elastic attributes estimations for $V_{p}$ and $V_{p} / V_{s}$ whereas for $V_{s}$ both models had similar performances regarding reliable elastic attributes estimations where the Xu \& Payne approach showed to be slightly better in comparison with the T-Matrix.

- Despite the fact that the inputs used in order to estimate the elastic attributes of the core samples were obtained in the Bayesian framework using the data of Well 49, good elastic attributes estimations were obtained as it was possible to see in the previous two bullets regarding the metrics of accuracy and the histograms analyses. 


\section{4 \\ Sensitivity Analyses}

This section is very important for the whole scope of this dissertation. Despite the fact that both models were used to compute the $\mathrm{P}$ and $\mathrm{S}$ wave velocities of the coquinas reservoir using well logs and core samples, in sections 6.2 and 6.3 , in a general manner, it was possible to verify that the T-Matrix rock physics model had a better performance regarding elastic attributes estimations. Therefore, the T-Matrix model was also used in this section to run the sensitivity analyses regarding different pores aspect ratios, fluids and mineralogical content. The results obtained in sections 6.2 and 6.3 demonstrated that the T-Matrix rock physics model can estimate with a low error, the carbonate elastic attributes not only locally in Well 49, but also using the data of Wells 36, 10 and core samples, showing a good power of extrapolation. In this way, it is possible to state that this calibrated, parametrized and validated rock physics model can represent the elastic attributes of this carbonate reservoir facies very well or at least with a good level of accuracy.

Taking into consideration the calibration of the rock physics model at the well location (Well 49) and accurate elastic attributes estimations in other wells through the rock physics extrapolation, it is possible to state that any changes in the input parameters of the calibrated T-Matrix rock physics model would generate the same outcome that the real reservoir would generate if were possible to change the intrinsic characteristics of the reservoir in the real environment. In this way, the motivation to run the sensitivity analyses is to try to simulate different scenarios that may happen away from the well location. Regarding heterogeneous carbonate reservoirs, it is very important to understand what may happen - in a quantitative manner - if the fluids change or if some diagenetic process of dissolution or even silicification/dolomitization also occurs.

Several scenarios can be simulated through the T-Matrix changing the input parameters, for instance: increasing the pores aspect ratio $(\alpha)$, it is possible to simulate a kind of dissolution process because this diagenetic process tends to generate moldic pores in the carbonate rocks, changing the fluids bulk modulus $\left(K_{f l}\right)$ and density $\left(\rho_{f l}\right)$, it is possible to simulate different kind of fluids and altering the bulk and shear moduli ( $K$ and $G$ ) of the rock matrix, it is possible to simulate diagenentic processes of dolomitization and or silicification. Therefore, each one of those will be approached in sections 6.4.1, 6.4.2 and 6.4.3, respectively. Nevertheless, it is important to mention that the start point of the three sensitivity analyses will be the validated T- 
Matrix rock physics modeling in Well 36 illustrated in Figure 6.7, where the input parameters are illustrated in Table 6.2.

\subsection{1}

\section{Sensitivity Analyses - Pores Aspect Ratios}

In sections 6.2 and 6.3 , it was possible to analyze the elastic attributes estimations of both rock physics models for Wells 49, 36 and 10 and for the core samples. In this way, during this section, sensitivity analyses regarding different aspect ratios will be conducted. In fact, only one well and one rock physics model will be approached: The T-Matrix rock physics model in Well 36. Through this model it was possible to obtain the best elastic attributes estimations in comparison to the other results provided by the $\mathrm{Xu} \&$ Payne approach as was already explained in the introduction of this chapter. Taking into consideration that the parameters inputted in this model are presented in Table 6.2, during the sensitivity analyses conducted in this section, only the aspect ratio $\left(\alpha_{1}\right)$ will be changed and the other parameters will be kept constant. It is important to remind that this aspect ratio $\left(\alpha_{1}\right)$ represents approximately $5 \%$ of carbonate pore space volume. Therefore, Figures 6.18 and 6.19 illustrate the first sensitivity analysis regarding different aspect ratios:
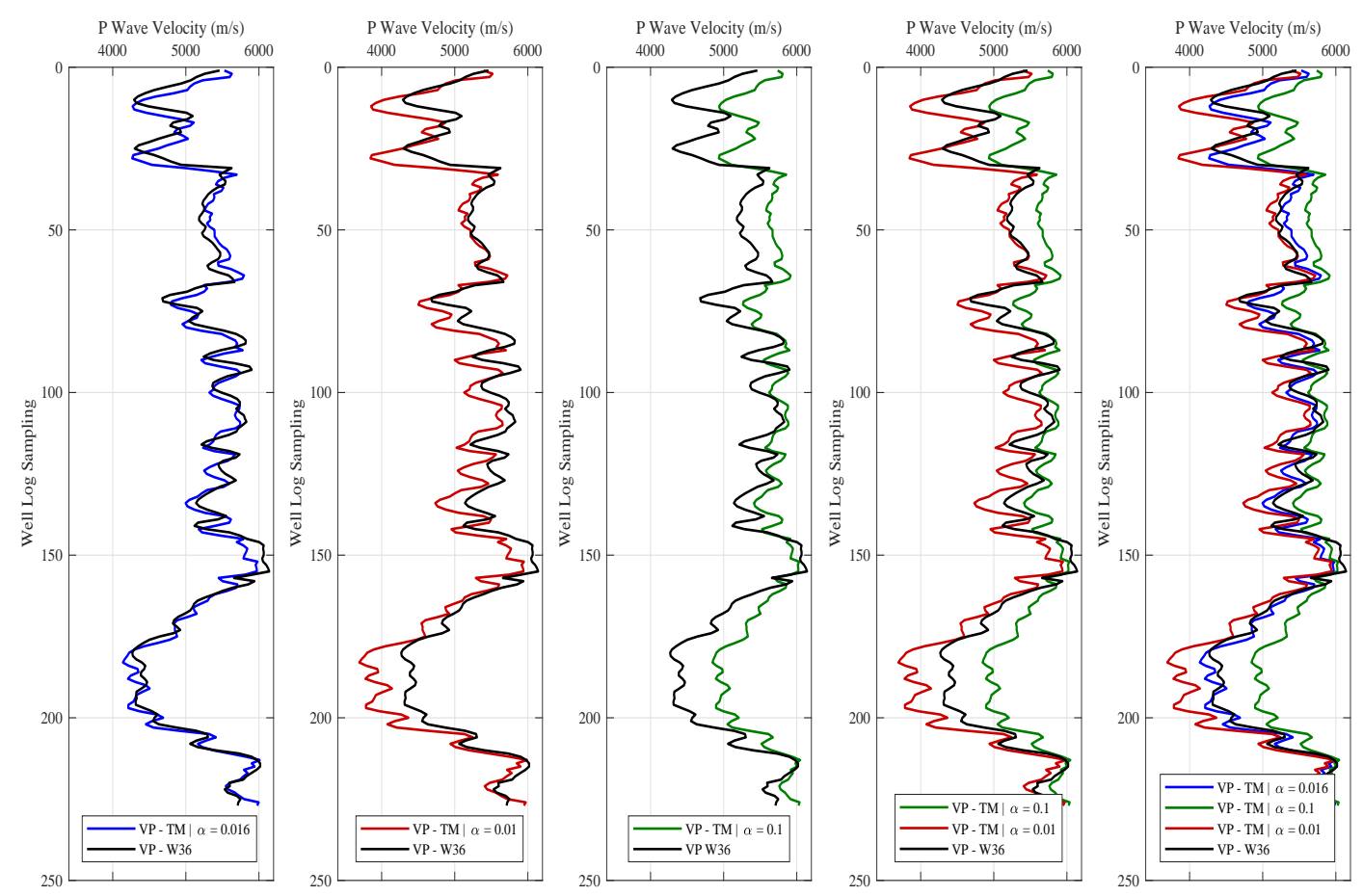

Figure 6.18: Sensitivity analyses regarding different aspect ratios in the rock physics model of T-Matrix with $\alpha=0.016,0.01$ and 0.1 . 


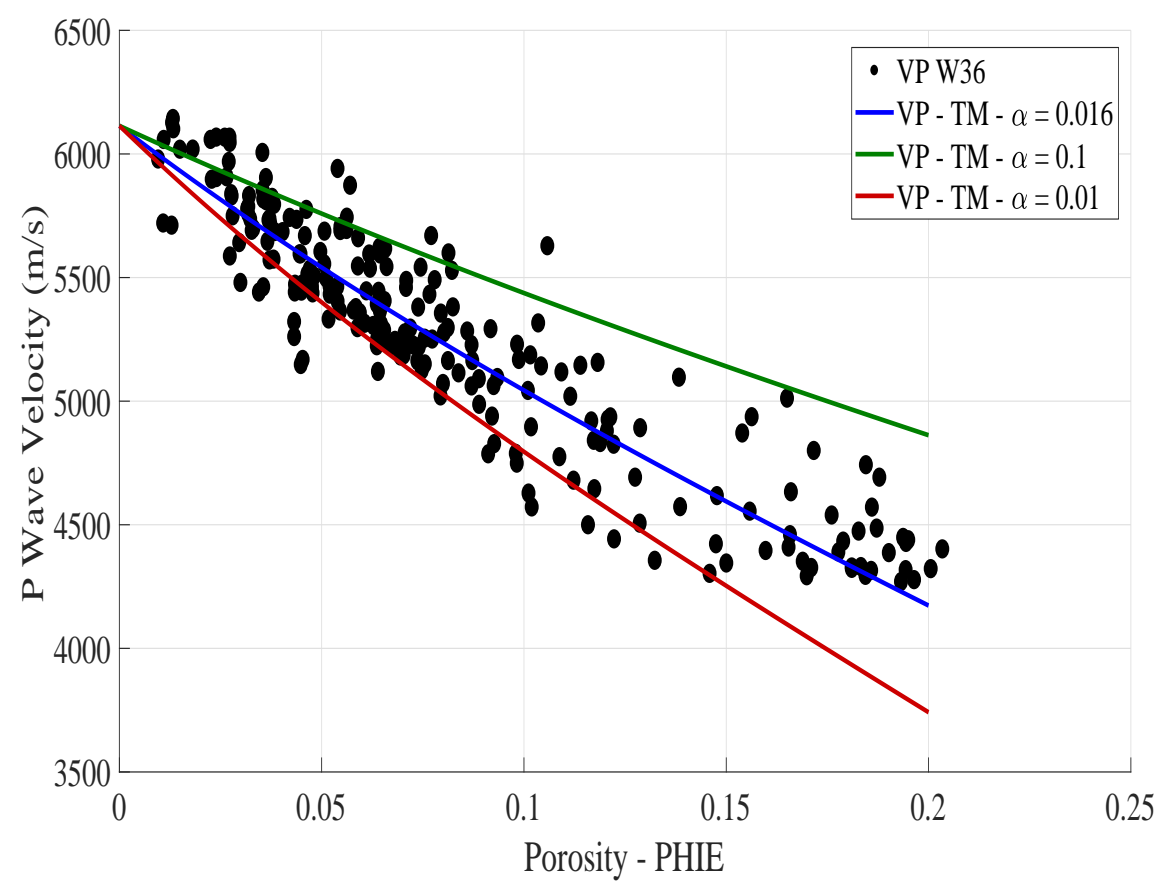

Figure 6.19: Rock physics modelings regarding different aspect ratios using the rock physics model of T-Matrix with $\alpha=0.016,0.01$ and 0.1 over the crossplot $V_{p}-\phi$ of Well 36 .

Figure 6.18 is illustrating what happens when approximately $5 \%$ of the total volume of the carbonate reservoir rock aspect ratio is changed. Therefore, throughout the first three tracks from left to right it is possible to see the comparison between the real $V_{p}$ well log (black) with the rock physics modelings with $5 \%$ of the coquinas pore volume with $\alpha=0.016,0.01,0.1$. In this way, the first track from the left to right represents the rock physics modeling estimation of Figure 6.7 (The validated T-Matrix) with the real $V_{p}$ well log. Comparing the first and second tracks, it is possible to see the huge difference between the elastic attributes estimations for $\alpha=0.016$ and $\alpha=0.01$, as the reservoir gets much softer with this kind of pore type only occupying $5 \%$ of total rock volume. In the same way, when $\alpha=0.1$, the rock gets much stiffer and it is possible to see differences between the first and the third tracks around $500 \mathrm{~m} / \mathrm{s}$. Nonetheless, in Figure 6.19, it is also possible to observe how the T-Matrix rock physics modelings using different aspect ratios are settled over the crossplot $V_{p}-\phi$ of Well 36 .

It is possible to state that the variation of $5 \%$ of the aspect ratios pore volume from 0.016 to 0.01 and 0.016 to 0.1 are enough to generate huge changes in the rock physics model estimations. In fact, the first track in Figure 6.18, the measured $V_{p}$ and the modeled $V_{p}$ are almost superimposed throughout the well $\log$. The only interval with a bad estimation is related to the log first samples. 
Despite all this, comparing the second and third tracks to the first one, it is very easy to find differences of velocities in the order of $600 \mathrm{~m} / \mathrm{s}$. Finally, the last two tracks in Figure 6.18 show an illustrative comparison between all the modelings generated.

In Figure 5.4, in Chapter 5, among the 105 pores aspect ratios measured in the 22 thin section images, it is possible to notice that the predominant volume is represented by the aspect ratios included in the following range: $0.1 \leqslant \alpha \leqslant 0.8$. Taking into consideration that crack pores make the carbonate rock softer and moldic pores make the same rock stiffer, it was possible to observe in Figure 6.18 that the slight change of $\alpha=0.016$ to $\alpha=0.01$ hugely affects the carbonate elastic attributes mainly for low velocities intervals where the porosities tend to be higher. In this way, it should be interesting to run a further analysis addressing the effects of moldic or stiff pores in the carbonate elastic attributes through the T-Matrix rock physics model. Therefore, it will be possible to analyze in quantitative manner how the carbonate elastic attributes change due to the presence of stiff pores. Thus, in Figures 6.20 and 6.21, it is possible to see how the $\mathrm{P}$ wave velocity of the carbonate reservoir changes when approximately $5 \%$ of the reservoir pore volume has the following values of aspect ratio: $\alpha=0.016,0.1,0.2,0.4,0.6,0.8$.
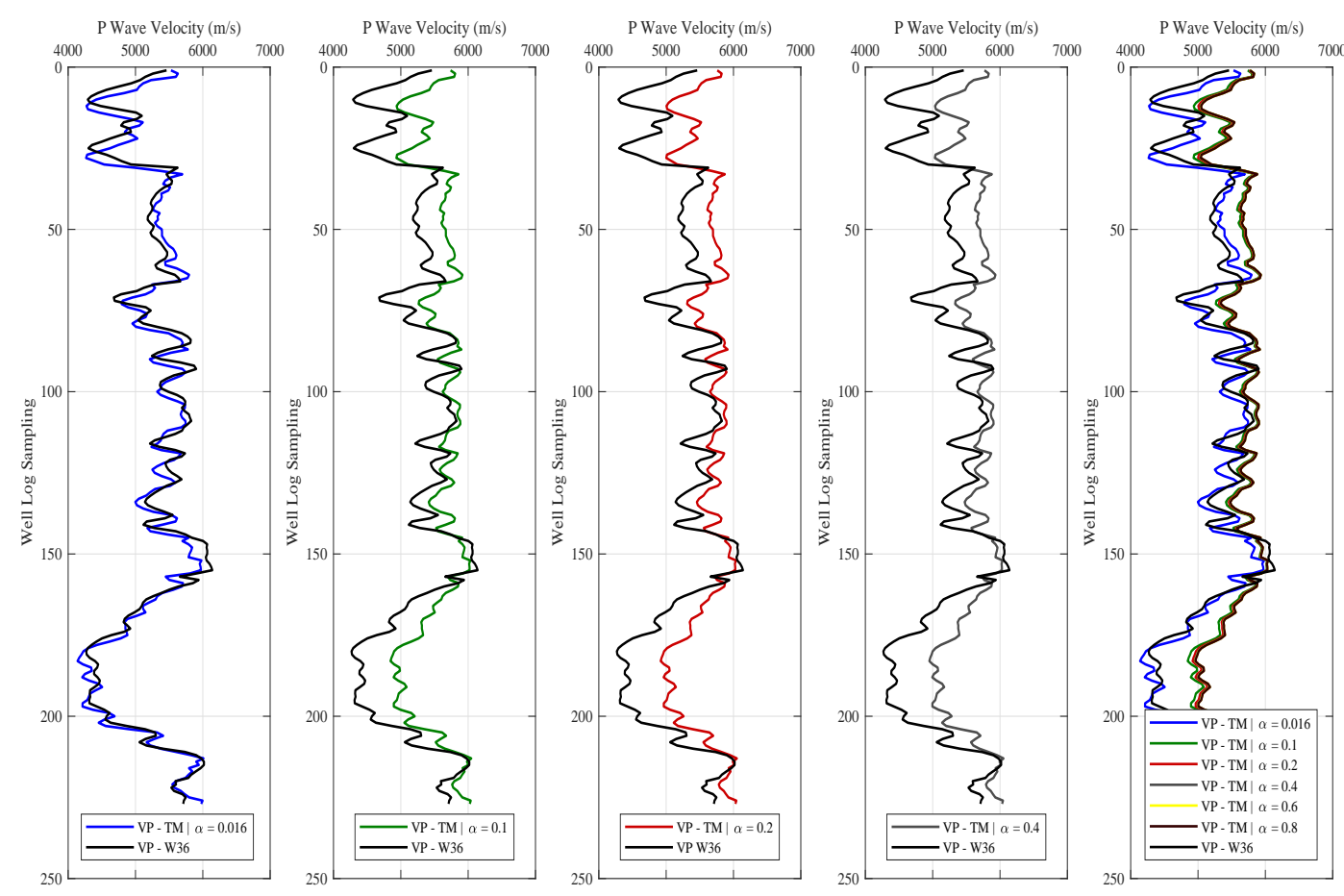

Figure 6.20: Sensitivity analyses regarding different aspect ratios using the rock physics model of T-Matrix with $\alpha=0.016,0.1,0.2,0.4,0.6$ and 0.8 . 


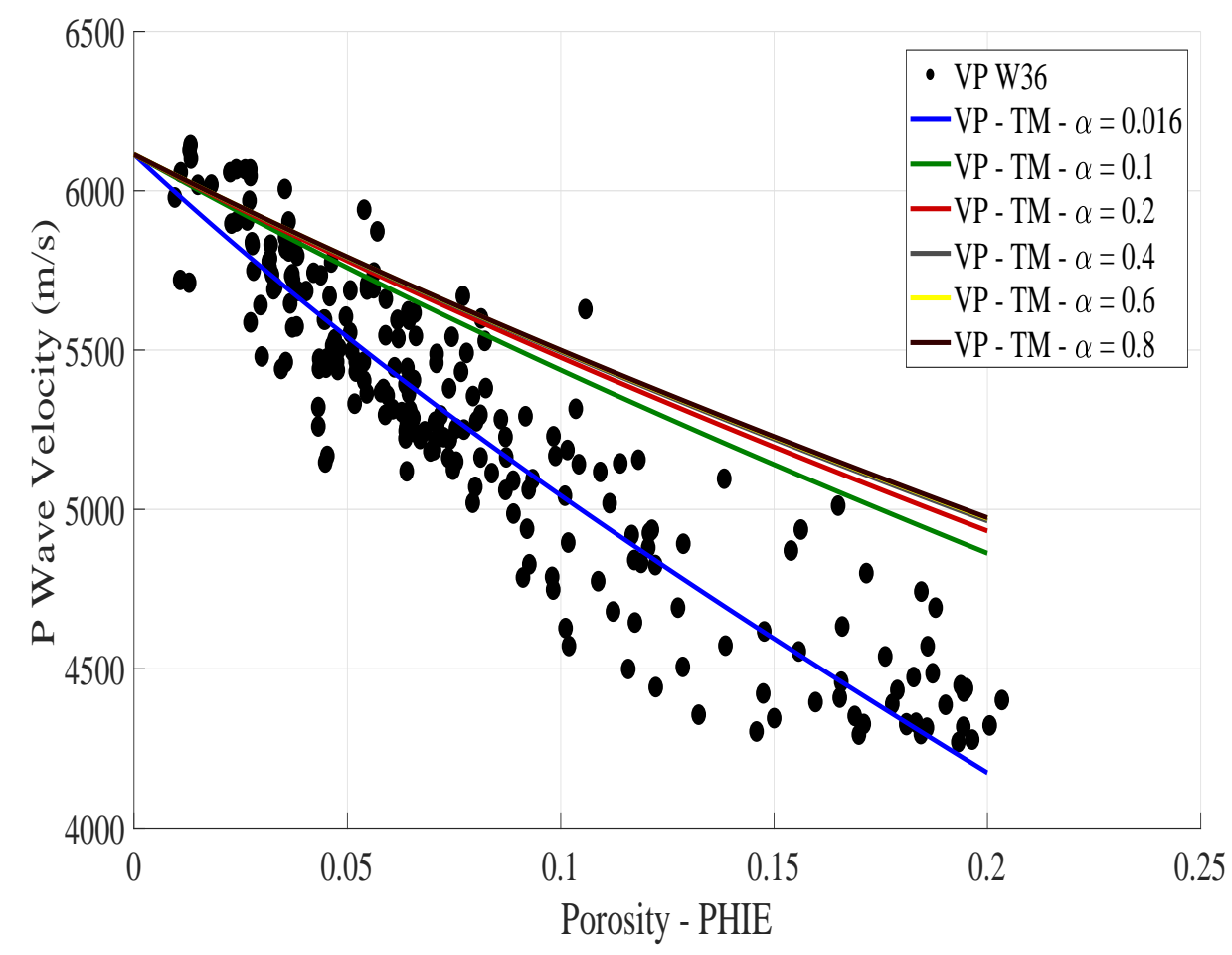

Figure 6.21: Rock physics modelings regarding different aspect ratios using the rock physics model of T-Matrix with $\alpha=0.016,0.1,0.2,0.4,0.6$ and 0.8 over the crossplot $V_{p}-\phi$ of Well 36 .

Some immediate considerations can be quickly obtained through Figures 6.20 and 6.21 , when $5 \%$ of the carbonate reservoir pore space is represented with the following values of aspect ratios: $\alpha=0.016,0.1,0.2,0.4,0.6,0.8$.

- The huge change regarding elastic attributes estimations still happen when $\alpha=0.016$ goes to $\alpha=0.1$.

- It is possible to see the small differences in the rock physics modelings when $\alpha=0.1$ goes to $\alpha=0.2,0.4$.

- The differences in the rock physics modelings estimations when $\alpha=0.4$ goes to $\alpha=0.6$ or $\alpha=0.8$, technically, are almost imperceptible. In this way, there is almost no difference regarding elastic attributes estimations using any of those higher values of aspect ratios.

- The T-Matrix rock physics modelings settled over the crossplot $V_{p}-\phi$ of Well 36 in Figure 6.21 corroborate and illustrate the analyses made in the previous bullets. 
Through Figures 6.18 and 6.20 , it is possible to conclude that the change of $5 \%$ of the reservoir volume proportion from $\alpha=0.016$ to $\alpha=0.1$ makes the reservoir much stiffer, increasing the $\mathrm{P}$ wave velocity, and the changes are very significant. It is very easy to find differences between the first and third tracks in the order $500 \mathrm{~m} / \mathrm{s}$. However, in Figure 6.20, when the aspect ratio goes from $\alpha=0.1$ to higher values, such as: $\alpha=0.2,0.4,0.6$ and 0.8 , some sort of velocity increase happens but it is very modest. It is important to mention again that the big differences were found out for low velocities intervals where the porosities tend to be higher. For high velocities intervals where the porosities tend to be lower, the differences between the modelings using different aspect ratios, tend to be smoother.

Those kind of sensitivity analyses regarding pores with different aspect ratios are especially important for coquinas. In fact, Junior (2016) (43) in his Master's dissertation, approaching coquinas reservoirs, basically stated that this kind of carbonate reservoir with interparticle porosity shows intermediate values of porosity but good values of permeability whereas higher presence of moldic pores indicates good values of porosity but lower values of permeability. Bizotto (2014) (15) follows almost the same conclusions of Junior (2016) (43) and also states that coquinas reservoirs with interparticle pores - more depositional developed - tend to present higher values of porosity and permeability. In this way, for the coquinas reservoir, it is very important to understand the quantitative elastic attributes alterations regarding pore shapes because moldic pores can be modeled with higher values of aspect ratio and the presence of those pores, in this specific carbonate rock, can indicate higher porosities but lower permeabilities.

Certainly, at first glance, there is an intuitive feeling about what happens when the increase of the moldic pores occurs in the rock physics model: the reservoir gets stiffer, in the same way; increasing the crack pores, the reservoir gets softer. However, the goal of using rock physics models is to obtain a quantitative feeling/sense of those changes regarding the aspect ratio variations. In this way, it is possible to better understand quantitatively how the $\mathrm{P}$ wave velocity vary, through the rock physics modeling, altering the input parameters. Nevertheless, as was already mentioned in Chapter 2, the changes in the aspect ratios can be also related to diagenetic processes and other events, as it was described that the moldic pores are related to dissolution and crack pores can be related to fractures. 


\section{4 .2}

\section{Sensitivity Analyses - Fluids}

During this section, it will be possible to understand and analyze in a quantitative manner how different kinds of fluids affect the elastic attributes estimations of the coquinas reservoir through the T-Matrix rock physics model. In this way, Figure 6.22 shows the difference between rock physics modelings with different kinds of fluids. In fact, in the first track, it is possible to see the validated T-Matrix rock physics modeling estimation for Well 36 of Figure 6.7. This estimation was obtained using a fluid mixture where each well log sampling in depth assumed proportional values of fluids bulk modulus and density according to the proportion of oil and brine in the $S_{w}$ well log. This procedure was detailed explained in section 5.2.2.1 in Chapter 5. Therefore, the same model with $\alpha=0.016$ was used to compute the elastic attributes estimations of the carbonate reservoir but instead of using a fluid mixture (Oil/Brine), it was modeled the hypotheses that the rock pore space is totally fulfilled by brine or oil, tracks two and three, in Figure 6.22, respectively.
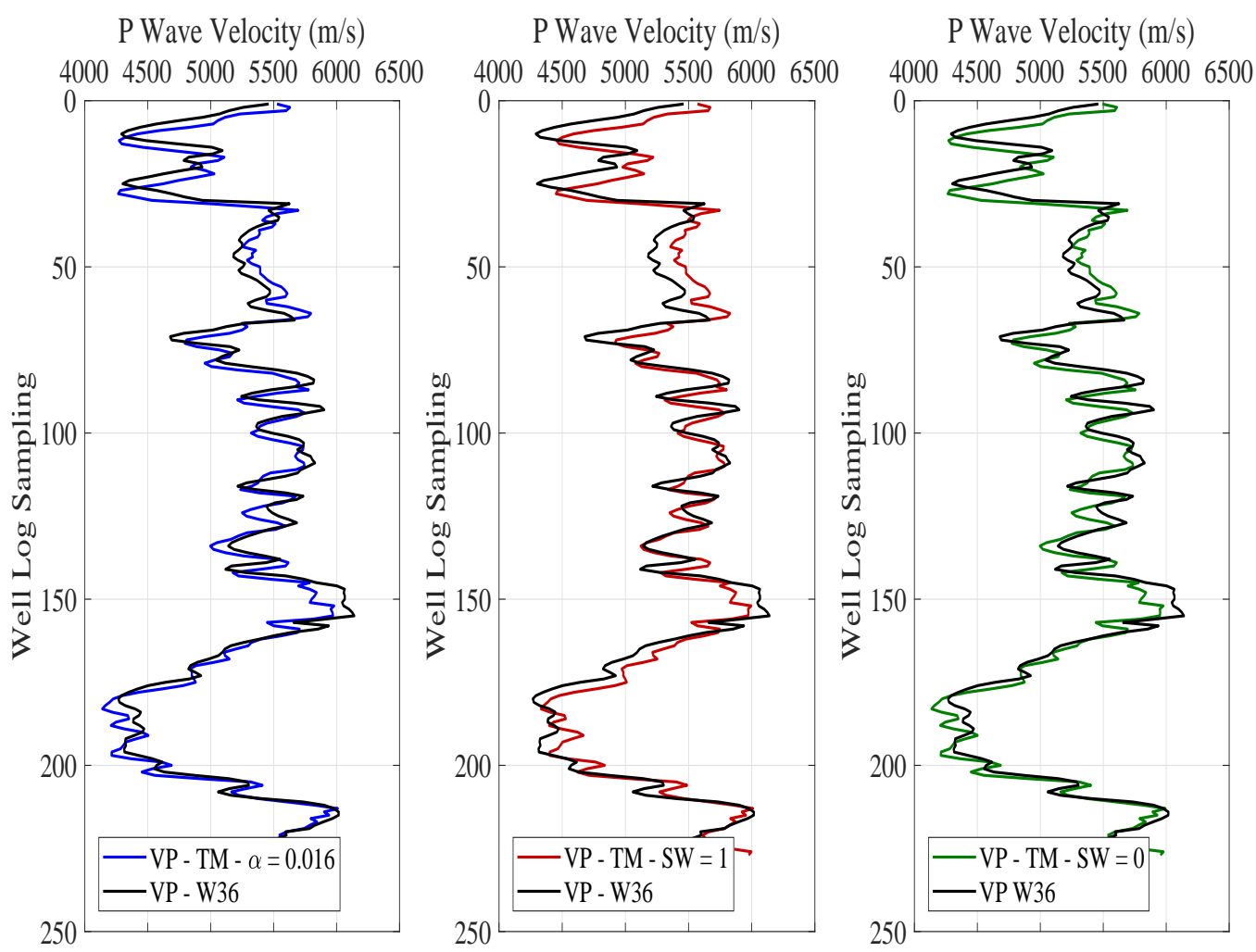

Figure 6.22: Sensitivity Analyses regarding fluids inclusions taking into consideration the hypotheses of all the pores fulfilled by brine (Track 2) and oil (Track 3). 
In this way, through Figure 6.22, in track two, an increase of $\mathrm{P}$ wave velocity can be observed, mainly in the interval of low velocities between samples 150 and 215. On the other hand, in track three of the same figure almost any change can be noticed. In fact, the reason why there is almost no difference between tracks one and three can be illustrated in Figure 6.23.

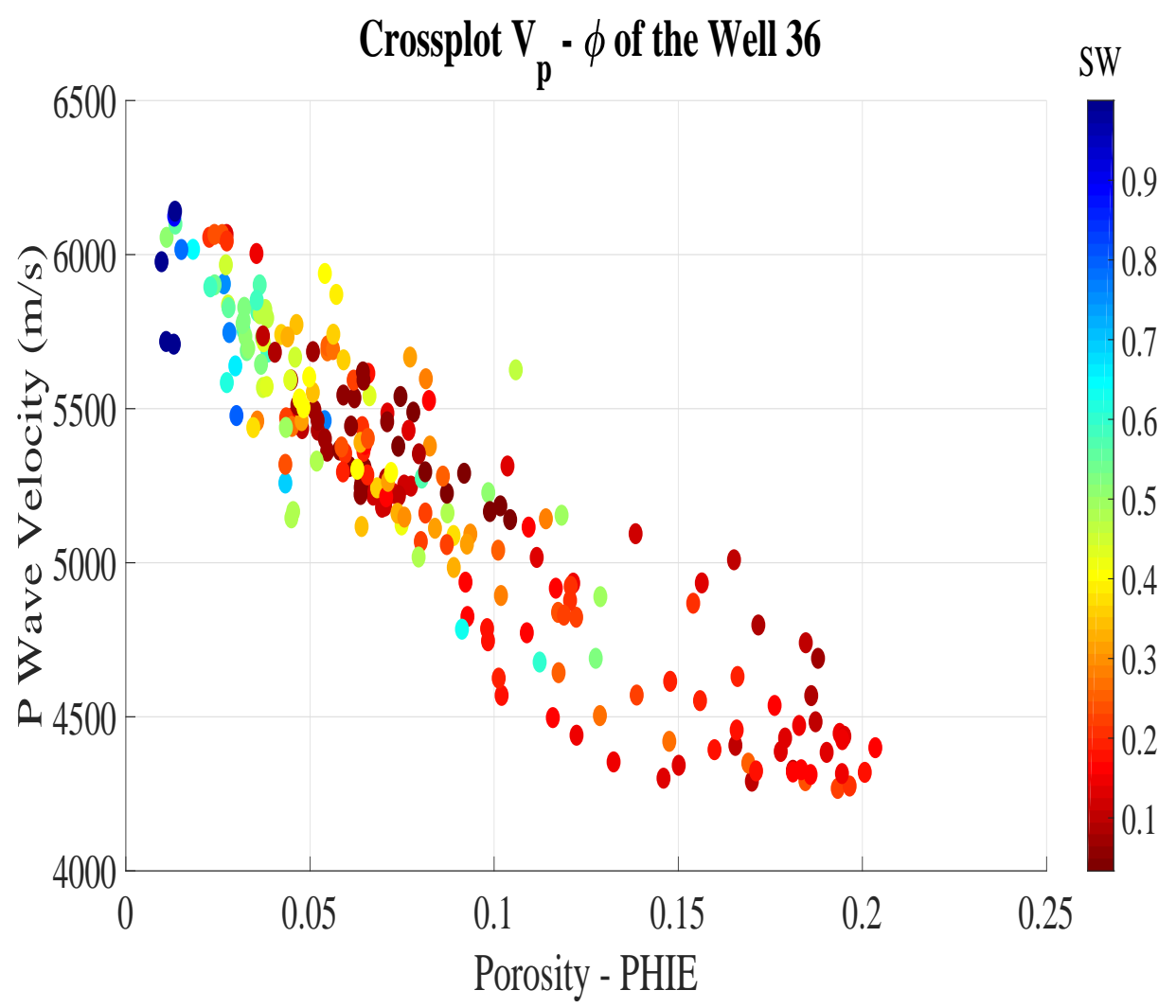

Figure 6.23: Crossplot $V_{p}-\phi$ of the Well 36 colored by the water saturation $\log \left(S_{w}\right)$.

In this figure, it is possible to notice that in this well, the carbonate reservoir is almost fully saturated by oil, mainly for higher values of porosities and consequently, for lower values of velocity. Therefore, it is totally comprehensible that when the fluids mixture is substituted for oil, the difference regarding elastic attributes estimations through the T-Matrix rock physics model will not be relevant after all. In fact, it is possible to better illustrate that the modelings (Validated T-Matrix and the hypothesis of $S_{w}=0$ ) in tracks one and three of Figure 6.22 are very similar in Figures 6.24 and 6.25. In Figure 6.24 , in the second track, it is possible to notice that both plots are superimposed whereas in Figure 6.25, also in the second track, the simulations of both models are also almost superimposed. 

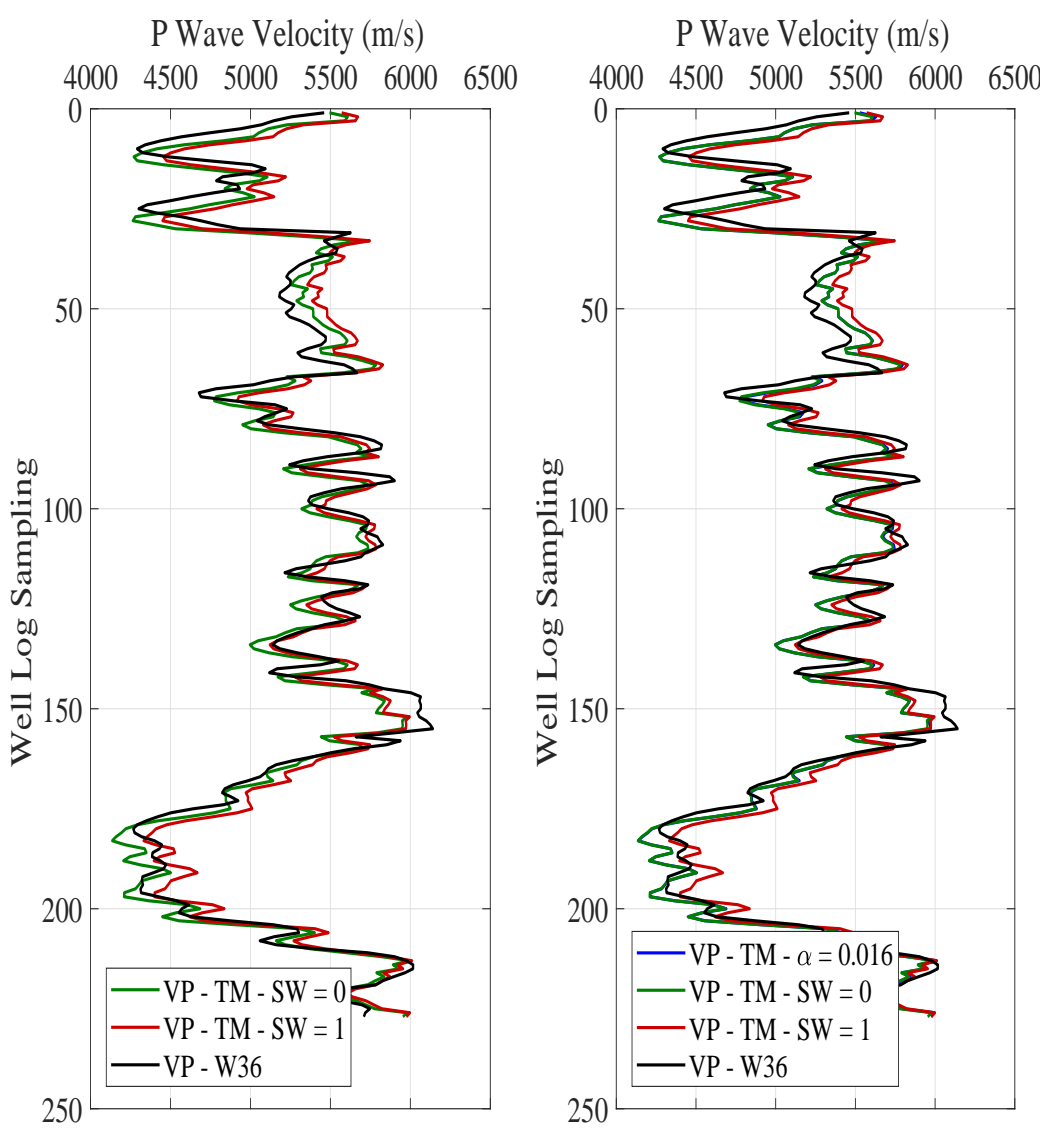

Figure 6.24: Sensitivity Analyses taking into consideration the hypotheses of all the pores fulfilled by brine or oil.
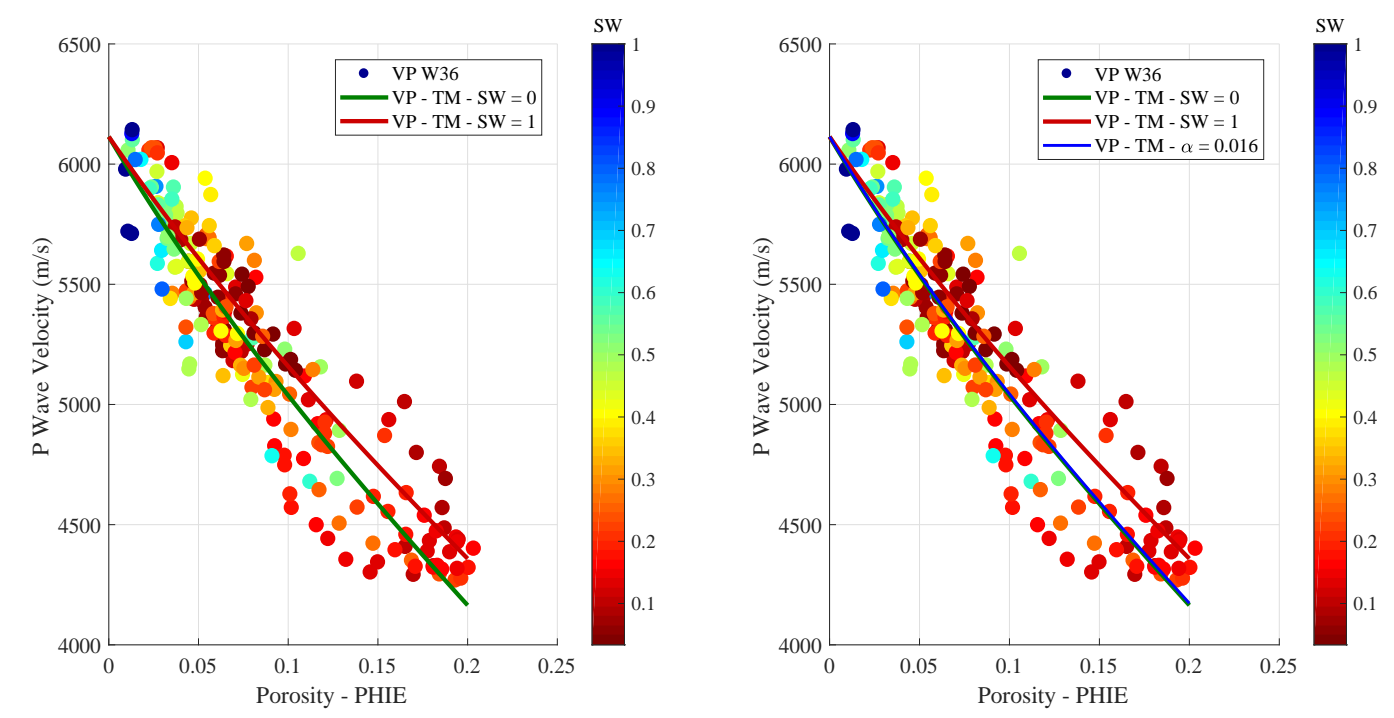

Figure 6.25: Simulations of the T-Matrix rock physics model representing the hypotheses of all the reservoir pores fulfilled by oil $\left(S_{w}=0\right)$ or water $\left(S_{w}=\right.$ 1) and the crossplot $V_{p}-\phi$ colored by the water saturation $\log \left(S_{w}\right)$. 
It is important to mention that all the other parameters were kept constant and the saturating fluid bulk modulus and density were the only parameters changed. In fact, no unusual values of fluid bulk modulus $\left(K_{f l}\right)$ and density $\left(\rho_{f l}\right)$ to run the fluid sensitivity analyses were used. The fluid mixture was composed by the oil bulk modulus $\left(K_{o}\right)$ and density $\left(\rho_{o}\right)$ plus the brine bulk modulus $\left(K_{b}\right)$ and density $\left(\rho_{b}\right)$ following the respective proportions of each fluid in all well log samples along the reservoir column. The only change made was to substitute all the well log samplings with the proportional fluid mixture for constant values of brine bulk modulus or oil bulk modulus $\left(K_{b} \mid K_{o}\right)$ and brine density or oil density $\left(\rho_{b} \mid \rho_{o}\right)$ in the entire reservoir column in each well log sample.

Taking into consideration the first and second tracks of Figure 6.22, it is possible to see a slight increase of $\mathrm{P}$ wave velocity, mainly in the interval of lower velocities between the samples 150 and 215. This interval presents higher porosities and it is almost fully saturated by oil as it can be seen in Figure 6.23. Therefore, running the fluid sensitivity analyses in this interval with brine is a good example to observe the fluids effects on this carbonate reservoir. As a matter of fact, analyzing some samples of this same interval, it was possible to find out differences between the velocities in the order of $200 \mathrm{~m} / \mathrm{s}$ and even 260 $\mathrm{m} / \mathrm{s}$ in the well log sampling 183, comparing tracks 1 and 2 of Figure 6.22. In comparison with the difference of velocities encountered due to the aspect ratio changes in section 6.4.1, those variations are very modest. One of the reasons that may explain why such small differences of velocity were discovered in the fluid sensitivity analyses is because of the intrinsic carbonate stiffness, as was already mentioned in Chapter 2 of this dissertation. In this way, through those analyses, it is possible to verify that this carbonate reservoir tends to be somehow insensitive regarding the fluid changes.

\section{4 .3}

\section{Sensitivity Analyses - Mineralogical Content}

In the two previous sections it was possible to analyze how the $\mathrm{P}$ wave velocity of the coquinas reservoirs behave due to the alterations in the pores aspect ratios and fluids. During this section it will be possible to understand in a quantitative manner how the elastic attributes of the coquinas reservoir behave due to the changes regarding the mineralogical content of the rock matrix. In fact, to be more specific, the bulk and shear moduli ( $K$ and $G$ ) inputs of the T-Matrix rock physics model will be changed in order to simulate three different scenarios. Therefore, the diagenetic processes of dolomitization and low and intense silicification will be modeled taking into consideration the 
information available in the literature about the bulk and shear moduli of the minerals dolomite and quartz.

Those kind of sensitivity analyses are very important because it is not unusual to find out in the literature that the mineralogical content has little effect on the carbonates elastic attributes in comparison with other properties, such as: porosity or pores aspect ratios. The dolomitization and silicification processes were chosen to be modeled in this section because they are some of the most common diagenetic processes which affect the carbonate rocks - in a general manner - together with processes of cementation and dissolution. In fact, the diagenetic process of dissolution was already simulated in section 6.4.1 where it was possible to analyze the increase of $\mathrm{P}$ wave velocity when the pores aspect ratios increased, leading the reservoir to get much stiffer. Besides that, Junior (2016) (43) and Bizotto (2014) (15) pointed out that the diagenetic processes of dolomitization and silicification are common in coquinas reservoirs. Nevertheless, Figure 6.26 illustrate how the $\mathrm{P}$ wave velocities and $V_{p} / V_{s}$ ratios of the coquinas reservoir change due to three different scenarios: dolomitization, low silicification and intense silicification.
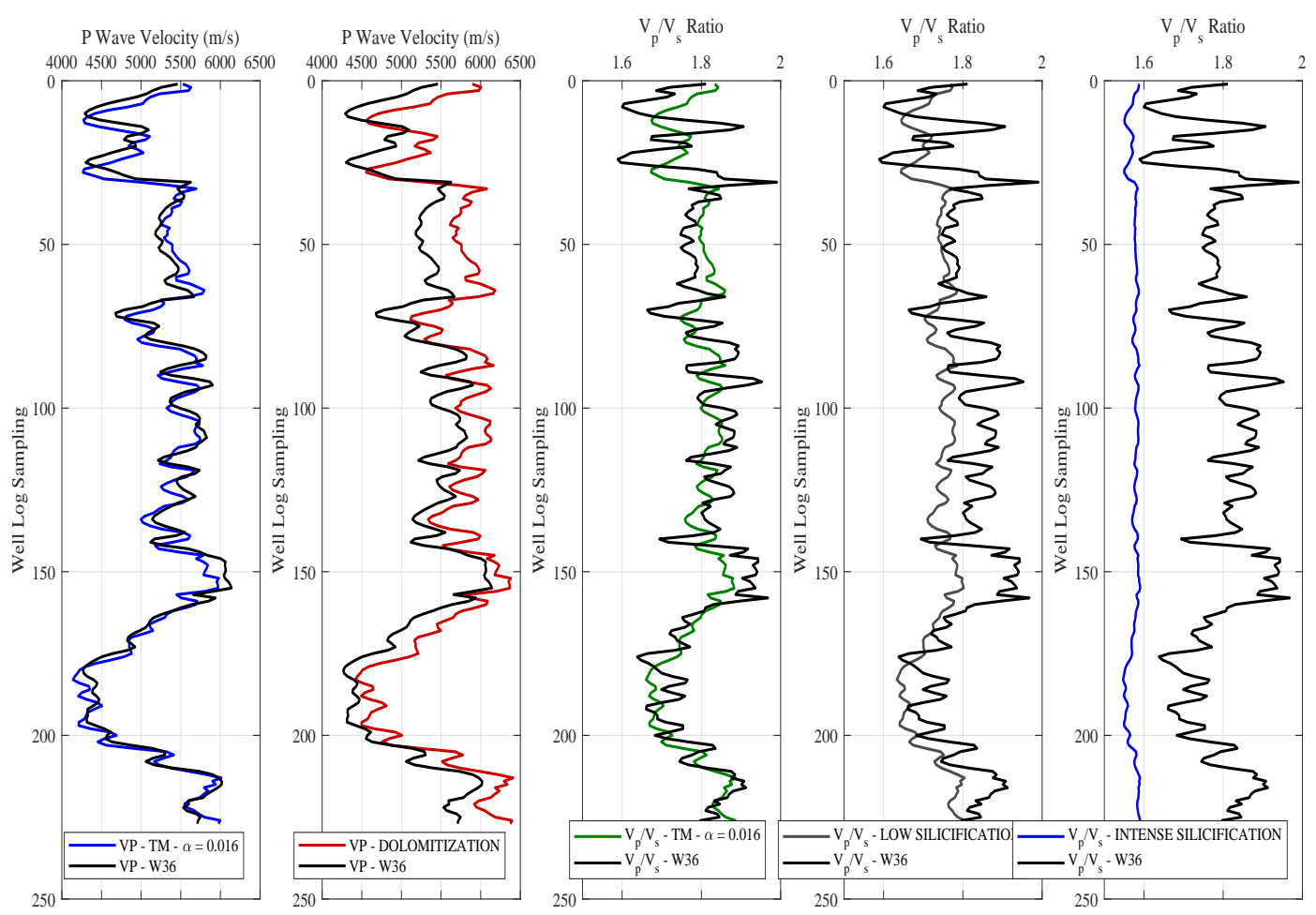

Figure 6.26: Sensitivity analyses regarding different values of bulk and shear moduli using the T-Matrix rock physics model. 
In the first track of Figure 6.26, it is possible to observe the T-Matrix rock physics model parametrized with the optimized parameters generated through the DREAM algorithm using the Bayesian framework where the matrix $100 \%$ composed by the mineral calcite (validated T-Matrix) was considered. In the second track, it is clear to notice the increase of the $\mathrm{P}$ wave velocity in the carbonate reservoir taking into consideration that the reservoir matrix is composed by the following proportions: $65 \%$ calcite $-35 \%$ dolomite (dolomitization scenario). The calcite bulk and shear moduli were extracted from Table 6.2 and those values can also be seen in Table 6.9 whereas the dolomite bulk and shear moduli were also taken from Table 6.9. The dolomite elastic moduli were chosen among the three references available in the book of Mavko et al., (2009) (68).

The Voigt and Reuss bounds together with the mixing law of Hill were applied in order to obtain the resultant bulk and shear elastic moduli of the new matrix of the carbonate reservoir which will be simulated. The new values of K, G (bulk and shear moduli) and $\rho$ (density) of the new matrix can be analyzed in Table 6.10 (dolomitization scenario). Regarding the density, the Voigt's average was used to obtain the resultant density of the new elastic solid media. In fact, in Figure 6.26, it is very easy to discover differences between the first and the second tracks in the order of $300 \mathrm{~m} / \mathrm{s}$ for intervals of lower velocities and higher porosities whereas in intervals of higher velocities and lower porosities, such as: between the well log samplings 25 and 75, differences of $V_{p}$ in the order of $500 \mathrm{~m} / \mathrm{s}$ were found out. Thus, through this modeling, it was possible to see in a quantitative manner how the $\mathrm{P}$ wave velocity of this carbonate reservoir behaves when a sort of dolomitization happens in the coquinas reservoir.

It is important to mention that is not intuitive to evaluate sensitivity analyses of the diagenetic processes of low and intense silicification analyzing the variations of $\mathrm{P}$ wave velocities. In fact, when $V_{p} / V_{s}$ is used instead of $V_{p}$ in order to make such an evaluation, it is possible to cut the effect of density and leverage the effects bulk and shear moduli. Therefore, through $V_{p} / V_{s}$ analysis, it is possible to have a more clear evaluation without any ambiguities. Despiting the fact that we did not have the DTS logs available of the three wells studied in this dissertation and taking into consideration good estimations of $V_{s}$ values for the core samples, the $V_{p} / V_{s}$ estimations of the third track of Figure 6.26 (black solid line) were estimated using the real $V_{p}$ well log from Well 36 and the $V_{s}$ estimations generated through the T-Matrix rock physics model. Likewise, in the same track, it is possible to notice the estimations of the parametrized and calibrated T-Matrix rock physics model (green solid line). Nevertheless, in 
the fourth and fifth track of Figure 6.26, it is possible to analyze how the elastic attributes of the coquinas reservoir behave taking into account the scenarios of low and intense silicifications.

The same procedure addressed to simulate the diagenetic process of dolomitization was approached to simulate the hypotheses of low and intense silicifications using the following mineralogical content proportions, respectively: 85\% Calcite - 15\% Quartz and 35\% Calcite - 65\% Quartz. The bulk and shear moduli of the minerals calcite and quartz were extracted from Table 6.9. In fact, the values of K, G (bulk and shear moduli) and $\rho$ (density) of the new matrix regarding the diagenetic processes of low and intense silicification can be analyzed in Table 6.10. In this way, in Figure 6.26, it is possible to observe that for the scenario of low silicification, the $V_{p} / V_{s}$ ratio differences between the third and fourth tracks is not too expressive (in the order of 0.1 ). On the other hand, taking into consideration the scenario of intense silification, it is possible to notice expressive variations comparing the $V_{p} / V_{s}$ ratio estimations of the third and fifth tracks (in the order of 0.3 ).

Table 6.9: Values of Shear and Bulk moduli of Calcite, Dolomite and Quartz Minerals.

\begin{tabular}{|c|c|c|c|}
\hline Mineral & Bulk Modulus (GPa) & Shear Modulus (GPa) & Density $\left(\mathrm{Kg} / \mathrm{m}^{3}\right)$ \\
\hline Calcite & 63,7 & 28,2 & 2,710 \\
\hline Dolomite & 94,9 & 45 & 2,870 \\
\hline Quartz & 37 & 44 & 2,650 \\
\hline
\end{tabular}

Dolomite and quartz elastic moduli extracted from Mavko et al., (2009) (68).

Table 6.10: Bulk and Shear Moduli obtained using the Voigt-Reuss averages together with the Mixing Law of Hill and Density obtained through the Voigt's average.

Diagenetic Bulk Modulus (GPa) Shear Modulus (GPa) Density (Kg/m³) Process

\begin{tabular}{lccc}
\hline Dolomitization & 73,3 & 33,2 & 2.766 \\
$\begin{array}{l}\text { Low Silicifi- } \\
\text { cation }\end{array}$ & 58,6 & 30,1 & 2.701 \\
$\begin{array}{l}\text { Intense Sili- } \\
\text { cification }\end{array}$ & 44,8 & 37,6 & 2.671
\end{tabular}




\section{7}

\section{Conclusions, Final Remarks and Future Work}

It is hard to believe how granular pack rock physics models - such simplistic models - can represent siliciclastic reservoirs elastic attributes, as Dvorkin et al., 2014 (30) has already mentioned in their book. Following the same perspective, this work was conducted: how can a rock physics model embedded with so many assumptions, idealizations and limitations estimate elastic attributes of carbonates? Particularly, coquinas, such a heterogeneous reservoir rock, composed by so much debris, organism shelfs and geological components all mixed and unified in a single geological structure that we gave the name of reservoir rock. Therefore, since the beginning of this research, we knew that it would be a challenge to accomplish such a task with a low degree of error, due to all the heterogeneities embedded in the coquinas reservoirs and carbonates in a general manner. In fact, that was one of the reasons why some input parameters of the rock physics models were posed in a Bayesian framework. Through this approach, it was possible to stochastically evaluate the main parameters of those models and take into consideration inherent uncertainties related to the measurements needed as inputs. In this way, it was possible to reach more reliable elastic attributes estimations from both models.

Rock physics is not a new research field but some applications just came up in the recent years and during this time, the industry has been recognizing the applicability of rock physics as a whole in exploratory process, mainly, due to the good results obtained in the literature, in several aspects, such as: model driven seismic inversions, pore type characterizations, synthetics generation through rock physics models, among others. This dissertation is just one more little contribution in the scope of Rock Physics which shows the application of those models in order to reliably estimate elastic attributes of carbonate reservoirs. Moreover, it was also demonstrated - in a quantitative manner - how the coquinas elastic attributes behave regarding the changes in pores aspect ratios, mineralogical content and fluids through sensitivity analyses. In this way, taking into consideration the scope of this work and the results achieved during this dissertation, the following conclusions were obtained: 
1. Regarding well logs, it was possible to reach through the rock physics models of $\mathrm{Xu} \&$ Payne and T-Matrix accurate elastic attributes estimations for Wells 49, 36 and 10. However, it is important to mention that the $\mathrm{Xu} \&$ Payne approach generated picks of underestimation in Wells 49 and 36 for intervals of lower velocities and higher porosities. In this way, for a more complete evaluation of the models performance regarding elastic attributes estimations, the analyses of the metrics of accuracy (MAE and RMSE) and mainly the error histograms were also necessary, where it was possible to observe not only the most frequent errors but also the maximum errors obtained between the modeled and measured $V_{p}$ for each well. Therefore, it was possible to conclude that in a general manner the T-Matrix had a better performance in the matter of elastic attributes estimations over the rock physics model of $\mathrm{Xu} \&$ Payne regarding well log data.

2. In respect of the elastic attributes estimations of the T-Matrix rock physics model using the well log data, it is possible to state that this model was well parametrized in the well location (Well 49) following the metrics of accuracy and the histograms between the modeled and the measured $V_{p}$. Besides that, the same calibrated model was extrapolated and validated for Wells 36 and 10, where most of the errors presented low percentage values and good metrics of accuracy were also obtained. In this way, it was possible to conclude that the calibrated T-Matrix rock physics model not only had a good performance and it was well calibrated at the well location (Well 49), but also had a good power of extrapolation estimating the elastic attributes with accuracy for other wells (Wells 36 and 10) which were distant from the calibration well. In fact, the parameters evaluation through the Bayesian framework was applied with the data of Well 49, which is the calibration well.

3. The MAP values of the optimized posterior parameters distributions obtained through the Bayesian framework using well log data were also used in order to estimate the elastic attributes of carbonate core samples. Therefore, it was possible to estimate not only $\mathrm{P}$ wave velocities $\left(V_{p}\right)$ but also $\mathrm{S}$ wave velocities $\left(V_{s}\right)$ and $V_{p} / V_{s}$ ratios where reliable estimations were obtained with good metrics of accuracy through the rock physics models of Xu \& Payne and T-Matrix. In a general manner the T-Matrix 
demonstrated to be superior over the rock physics model of Xu \& Payne regarding reliable elastic attributes estimations. In fact, this result is very interesting because it was possible to verify that optimizing the parameters through the Bayesian framework only using $V_{p}$ data, it was possible to reliably estimate $\mathrm{S}$ wave velocities $\left(V_{s}\right)$ and $V_{p} / V_{s}$ ratios for the core samples.

4. In the conclusions 1 and 3, it was stated that the T-Matrix rock physics demonstrated - in a general manner - to be superior than the Xu \& Payne approach regarding reliable elastic attributes estimations. Both statements were made in this manner because in the $V_{p}$ estimations of Well 10 and in the $V_{s}$ estimations of the core samples, following the metrics of accuracy (RMSE, MAE and Mean Error), the Xu \& Payne proved to be slightly better than the T-Matrix, technically almost equal regarding some metrics of accuracy and histograms analyses. On the other hand, in the $V_{p}$ estimations of Wells 49 and 36 and in the $V_{p}$ and $V_{p} / V_{s}$ estimations of the core samples, following the metrics of accuracy and histograms, the T-Matrix demonstrated to present much better elastic attributes estimations in comparison to the Xu \& Payne model. That is why in a general manner the T-Matrix proved to be superior over the $\mathrm{Xu} \&$ Payne rock physics model regarding well logs and core samples data.

5. Through the calibration of the T-Matrix rock physics model at the well location and the accurate elastic attributes estimations regarding other wells and core samples - validation -, it was possible to state that this model can represent the elastic attributes of the carbonate reservoir which has been studied in this dissertation. Taking into consideration those premises, it was possible to use the T-Matrix model in order to quantitatively understand how the carbonate elastic attributes behave regarding the changes of aspect ratios, fluids and mineralogical content. Those are the so-called sensitivity analyses and the following conclusions were obtained in this regard:

- It was possible to conclude that the model is very sensitive regarding aspect ratios, mainly when $\alpha=0.016$ goes to $\alpha=0.01$ or $\alpha=0.1$ where velocities variations in the order of $500-600 \mathrm{~m} / \mathrm{s}$ were found out. However, when the aspect ratio $\alpha=0.2$ goes to $\alpha=0.4,0.6$ or $\alpha=0.8$, the model tends to be insensitive where 
the changes of velocity are almost imperceptible. In fact, those simulations were made taking into consideration the change of only $5 \%$ of the total pore space of the coquinas reservoir.

- During the sensitivity analyses regarding the fluids, it was possible to verify that this carbonate reservoir tends to be somehow insensitive due to the fluid changes. In fact, variations in the order of 200 $\mathrm{m} / \mathrm{s}$ even $260 \mathrm{~m} / \mathrm{s}$ were found out simulating the brine hypothesis. Those variations in comparison to the variations encountered due to the aspect ratio changes are very modest.

- The sensitivity analyses regarding the mineralogical content showed that the dolomitization and intense silicification diagenetic processes tend to be more prominent in comparison to the low silicification process. In fact, velocity variations in the order of $300 \mathrm{~m} / \mathrm{s}$ were discovered for porous intervals, whereas variations approximately of $500 \mathrm{~m} / \mathrm{s}$ for intervals with lower porosities were found out. Those simulations were computed taking into consideration that the rock matrix assumed the following values of mineralogical content: $65 \%$ calcite and $35 \%$ dolomite. However, in the hypothesis of low silicification, there were not too expressive changes regarding elastic attributes estimations whereas with respect of intense silicification variations in the order of 0.3 were discovered showing strong variations of $V_{p} / V_{s}$ ratios.

In respect to the fifth conclusion, it was possible to verify that the TMatrix rock physics model has more sensibility regarding the aspect ratios and mineralogical changes. Regarding the aspect ratio simulations, this conclusion corroborates what the literature has been stating through the years: the main factors which affect the carbonate elastic attributes are porosity and pore aspect ratios. On the other hand, it is very easy to see in the literature that the mineralogical content of carbonates has little effect in terms of those reservoir elastic attributes. Following our conclusions, it was possible to verify that taking into consideration the hypothesis of dolomitization, differences of $V_{p}$ in the order of $300 \mathrm{~m} / \mathrm{s}$ were achieved for porous intervals whereas for intervals with lower porosities, $\mathrm{P}$ wave velocities variations in the order of 500 $\mathrm{m} / \mathrm{s}$ were found out. Likewise, simulating the intense silicification diagenetic process, it was also possible to verify significant changes in the $V_{p} / V_{s}$ ratios. In this way, it is possible to conclude that the mineralogical content also has an expressive contribution in order to alter the carbonate (coquinas) 
elastic attributes despite what has been stated in the literature. Moreover, this carbonate reservoir demonstrated to be less sensitive to the fluid alterations even for intervals of higher porosities. This conclusion also corroborates the information of the literature which states that due to the carbonate stiffness, this kind of rock tends to be insensitive regarding fluid alterations.

It is important to mention that the rock physics modelings conducted during this dissertation were applied in order to estimate the elastic attributes of a specific carbonate reservoir: coquinas. Therefore, the validity of this work and the models approached herein are related to the reservoir which was studied during this dissertation. Taking into consideration the wide variety of carbonate rocks and their different facies, frameworks, pore shapes and also the different possible diagenetic processes that may happen, those calibrated models are not likely to be applicable for other carbonate rocks with different faciological and sedimentological characteristics. In fact, we do believe that for other carbonate rocks, the rock physics models must be calibrated, parametrized and validated taking into account new information that must be acquired which will disclose about the specific features of each carbonate rock, hence, those characteristics can be embedded in the models in order to estimate the reservoir elastic attributes. Thus, the conclusions obtained during this work regarding the rock physics modelings and the sensitivity analyses for coquinas reservoirs are not likely to be extrapolated for other carbonate reservoirs with different sedimentological and faciological features.

Certainly, a lot of subjects inside of the Rock Physics scope have been developed and much more work needs to be done in the years to come. I believe that in the coming years, rock physics awareness will increase even more and professionals will still find manners to embed this research field not only in the exploration process - helping to find the hard oil, because the easy one is vanishing - but also in the development processes. In recent years, some techniques related to the machine learning scope have been shown in literature, in the matter of reservoir characterization, elastic attributes estimations and others. In fact, those approaches came to help and in some specific areas, such as: seismic interpretation, those algorithms related to neural networks, support vector machines, genetic algorithms, image processing and deep learning will really become paramount regarding the increase of seismic interpreters productivity. However, taking into consideration the reservoir characterization scope, only those algorithms working in a data-driven approach, probably will not solve the issues of extracting petrophysical properties of heterogeneous reservoirs reliably. Nevertheless, the coupled approach of data-driven and model-driven - related to rock physics foundations - techniques can be a trend 
in future years to solve these kinds of problems related to the reliable extraction of petrophysical properties and elastic attributes from heterogeneous reservoirs using seismic and well logs as inputs.

\section{1}

\section{Future Work}

The following topics can be pointed out as future work or the continuation of this dissertation:

- Generation of synthetics using an extracted wavelet and analyze in a quantitative manner how the amplitudes behave regarding the changes in the input parameters, such as: mineralogical content, fluids and pores aspect ratios.

- Conduct wider sensitivity analyses through the calibrated and validated rock physics model. In this way, combine different alterations in the model inputs at the same time in order to simulate more consistent diagenetic processes and geological events in the carbonate reservoir.

- Explore more the potential of the T-Matrix rock physics model, such as: rock physics modelings regarding anisotropy.

- Through both rock physics models understand in a quantative manner the main factors which drive the AVO signatures in carbonate rocks. 


\section{Bibliography}

[] ANSELMETTI, F.; EBERLI, G.. Controls on sonic velocity in carbonates. In: PAGEOPH. 141, p. 287-323, 1993.

[1] ANSELMETTI, F. S.; EBERLI, G. P.. Sonic velocity in carbonate sediments and rocks. Geophysical Development Series, 1997.

[2] AHR, W. M.. Geology of Carbonate Reservoirs: The Identification, Description, and Characterization of Hydrocarbon Reservoirs in Carbonate Rocks. Wiley, 2008.

[3] ABREU, E. S.. Modelagens teóricas e empíricas aplicada à investigação da conexão entre as propriedades petrofísicas e elásticas em rochas carbonáticas. Master's thesis, UNICAMP, 2010.

[4] AgERSBORG, R.; JOHANSEN, T. A. ; JAKOBSEN, M.. The t-matrix approach for carbonate rocks. In: SEG ANNUAL MEETING, 2005.

[5] AGERSBORG, R.; JOHANSEN, T. A. ; JAKOBSEN, M.. Velocity variations in carbonate rocks due to dual porosity and wave-induced fluid flow. In: GEOPHYSICAL PROSPECTING, 2009, 57, p. 81-98, 2009.

[6] AGESBORG, R.. Seismic properties of carbonate rocks with emphasis on effects of the pore structure. $\mathrm{PhD}$ thesis, University of Bergen, 2007.

[7] AGERSBORG, R.; JOHANSEN, T. A.; JAKOBSEN, M.; SOTHCOTT, J. ; BEST, A.. Effects of fluids and dual-pore systems on pressuredependent velocities and attenuations in carbonates. In: GEOPHYSICS,VOL. 73, NO. 5, p. N35-N47, 2008.

[9] ANSELMETTI, F.; EBERLI, G.. The velocity deviation log: A tool to predict pore type and permeability trends in carbonate drill holes from sonic and porosity or density logs. In: AAPG BULLETIN, V.83, NO.3, p. 450-466, 1999.

[10] ARTOLA, F. V.; VILLALOBOS, J. H.; MATHEWSON, J.; F.A. ALVAREZ AND, C. S.; CAMACHO, R.; OLARTE, F. O. ; ALPIREZ, L.. Diagenetic considerations and geophysical poretype characterization 
in ts carbonate reservoirs. In: THIRD EAGE/SBGF WORKSHOP 2016, 2016.

[11] AVSETH, P. A.. Combining Rock Physics and Sedimentology for Seismic Reservoir Characterization of North Sea Turbidite Systems. PhD thesis, Stanford University, 2000.

[12] AVSETH, P.; MUKERJI, T. ; MAVKO, G.. Quantitative Seismic Interpretation: Applying Rock Physics Tools to Reduce Interpretation Risk. Cambridge, first edition, 2005.

[13] AVSETH, P.; CARCIONE, J.. Rock-physics analysis of clay-rich source rocks on the norwegian shelf. The Leading Edge, 2015.

[14] ALLO, F.; COLEOU, T.; COLNARD, O.; MACHECLER, I.; DILLON, L.; NETO, G. S.; NUNES, C. ; DE ABREU, E.. Petrophysical seismic inversion over a carbonate field offshore brazil. 2011.

[15] BIZOTTO, A. C.. Eoaptians coquinas from southwest region of campos basin: Deposicional facies, diagenetic events and control factor of the reservoir quality. Master's thesis, UNESP, 2014.

[16] BAECHLE, G. T.; WEGER, R. J.; EBERLI, G. P. ; MASAFERRO, J. L.. The role of macroporosity and microporosity in constraining uncertainties and in relating velocity to permeability in carbonate rocks. SEG 74th Annual Meeting, 2004.

[17] BATZLE, M.; WANG, Z.. Seismic properties of pore fluids. Geophysics, 1992.

[18] BERRYMAN, J.. Long-wavelength Propagation in Composite Elastic Media II. Elipsoidal Inclusions, volumen 6. J. Acoust. Soc. Am., dec 1980.

[19] BERRYMAN, J.. Single-scattering approximations for coefficients in biot's equations of poroelasticity. The Journal of the Acoustical Society of America, 96:1-23, 1992.

[20] LOURES, L. G.; MORAES, F.. Bayesian porosity inference using rock physics and geostatitical modeling. CSEG Geophysics, 2002.

[21] DO CARMO LUCCHESI LOURES, L. G.. Inferência de Porosidade em Reservatório de Petróleo a partir de Múltiplos Dados de Poço e Superfície. PhD thesis, UENF, 2001. 
[22] SCHARNAGL, B.; VRUGT, J.; VEREECKEN, H. ; HERBST, M.. Inverse modeling of in situ soil water dynamics: investigating the effect of different prior distributions of the soil hydraulic parameters. Hydrology and Earth System Sciences, 2011.

[23] SCHARNAGL, B.; VRUGT, J.; VEREECKEN, H. ; HERBST, M.. Bayesian inverse modeling of soil water dynamics at the field scale: using prior information about the soil hydraulic properties. Hydrology and Earth System Sciences, 15, 2011.

[24] CHIBURIS, E.; LEANEY, S.; SKIDMORE, C.; FRANCK, C. ; MCHUGO, S.. Hydrocarbon detection with avo. In: SEISMICS - OILFIELD REVIEW, 1993.

[25] CHOQUETTE, P.; PRAY, L.. Geologic nomenclature and classification of porosity in sedimentary carbonates. In: AAPG BULLETIN 54, p. 207-250, 1970.

[26] COLEOU, T.; ROUSTIAU, A.; MACHECLER, I.; AYZENBERG, M.; FAYEMENDY, C.; SKJEI, N.; GJERDING, K.; THEUNE, U.; SKJERVHEIM, J.-A. ; ZACHARIASSEN, E.. 4d petrophysical seismic inversion: case studies. In: 75TH EAGE CONFERENCE \& EXHIBITION INCORPORATING SPE EUROPEC 2013, 2013.

[27] COLEOU, T.; ALLO, F.; COLNARD, O.; MACHECLER, I.; DILLON, L.; SCHWEDERSKY, G.; NUNES, C.; ABREU, E. D.; COLPAERT, A. ; VAN WIJNGAARDEN, A.. Petrophysical seismic inversion over an offshore carbonate field. In: 74TH EAGE CONFERENCE \& EXHIBITION INCORPORATING SPE EUROPEC 2012, 2012.

[28] CODO, J.; FOURNIER, F.. Rock physics modeling of diagenetic processes of pre-salt carbonates. In: THIRD EAGE/SBGF WORKSHOP 2016, 2016.

[29] Cersosimo, D. S.; RaVAZZOLI, C. L. ; MARTíneZ, R. G.. Prediction of lateral variations in reservoir properties throughout an interpreted seismic horizon using an artificial neural network. The Leading Edge, 35(3):265-269, 2016.

[30] DVORKIN, J.; GUTIERREZ, M. ; GRANA, D.. Seismic Reflections of Rock Properties. Cambridge, 2014. 
[31] DVORKIN, J.; NUR, A.. Elasticity of high-porosity sandstones: Theory for two north sea data sets. In: GEOPHYSICS, 61, p. 13631370, 1996.

[32] DRAEGE, A.. Rock physics diagnosis - how far can we go? an approach at the edge. In: SEG TECHNICAL PROGRAM EXPANDED ABSTRACTS 2012, p. 1-5, 2012.

[33] VRUGT, J. A.; TER BRAAK, C.; C.G.H. DIKS, D. H.; ROBINSON, B. ; HYMAN, J.. Accelerating markov chain monte carlo simulation by differential evolution with self-adaptive randomized subspace sampling. International Journal of Nonlinear Sciences and Numerical Simulation, 10, 2009a.

[34] DUNHAM, R. J.. Classification of carbonate rocks according to depositional texture. In: AMERICAN ASSOCIATION OF PETROLEUM GEOLOGISTS, p. 108-122, Tulsa, 1962.

[35] VRUGT, J. A.; TER BRAAK, C.; CLARK, M.; HYMAN, J. ; ROBINSON, B.. Treatment of input uncertainty in hydrologic modeling: Doing hydrology backward with markov chain monte carlo simulation. Water Resources Research, 44, 2008a.

[36] ElBAZ, M.; AL-ANAZI, B. D. ; ARVANITIS, M.. Avo analysis in carbonate reservoirs. In: SAUDI ARABIA OIL AND GAS, 2010.

[37] EBERLI, G. P.; BAECHLE, G. T.; ANSELMETTI, F. S. ; INCZE, M. L.. Factors controlling elastic properties in carbonate sediments and rocks. In: THE LEADING EDGE, 22, p. 654-660, 2003.

[38] EMBRY, A.; KLOVAN, J.. A late devonian reef tract on northeastern banks island. In: CANADIAN PETROLEUM GEOLOGY BULLETIN, V. 19, p. 730-781, 1971.

[39] FOLK, R.. Practical petrographic classification of limestones. In: AMERICAN ASSOCIATION OF PETROLEUM GEOLOGISTS BULLETIN, V. 43, p. 1-38, 1959.

[40] FOLK, R.. Spectral subdivision of limestone types. In: AMERICAN ASSOCIATION OF PETROLEUM GEOLOGISTS, p. 62-84, 1962.

[41] FAHIMUDDIN, A.. Petro-elastic modeling of a north sea reservoir: Rock physics recipe and eclipse simulator, 2009. 
[42] GASSMANN, F.. Über die elastizität poroöser medien. Vier. Der Natur. Gesellschaf in Zürich, 96:1-23, 1951.

[43] JUNIOR, R. H.. Controles deposicionais e diagenéticos das propriedades petrofísicas dos reservatórios aptianos/barremianos do grupo lagoa feia no norte da bacia de campos. Master's thesis, UFRGS, 2016.

[44] GUO, Z.; LI, X.-Y.; LIU, C.; FENG, X. ; SHEN, Y.. A shale rock physics model for analysis of brittleness index, mineralogy and porosity in the barnett shale. Journal of Geophysics and Engineering, 2013.

[45] VASQUEZ, G.; DILLON, L.. Módulos adiabáticos e isotérmicos de óleos brutos. Terceiro Congresso Internacional da Sociedade Brasileira de Geofísica, Rio de Janeiro, 1993.

[46] GRANA, D.. Bayesian Inversion Methods for Seismic Reservoir Characterization and Time-Lapse Studies. PhD thesis, Stanford University, 2013.

[47] GJERDING, K.; SKJEI, N.; HAALAND, A. N.; MACHECLER, I. ; COLÉOU, T.. Geomodel update using 4-d petrophysical seismic inversion on the troll west field. In: 72ND EAGE CONFERENCE \& EXHIBITION INCORPORATING SPE EUROPEC 2010, 2010.

[48] GRANA, D.. Probabilistic approach to rock physics modeling. GEOPHYSICS, 79(2):D123-D143, 2014.

[49] HAN, D.-H.. Effects of porosity and clay content on acoustic properties of sandstones and unconsolidated sediments. PhD thesis, University of Stanford, 1986.

[50] HILL, R.. Elastic behavior of crystalline aggregate. In: PROCEEDING OF THE PHYSICAL SOCIETY, 1952.

[51] HASHIN, Z.; SHTRIKMAN, S.. A variational approach to the elastic behavior of multiphase materials. In: J. MECH. PHYS. SOLIDS, 11, p. $127-140,1963$.

[52] ISAAC, J. H.; LAWTON, D. C.. Avo analysis of carbonates. In: CREWES RESEARCH REPORT — VOLUME 21, 2009.

[53] JAKOBSEN, M.; JOHANSEN, T. A. ; MCCANN, C.. The acoustic signature of fluid flow in complex porous media. Journal of Applied Geophysics, p. 219-246, 2003. 
[54] JAKOBSEN, M.; HUDSON, J. A. ; JOHANSEN, T. A.. T-matrix approach to shale acoustics. Geophysical Journal International, 154(2):533558, 2003.

[55] KUSTER, G.; TOKSOZ, M.. Velocity and attenuation of seismic waves in two-phase media: Part i. theoretichal formulations. In: GEOPHYSICS, 39, p. 587-618, 1974.

[56] KUSTER, G.; TOKZOS, M.. Velocity and Attenuation of Seismic Waves in Two-Phase Media: Part II. Experimental Results, volumen 9. Geophysics, oct 1974.

[57] KURODA, M. C.; VIDAL, A. C. ; PAPA, J. P.. Analysis of porosity, stratigraphy, and structural delineation of a brazilian carbonate field by machine learning techniques: A case study. In: INTERPRETATION, VOL. 4, NO. 3, p. T347-T358, 2016.

[58] KITTRIDGE, M. G.. Investigating the influence of mineralogy and pore shape on the velocity of carbonate rocks: Insights from extant global data sets. In: INTERPRETATION, VOL. 3, NO. 1 - SEG AND AAPG, p. SA15-SA31, 2015.

[59] KUMAR, M.; HUA HAN, D.. Pore shape effect on elastic properties of carbonate rocks. In: SEG/HOUSTON 2005 ANNUAL MEETING, 2005.

[60] LIU, E.; PAYNE, M.; BAECHLE, G. ; HARRIS, C.. Carbonate rock physics issues. International Petroleum Technology Conference, 2009.

[61] DE A. LIMA NETO, I.; MISSAGIA, R. M.. Estimate of elastic properties including pore geometry effect on carbonates: A case study of glorieta-paddock reservoir at vacuum field, new mexico. In: REVISTA BRASILEIRA DE GEOF'ISICA (2012), p. 519-531, 2012.

[62] LUCIA, F. J.. Carbonate Reservoir Characterization. Springer, first edition, 2007.

[63] LI, Y.; GOODWAY, B. ; DOWNTON, J.. Recent advances in application of avo to carbonate reservoirs. In: CANADIAN SOCIETY OF EXPLORATION GEOPHYSICISTS - VOL. 28 NO. 03, 2003.

[64] METROPOlis, N.; ROSEnBLUTH, A. W.; ROSENBLUTH, M. N.; H., T. A. ; TELLER, E.. Equations of state calculations by fast computing machines. Journal of Chemical Physics, 21, 1953. 
[65] DE FIGUEIREDO, L. P.; GRANA, D.; SANTOS, M.; FIGUEIREDO, W.; ROISENBERG, M. ; NETO, G. S.. Bayesian seismic inversion based on rock-physics prior modeling for the joint estimation of acoustic impedance, porosity and lithofacies. Journal of Computational Geophysics, 336, 2017.

[66] GRANA, D.. Bayesian linearized rock-physics inversion. Geophysics, 81, 2016.

[67] MAHMOUD, S.; OTHMAN, A.; SOROKA, W.; ROMERO, T. ; SMITH, A.. A modeling feasibility study indicates seismic avo has potential to discriminate between brine and hydrocarbon in middle east carbonate reservoir. In: ABU DHABI INTERNATIONAL PETROLEUM EXHIBITION AND CONFERENCE, 2008.

[68] MAVKO, G.; T.MUKERJI ; J.DVORKIN. The Rock Physics Handbook: Tools for Seismic Analysis of Porous Media. Cambridge University Press, second edition edition, 2009.

[69] MICHOU, L.; LAFET, Y.; COLÉOU, T. ; PRZYBYSZ-JARNUT, J.. 4d seismic inversion on continuous land seismic reservoir monitoring of thermal eor. In: 75TH EAGE CONFERENCE \& EXHIBITION INCORPORATING SPE EUROPEC 2013, 2013.

[70] MACHECLER, I.; COLEOU, T.; GJERDING, K.; SKJEI, N.; HAALAND, A. N. ; RISTE, P.. 4D petrophysical seismic inversion on the Troll West field, p. 1780-1784. 2009.

[71] FERREIRA, T.; RASBAND, W.. Imagej user guide - ij 1.46r. Image J User Guide, 2012.

[72] OSTRANDER, W. J.. Plane-wave reflection coefficients for gas sands at non normal angles of incidence. In: GEOPHYSICS, 49, p. p. $1637-1648,1984$.

[73] BROOKS, S. P.. Markov chain monte carlo method and its application. J. Roy. Stat. Soc. D-Sta, 47, 1998.

[74] PAYNE, S. S.; WILD, P. ; LUBBE, R.. An integrated solution to rock physics modelling in fractured carbonate reservoirs. In: SEG DENVER 2010 ANNUAL MEETING, 2010.

[75] RAYMER, L. L.; HUNT, E. R. ; GARDNER, J. S.. An improved sonic transit time-to-porosity transform. 21st Annual Logging Symposium, 1980. 
[76] REUSS, A.. Berechnung der fliebgrenzen von mischkristallens auf grund der plastizitätsbedingung für einkristalle. Zeitschrift für Angewandte Mathematik und Mechanik, 9:49-58, 1929.

[77] COLEOU, T.; FORMENTO, J. L.; JENSEN, M. G.; VAN WIJNGAARDEN, A. J.; HAALAND, A. N. ; ONA, R.. Petrophysical seismic inversion applied to the troll field, p. 2107-2111. 2006.

[78] RUIZ, F.; DVORKIN, J.. Rock physics model for deep sea shallow calcareous sediment with porous grains, p. 1599-1603. 2007.

[79] RUSSELL, B.; HAMPSON, D.. The old and the new in seismic inversion. In: CANADIAN SOCIETY OF EXPLORATION GEOPHYSICISTS RECORDER, p. 5-11, 2006.

[80] RODRIGUES, L.; NUNES, J. ; GUÉRILLOT, D.. Evolution of seismic responses due to co2 injection in carbonates including chemical reactions and rock-physics model. In: ECMOR XIII - 13TH EUROPEAN CONFERENCE ON THE MATHEMATICS OF OIL RECOVERY, 2012.

[81] SAIN, R.; CHEN, G.; XU, S.; PAYNE, M. A. ; SULTAN, A. A.. Carbonate rock physics: Geophysical and petrophysical pore types of carbonate rocks from an offshore carbonate field, p. 1655-1659. 2008.

[82] SPIKES, K.. Probabilistic Seismic Inversion Based on RockPhysics Models for Reservor Characterization. PhD thesis, Stanford University, 2008.

[83] VOIGT, W.. Lehbuch der kristallphysik. Teubner-Verlag, 1910.

[84] VRUGT, J. A.. Markov chain monte carlo simulation using the dream software package: Theory, concepts, and matlab implementation. Environmental Modelling and Software, 75:273-316, 2016.

[85] KEYS, R.; XU, S.. An approximation for the xu-white velocity model. Geophysics, 67(5):1406-1414, 2002.

[86] XU, S.; PAYNE, M. A.. Modeling elastic properties in carbonate rocks. The Leading Edge, 28(1):66-74, 2009.

[87] XU, S.; WHITE. A new velocity model for clay-sand mixtures. Geophysical Prospecting, 1995. 
[88] WEGER, R. J.; BAECHLE, G. T.; MASAFERRO, J. L. ; EBERLI, G. P.. Effects of porestructure on sonic velocity in carbonates. SEG 74th Annual Meeting, 2004.

[89] WOOD, A. W.. A Textbook of Sound. The MacMillan, 1955.

[90] WANG, Z.. Seismic properties of carbonate rocks. In: CARBONATE SEISMOLOGY, VOLUME 6, p. 29-52, 1997.

[91] ZHAO, L.; NASSER, M. ; HUA HAN, D.. Quantitative geophysical pore types characterization and its geological implications in carbonate reservoirs. 2013.

[92] GRANA, D.. Probabilistic approach to rock physics modeling. Geophysics, Vol. 79:D123-D143, 2011.

[93] COSTA, M.; SILVA, E.; SANTOS, M. ; VASQUEZ, G.. Quantification of $4 \mathrm{~d}$ seismic response in pre-salt carbonates via time shift analysis. Third EAGE/SBGf Workshop 2016.

[94] VASQUEZ, G. F.. Ct irps 033/15. Petrobras Internal publication.

[95] BAECHLE, G. T.; COLPAERT, A.; EBERLI, G. P. ; WEGER, R. J.. Effects of microporosity on sonic velocity in carbonate rocks. p. 10121018, 2008. 


\section{A \\ Pores Aspect Ratio Measurements in the Thin Sections Images}

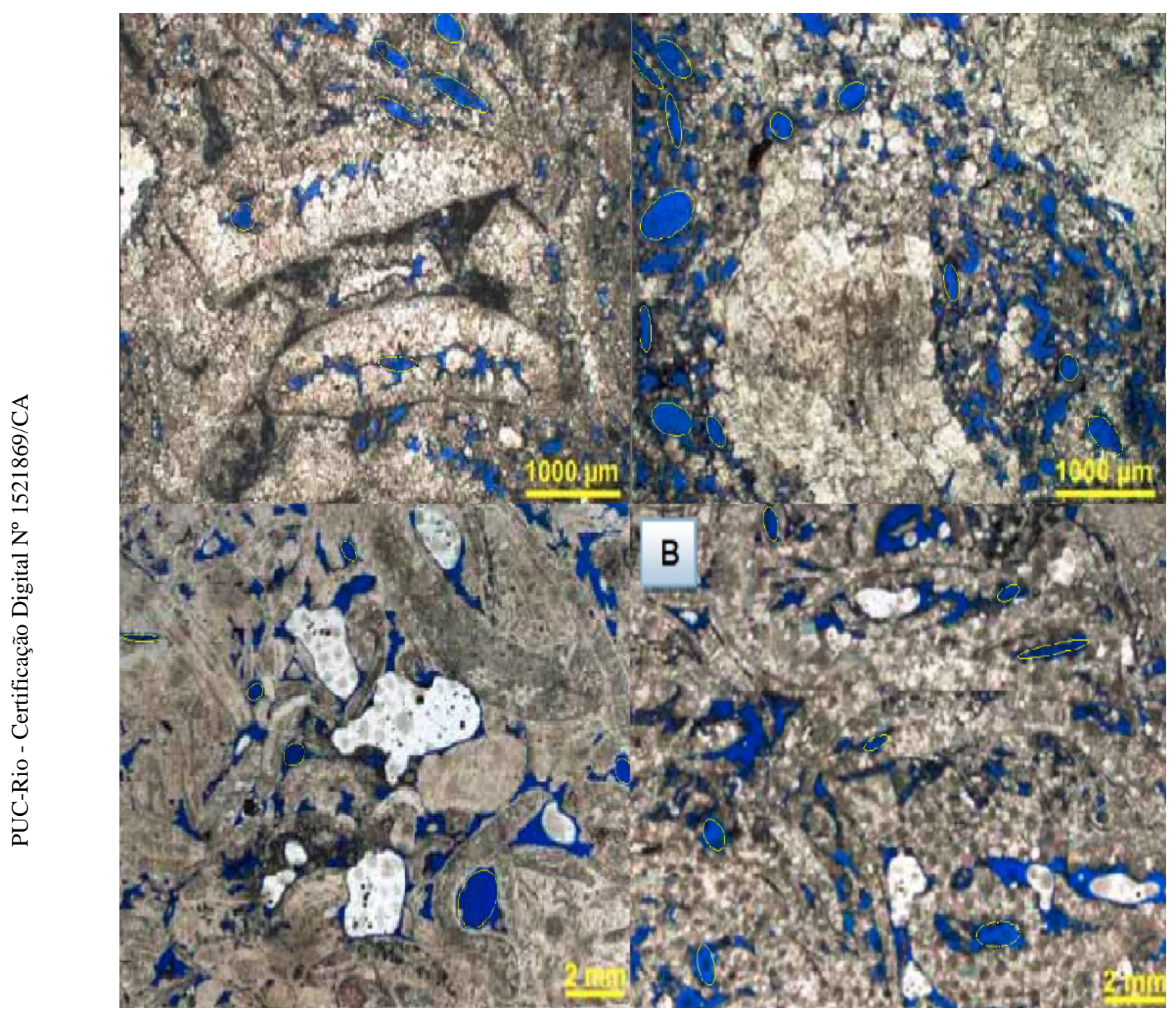

Figure A.1: Yellow ellipses fitted in the pores of the thin sections images through the ImageJ software. 
B

DREAM's outcomes - $\mathrm{Xu}$ and Payne and T-Matrix Rock Physics Models - Well 49

Table B.1: RMSE of the MAP values using both Rock Physics models in the DREAM algorithm - Well 49.

RPM RMSEMAP - Well 49

$\begin{array}{ll}\text { T-Matrix } & 133,12 \\ \text { Xu \& Payne } & 133,42\end{array}$

